\title{
Steering nanofibers: An integrative approach to bio-inspired fiber fabrication and assembly
}

\section{Citation}

Grinthal, A., S.H. Kang, A.K Epstein, M. Aizenberg, M. Khan, and J. Aizenberg. 2012. "Steering Nanofibers: An Integrative Approach to Bio-Inspired Fiber Fabrication and Assembly." Nano Today 7 (1) (February): 35-52. doi:10.1016/j.nantod.2011.12.005.

\section{Published Version}

doi:10.1016/j.nantod.2011.12.005

\section{Permanent link}

http://nrs.harvard.edu/urn-3:HUL.InstRepos:37255928

\section{Terms of Use}

This article was downloaded from Harvard University's DASH repository, and is made available under the terms and conditions applicable to Open Access Policy Articles, as set forth at http:// nrs.harvard.edu/urn-3:HUL.InstRepos:dash.current.terms-of-use\#OAP

\section{Share Your Story}

The Harvard community has made this article openly available.

Please share how this access benefits you. Submit a story.

\section{Accessibility}




\section{Highlights}

Integrative approach to creating fibrous surfaces with multiscale complexity and dynamic behavior.

Low-cost benchtop techniques to generate libraries of nanofiber arrays with fine-tuned parameters.

Program fiber arrays to self-assemble into complex, chiral, hierarchical architectures.

Encode reversible, responsive behavior through rationally chosen surface chemistry. 


\title{
Steering nanofibers: An integrative approach to bio-inspired fiber fabrication and assembly
}

\author{
A. Grinthal ${ }^{\mathrm{a}}$, S. H. Kang ${ }^{\mathrm{a}, \mathrm{b}}$, A. K Epstein ${ }^{\mathrm{a}, \mathrm{b}}$, M. Aizenberg ${ }^{\mathrm{b}}$, M. Khan ${ }^{\mathrm{b}}$, J. Aizenberg ${ }^{\mathrm{a}, \mathrm{b}, \mathrm{c}, \mathrm{d}, *}$ \\ ${ }^{a}$ School of Engineering and Applied Sciences, Harvard University, United States \\ ${ }^{\mathrm{b}}$ Wyss Institute for Biologically Inspired Engineering, United States \\ ${ }^{c}$ Department of Chemistry and Chemical Biology, Harvard University, United States \\ ${ }^{\mathrm{d}}$ Kavli Institute for Bionano Science and Technology, United States \\ * Corresponding author at 29 Oxford Street, Pierce 209, Cambridge, MA 02138, FAX: (617) \\ 496-1457, E-mail: jaiz@ seas.harvard.edu \\ Address: 29 Oxford Street, Cambridge, MA 02138, United States.
}

\begin{abstract}
As seen throughout the natural world, nanoscale fibers exhibit a unique combination of mechanical and surface properties that enable them to wind and bend around each other into an immense diversity of complex forms. In this review, we discuss how this versatility can be harnessed to transform a simple array of anchored nanofibers into a variety of complex, hierarchically organized dynamic functional surfaces. We describe a set of recently developed benchtop techniques that provide a straightforward way to generate libraries of fibrous surfaces with a wide range of finely tuned, nearly arbitrary geometric, mechanical, material, and surface characteristics starting from a single master array. These simple systematic controls can be used to program the fibers to bundle together, twist around each other into chiral swirls, and assemble into patterned arrays of complex hierarchical architectures. The delicate balance between fiber elasticity and surface adhesion plays a critical role in determining the shape, chirality, and higher order of the assembled structures, as does the dynamic evolution of the geometric, mechanical, and surface parameters throughout the assembly process. Hierarchical assembly can also be programmed to run backwards, enabling a wide range of reversible, responsive behaviors to be encoded through rationally chosen surface chemistry. These strategies provide a foundation for designing a vast assortment of functional surfaces with anti-fouling, adhesive, optical, water and ice repellent, memory storage, microfluidic, capture and release, and many more capabilities with the structural and dynamic sophistication of their biological counterparts.
\end{abstract}

\section{Highlights}

Integrative approach to creating fibrous surfaces with multiscale complexity and dynamic behavior.

Low-cost benchtop techniques to generate libraries of nanofiber arrays with fine-tuned parameters.

Program fiber arrays to self-assemble into complex, chiral, hierarchical architectures.

Encode reversible, responsive behavior through rationally chosen surface chemistry. 


\section{Keywords}

nanofibers, self-assembly, chirality, hierarchical architectures, surface chemistry, fibrous surface, nanofabrication, nanoscale mechanics, dynamic and responsive surface, bioinspired

\section{Introduction}

It's difficult to find a process in the natural world that doesn't rely on nano/microscale fibers aligning, bundling, or winding around each other. Plants strengthen themselves to stand upright by twisting cellulose fibrils into a spiral as they grow [1,2]; bones adapt to impact by helically intertwining collagen fibers around an axis normal to the force [3]. We hear when sound bends bundles of cilia [4], each of which is itself a bundle of sliding, reorienting microtubules [5], and we remember through stabilizing proteins winding around axons and axons bundling into nerve fibers. Beetles crawl upside-down by clustering and unclustering adhesive fibers on the bottom of their feet [6], and the genetic code copies itself through DNA strands winding into helices [7], coiling into chromatin fibers [8], and assembling into chromosomes. Nature's choice of fiber assembly as a modus operandi likely derives simply from a fiber's proportions: the high surface area to volume ratio provides both a multitude of ways to interact along its length and the corresponding mechanical flexibility to bend into many different configurations - particularly at the nanoscale where the combination of high aspect ratio and small radius makes fibers especially soft, with bending forces often on a par with surface attraction forces [9]. The resulting versatility makes it possible to create an assortment of dramatically different materials from the same starting fibers [10]: plants control the stiffness and toughness of wood by varying the helix angle of their cellulose fibrils [2], and the completely different mechanical properties of wood and bamboo have been attributed to differences in the number and orientation of microfibrils [1]. Since the initial product of fiber assembly is commonly a fiber itself, with a new compliance and surface profile, the process can repeat itself to produce a series of multiscale fiber bundles, each with unique properties and increasing hierarchical complexity. Moreover, the energy differences among the many possible configurations are often small, such that the assembled fibers can readily reconfigure dynamically.

One of the most interesting ways nature makes use of these fiber properties, and one that holds increasing allure as a nanotechnology design strategy, is to create complex functional surfaces from arrays of surface-anchored fibers. By aligning, assembling, and moving in a coordinated manner, anchored fibers generate a vast assortment of emergent surface behaviors. The water resistance of hairy plant leaves [11] and insect legs [12], the reversible adhesion of setae-covered gecko feet [13], the responsive temperature regulation of furry skin [14], and the sensing [15], self-cleaning, and swimming [16] of ciliated cells have generated intense interest in using this strategy to design functional surfaces with anti-fouling [17], microfluidic mixing [18, 19], particle trapping [9], drug delivery, dry adhesive [20], water-repellent [21-23], self-cleaning, optical [24, 25] and thermal [26] properties. All of these collective behaviors rely on the complex mechanical and surface characteristics of the individual fibers: directed beating patterns depend on bending asymmetry [27], and adhesion depends on a combination of fiber shape and compliance $[13,28]$. Yet fabricating synthetic fibers with nanoscale precision and complexity is 

natural systems, the capacity of nanoscale fibers to self-assemble into so many diverse composite patterns, with hierarchical control over compliance and surface properties, potentially offers a perfect opportunity to program an immense assortment not only of fibers but of tunable surface behaviors that arise from their coordinated large-scale interactions. At the same time, the surface-anchored configuration itself offers the potential for a unique level of control over the assembly process. The fact that the base of each fiber stays fixed can be used to introduce asymmetry into how fibers meet, deform, and associate, can generate long range-order, and provides a restoring force that makes each stage of assembly inherently reversible [9].

And yet, the complex, winding, dynamic structures that would seem to follow naturally from the marriage of surface-anchored mechanics with nanoscale fiber properties are almost completely absent from synthetic fibrous surfaces. Instead, under the name "lateral collapse", surface-based fiber assembly has historically been the losing end of a battle to fabricate stable fiber arrays in the first place. The tendency of fibers to bend and interact of course makes them prone to random, uncontrolled associations [29]. Although recent progress has produced design criteria for overcoming this problem [30-32], and interest in using nanofiber self-assembly as a surface fabrication step is increasing [33-36], the need to prevent chaos still weighs down most efforts to program specific structures. On the technical side, by the time fibers are made stiff, short, or non-adhesive enough to prevent spontaneous interaction, the possibilities for programming assembly are substantially reduced. Once assembly does occur, the desire to stabilize the new structure likewise complicates programming reversibility. Harnessing the selforganizing potential of anchored fibers is therefore a formidably tricky balance, particularly since the array design must also meet the requirements for specific downstream functions. On the conceptual side, the design principles that do exist for programmed assembly are largely inherited from the prevention studies. Since these tend to focus on finding the simplest route to stability, they consider only a few parameters of the fiber arrays, often in isolation. Within this technical and conceptual framework, several promising structures have been assembled [25, 3740], but much of the potential that nature reminds us is inherent to nanoscale fiber assembly has long gone unaccessed and has rarely even been looked for.

We have sought to cast a wider net and found that by simply taking a more comprehensive approach to programming the fibrous surface, we not only gain access to previously unthinkable structures but can begin to map out strategies for rationally designing them. Like much of the previous work, we use the capillary forces generated by an evaporating liquid to orchestrate motion of the fibers. As has been well studied, this method brings fibers together if the meniscus between them provides enough force to overcome their resistance to bending $[9,31]$. However, this apparent simplicity belies a sobering assortment of factors that collectively influence the net force felt by each fiber, how it responds, how fibers interact with each other once they meet, and what happens after the surface dries. To use this complexity to our advantage, we have explored a correspondingly wide assortment of surface parameters related to the mechanics, fiber geometry, surface chemistry, capillarity, and overall array configuration (Fig. 1). Surface chemistry has previously been considered as an all or nothing way to keep fibers apart or glue them together, but never as a more subtle way to control assembly size, shape, or dynamics. The 2D lattice of the array has been painstakingly pre-patterned [39], but simple 3D variations such as fiber orientation have likewise not been considered as programming elements. Similarly, anisotropy in fiber geometry [36, 40] and material composition has received limited attention despite its widespread use in nature. The role of 

[41]. Beyond programming individual surface features, we exploit the subtle interplay between different parameters to develop an integrated, collective strategy for controlling assembly of nanofibers.

Programming all of these features systematically and combinatorially would be nearly impossible if we had to fabricate a new master array from scratch every time we adjusted a parameter. In this review, we first discuss the set of simple benchtop techniques we have developed for straightforwardly generating libraries of fibrous surfaces from a single master surface. These methods provide an indispensable foundation for all of our subsequent work by allowing us to create arrays of nanofibers that can respond to force and assemble in arbitrary, highly programmable ways. We then discuss the fascinating collection of self-assembled structures that can be generated from these simple but finely tuned surfaces, and provide programming criteria for creating specific shapes with controlled hierarchical organization. Based on the principles derived from this work, we present a strategy for endowing the assembled structures with a wide range of dynamic, responsive behaviors through rationally chosen surface chemistry.

\section{Fabrication platform and parameters: From $A$ to $Z$ a. Benchtop soft lithographic double-casting method}

Over the last several years, we have developed a comprehensive materials-based platform for the fabrication of bio-inspired surfaces and nanofibers whose assembly can be finely controlled. In contrast to conventional fabrication techniques such as photolithography and electron- beam lithography, our platform does not require use of a clean room and is easily performed on the benchtop. Many degrees of freedom-material, geometric, mechanical, dynamic - can be programmed starting from a single, simple master structure. And whereas conventional methods to produce regular arrays of surface-attached nanofibers are restricted to a very limited choice of materials and a highly sophisticated and expensive fabrication process, our approach enables application of a wide range of materials, including metals, polymers, and ceramics.

At its core, our fabrication platform adopts and significantly extends the soft lithography method originally pioneered by Whitesides, et al. [42] Soft lithography is extremely effective for faithful replication of microfabricated substrates in an elastomeric polymer such as polydimethylsiloxane (PDMS). However, fabricating high-aspect-ratio (HAR) fibers inspired by biological structures such as neuromasts or cilia requires more stability and rigidity than provided by such a soft elastomer. In our approach, therefore, we employ a double-replication approach, and we cast the final replica in the material or materials of choice. With this basic approach, it becomes possible to tailor the properties of HAR structures' stability, stiffness, and actuation through material design, to modify the symmetry of the nanostructure array through specific deformations of the elastomeric mold during casting, and to effect nontrivial transformations of the nanostructure geometry and surface chemistry following the casting step [43-46].

In the overall fabrication platform (Fig. 2), the initial master surface can be formed by standard lithographical techniques, grown bottom-up (for example, nanowires), or even be a live biological sample [47]. Nearly any topography can serve as a master surface, with the main 
requirement being that the negative mold must be able to peel off or detach easily, ensuring that the master remains undamaged and is faithfully replicated. The master can also be treated with a nonstick surface chemistry such as fluorinated silane to aid peel-off. From a fabrication design perspective, parameters such as the fiber spacing, radius, length, etc., can be specified in the master, but these parameters can also be extensively modified downstream in our platform. In Fig. 2A, we have taken a Si nanofiber array that was produced by Bosch etching, a pulsed etching-passivation technique that allows very high aspect ratio structures to be made [48]. The pitch (center to center distance between the posts) is $a_{0}=2 \mu \mathrm{m}$, post radius $r_{0}=125 \mathrm{~nm}$ and post length $l_{0}=8 \mu \mathrm{m}$, for an aspect ratio of 32 .

We cast a negative replica of the fibrous master in either PDMS or other nonstick materials, such as polyfluoropolyether (PFPE) and paraffin (Fig. 2B-E). PDMS offers a number of advantages: it is inert, reusable, and inexpensive. It is compliant and elastically deforms up to $300 \%$ strain, such that it releases well even from rough master topographies. A PDMS mold can even be used to cast PDMS replicas by applying a fluorinated layer to the mold surface. In any case, the mold (Fig. 2D-E) has an array of wells, into which the final replica material is cast in liquid or vapor form and solidified (Fig. 2F). The mold is then either peeled off (PDMS, PFPE) (Fig. 2G) or heated and sacrificially dissolved (paraffin). Figure $2 \mathrm{H}$ shows an epoxy replica of the original silicon HAR nanofiber array, which is indistinguishable by SEM.

\section{b. Breaking the one-to-one replication barrier with elastomeric mold deformation: array-level geometry control}

The fabrication of fibrous surfaces with varying geometric parameters using a basic oneto-one double-replication procedure can become prohibitively expensive, requiring customized Si masters for each new design. Moreover, conventional lithography limits fabrication to normally oriented nanofibers. In biological systems, however, natural high-aspect-ratio fibers are often oriented in various directions and have a range of anisotropic cross-sections with important functional implications [49, 50]. For example, tilted fibers are critical to the adhesive properties of gecko feet, while the elliptical cross-section of superficial neuromasts - structures that detect water flow on the surface of fish - allows them to discern the direction of fluid flow $[51,52]$.

One breakthrough of our approach is that the replication no longer need be a one-to-one process. Nontrivial geometric transformations from the master to the replica are easily achieved by deforming the flexible negative PDMS mold after casting but before solidifying the final replica material. The mold deformation technique allows us to form a wide range of nanofiber array geometries, including different 2D lattice symmetries and fiber cross-sectional shapes, all based on the same original master [43]. We can uniaxially extend, compress, bend, shear, or twist the mold shown in Fig. 2D to transform the original geometry, as shown in Figure 2I-P. PDMS is an optimal mold material in this regard because its low Young's modulus and high elastic limit differentiate it from rigid molds that strictly permit one-to-one replication.

There are several types of array-level geometric control enabled by PDMS mold deformation, and the range of possible outputs is summarized in Table 1. For example, by applying extension or compression to the negative mold along any direction in the plane of the substrate, we are able to transform an originally square lattice symmetry to rectangular or rhombic, and an originally circular fiber cross-section to elliptical (Figure 2I-J). The deformation magnitude and direction simultaneously determine the final unit cell of the 

transformation process, and there is a coupling of feature and array-level geometry control. Tilted structures with a desired orientation are formed by applying shear to the mold, as shown in Fig. 2M. The magnitude and direction of the shear directly determine the resulting nanofiber tilt angle and direction. The length of the fibers, $l_{0}$, can be changed by compressing the negative mold perpendicular to the 2D array (Fig. 2N), and macroscopically curved nanofiber substrates (Fig. 2L,P) are easily designed to match an arbitrary contour. Most importantly, any combination of deformation types can be applied either in parallel or in series (via multiple double-replication cycles) for sophisticated geometric control. Figure 2Q shows a top view of an example of an epoxy nanostructured surface fabricated using a compound shear and $45^{\circ}$ extension of the mold.

\section{c. Control of the mechanical and other properties by material selection}

Another key advantage of our fabrication platform is the ability to specify the materials comprising the fabricated nanofibers. Whereas conventional lithographic procedures are limited essentially to silicon, the Young's modulus of a double-replicated nanostructure can vary from less than one MPa to hundreds of $\mathrm{GPa}[43,53]$. Using this method one can form replicated nanostructures from synthetic as well as biological polymers (e.g., epoxy, hydrogels, silk, shape memory polymers), metals with a low melting point (e.g., Wood's and Field's alloys), and ceramics deposited through atomic layer deposition (e.g., alumina), as shown in Fig. 2 R. Furthermore, the Young's modulus of the array can be finely controlled by varying the ratio of two different prepolymers. Indeed, by variably mixing two epoxy formulations, we have produced epoxy nanofiber surfaces with a modulus ranging from tens of $\mathrm{MPa}$ to several $\mathrm{GPa}$ [43]. The modulus range of such a two-polymer system was extended another order of magnitude through compositing silica nanoparticles in the polymer matrix, reaching tens of GPa. A modulus-versus-composition calibration curve based on a series of material tests provides us the means to control fiber modulus and functions such as assembly [9].

In addition to using ceramic-polymer composites for programming the mechanical stiffness of a nanofiber, we can also introduce magnetic responsiveness by incorporating magnetic particles in the fibers. These particles can be drawn to the tips of the fibers using a magnet prior to polymer curing, such that applied forces will lead to efficient bending.

Beyond monolithic replicas comprised of a uniform material system and mechanical properties, we have extended our method to make possible spatially defined multi-material nanofibers for sophisticated sensitivity and assembly behavior (Fig. 2T-W). To achieve this, multiple casting materials are stratified during the casting process depicted in Fig. $2 \mathrm{~F}$ to vary relevant properties along the height of the structure. The fibers may be fabricated from a stiff replica material (shown in green), for example, while the bulk substrate is flexible (shown in red); or there can be a compliant interlayer linking the stiff nanostructures and the stiff substrate. To fabricate a multi-material nanoarray, additional steps are added to the core fabrication process. Once the replica prepolymer is applied to the negative mold and allowed to fully penetrate the wells, the excess is removed. At this point, a compliant interlayer can be spincoated on at a lower speed and cured, followed by regular casting of the substrate [53]. Material property gradient control between the bulk and nanofiber materials is possible, for example by varying the degree of crosslinking of one material layer before interdiffusion with the next layer.

Such a "flexible anchoring" configuration, depicted in Fig. 2W, is of great functional interest. Biological structures such as the superficial neuromasts of fish depend on stiffness 
gradients and anisotropy [54], pointing to important applications in the fabrication of engineered nanoarrays for flow sensors and sophisticated artificial cilia. The higher elastic range of motion allowed by flexible anchoring points also suggests that these nanostructures are mechanically more resilient than their monolithic analogues.

In addition to spatial control over material composition, we can also vary the surface chemistry of the fibers (Fig. 2X) by using plasma treatment or functionalization. These treatments allow us to modulate the mechanical and adhesion properties independently and extend our control over surface-related phenomena such as wetting.

\section{d. Beyond proportional transformation: feature-level control of geometry, mechanics, and function}

Up to this point, our fabrication platform has augmented the core double-replication process with both extensive material and array-level geometry control. Yet programming mechanics, geometry, and function at the feature level, independent of the array-level properties, has not been possible. For example, deformation of a mold in the [hk0] direction intended to change nanoarray symmetry also changes the fiber cross-section, which may or may not be desired.

Recently we have developed a new nanofabrication method that allows an array of nanofibers to be precisely re-shaped by the deposition of conductive polymers. This method, referred to as "Structural Transformation by Electrodeposition on Patterned Substrates" (STEPS), breaks the proportional transformation barrier and makes it elementary to change feature-level dimensions and to produce nontrivial 3D transformations - all without changing the 2D lattice. The transformed nanofibers can then be used as masters for double-replication into the desired materials. Tapered, anisotropic, curved, and overhanging shapes can all be readily made by STEPS, and isolated nanostructures can even be bridged to form continuous networked arrays for new classes of bioinspired applications [45].

To produce feature-level geometric transformations, a metal layer (e.g. gold or platinum) is first deposited onto a HAR nanofiber array. Since our original Si nanofiber master is fabricated by the Bosch process, an iterative etching process, both the Si and replicated epoxy fiber walls are scalloped rather than smooth (which affects the metal layer and polymer deposition as discussed below.) The metal layer functions as an electrode, and when the sample is placed into a conductive monomer aqueous solution and voltage is applied, conductive polymer (e.g., polypyrrole) starts to electrochemically deposit on the metal. The rate of deposition is controlled by changing the voltage, while the size and shape of the resultant HAR fibers are precisely controlled by adjusting both the voltage and total electrodeposition time [45].

Figure $2 \mathrm{Y}$ summarizes three process schemes for controlling the feature-level geometric control of scalloped fibers. Depending on the metallization method, each scheme enables specific transformation types. In scheme I, a continuous electrode is formed by isotropic sputtering. Electrodeposition on sputter-coated electrodes (type I) results in a smooth, conformal film that increases the fiber dimensions by the deposited thickness in all directions. This effectively increases diameter without significantly changing length. At the same time, the spacing between adjacent pillars decreases and the space-filling factor of the substrate increases, as shown in Fig. $2 Z$ for a square array of HAR nanofibers. In STEPS types II and III, a set of discontinuous electrodes is formed by line-of-sight electron beam evaporation on the tops of 

nanofiber profile along the axial direction; hence, what is typically seen as a fabrication defect of the Bosch process is exploited in STEPS [55]. The large continuous electrode on the substrate surface causes polymer to deposit only there initially, but this layer eventually connects to the ring electrode on the first scallop and bridges the gap. From then on, the polymer continues jumping the "rungs" of an electrode "ladder" and the resulting gradient of deposition time from basal to distal ends of the nanostructure leads to the conical transformation as shown in Fig. 2Y,II. This enables applications such as mechanical reinforcement of nanoarrays for robustness [45], controlling fiber assembly-based particle capture properties, etc. New anisotropic geometries furthermore become possible when metal electrodes are deposited onto the nanostructures at an angle, forming C-shaped electrodes on each fiber scallop (Fig. 2Y,III). Electrodeposition on this substrate forms a polymer backbone on one side of each fiber, causing it to bend like a bimetallic strip. Such a curved geometry is distinct from and complements the straight tilted geometry produced by mold shearing. These curved nanostructures allow further control of anisotropy in assembly.

Perhaps most importantly, with the capability to create either continuous or stepwise gradient patterns from a single master structure, the final nanofiber arrays no longer need to be uniform over their entire area. We can perform either continuous or stepwise withdrawal of the substrate from the electrodeposition bath, leading to a gradient of electrodeposition time along the substrate withdrawal direction and therefore to a gradient of geometrical parameters. Multiple gradients, such as orthogonal or triaxial, can even be fabricated on a single substrate by rotating the axes of gradients over two or more electrodeposition steps. The resulting combinatorial libraries have made it possible for the first time to systematically study the combinatorial effects of geometric parameters on surface properties on a single substrate. This capability allows us to co-optimize the surfaces for self-assembly and a wide range of downstream applications, including nanotopographic cues for bacterial and cell behavior on patterned substrates, screening mechanical robustness of different taper profiles against abrasion, memory and storage, ciliary transport in microfluidics, fluid flow sensing, dry adhesion, and water and ice repellency [24, 56-58].

\section{e. Unifying the fabrication platform to program nanostructure performance}

The mechanics of nanofibers is a key issue when designing surfaces for functional assembly processes and applications in actuation/sensing. To control the mechanical sensitivity of the nanoarray, we may systematically apply the different parts of our fabrication platform, combining mold deformation, material selection, and STEPS feature-level transformation. Considering the final nanostructure, when a capillary or other stimulus force $F$ acts on the tip of a nanofiber of length $l$, perpendicular to the fiber, the tip deflection $\delta$ is given by $\delta=F l^{3} / 3 E I$, where $E$ is the Young's modulus and $I$ is the moment of inertia [59]. For a post with a circular cross-section of radius $r$, the moment of inertia is given by $I=\pi r^{4} / 4$. Given typical values for epoxy nanofibers of $E=1 \mathrm{GPa}, l=8 \mu \mathrm{m}, r=125 \mathrm{~nm}$, a force of about $1.1 \mathrm{nN}$ is needed to deflect the tip of the post by $1 \mu \mathrm{m}$. To control fiber flexibility we can adjust the radius (which scales as fourth power), the length (cubic power), the modulus (linear dependence), or any combination thereof [43]. As both geometric and material parameters control the nanoarray sensitivity, we consider the "effective stiffness" $S_{\text {effect }}=F / \delta$, where $\delta$ is the deflection, to help determine what strategy to employ for a target flexibility range. For nanofibers of arbitrary 
dimensions and materials, the ratio of their $S_{\text {effect }}$ allows their direct comparison, e.g., for a circular cross-section:

$$
\frac{S_{1 e} f}{S_{2 e} f}=\left(\frac{E_{e_{1}}}{E_{e_{2}}}\right)\left(\frac{l_{2 t}}{l_{1 t}}\right)^{3}\left(\frac{r_{1}}{r_{2}}\right)^{4}
$$

Complex 3D microstructures with tightly tuned mechanics can be fabricated simply by combining multiple STEPS methods, e.g., starting with a modified-feature nanoarray or its replica, and evaporating a new set of metal electrodes and performing subsequent STEPS procedures. Moreover, serial composition of STEPS can be combined through iterative moldcast cycles with the mold deformation and materials selection aspects of the overall fabrication platform for virtually limitless programming possibilities. Indeed, all these sub-methods can be combined over one or multiple fabrication cycles to yield an enormous range of mechanical, geometric, and material properties.

\section{Empirical fiber mechanical properties to validate design and evaluate for applications}

\section{a. Motivation for an empirical characterization method}

Designing functional nanoarrays with specific mechanical properties is only possible if we are able to characterize their real behavior and calibrate our predictions with empirical results. Indeed, we have up to this point relied on Eulerian beam mechanics to predict and design the sensitivity of a HAR nanostructure to the applied force. Given that these structures are in the submicron range, where surface area to volume ratios are significantly higher than in the macroscopic regime, it is not necessarily a given that macroscale beam mechanics can be applied without correction. Furthermore, many mechanical properties of nanoarrays cannot easily be modeled a priori because of their complexity and nonlinearity.

Relatively scant attention has been devoted to the mechanical robustness and fitness for technological application of nanofibrous surfaces beyond the bench. Along the lines of our previous nanoindentation study on a gradient of micropost taper profiles [45], the studies that have been reported principally rely on ensemble measurements [60-63]. Others are even coarser, applying such test methods as bulk abrasion [64]. Systematic mechanical tests of individual HAR surface nanofibers have not been described to our knowledge.

\section{b. Atomic force microscopy (AFM) for single-structure measurements of engineered polymeric nanostructures}

Taking a cue from atomic force microscopy (AFM) measurements of nanowire bridges and cantilevers [65-67], we have applied AFM to perform the single-structure mechanical measurements of our engineered fibers. We have developed a sample preparation method in which the test fibers are oriented horizontally so that the AFM cantilever can accurately apply zdirection bending forces. Spring constant calibration was performed for each cantilever used, allowing the mechanical properties of an individual fiber to be probed. Specific test locations were programmed along with open or closed loop test parameters.

To validate AFM-based single fiber measurement, we first tested the bending stiffness of HAR silicon fibers $\left(\mathrm{h}=6.8 \mu \mathrm{m}, \mathrm{r}_{\text {base }}=150 \mathrm{~nm}, \mathrm{r}_{\text {tip }}=90 \mathrm{~nm}\right)$, shown in Fig. 3A. By testing silicon, a very well characterized material, we sought to compare the empirical and analytical bending behavior. As noted above, Euler beam mechanics predicts an inverse cubic relationship 
between the bending stiffness of a cantilever and the length at which a force is applied (Fig. 3B, inset). The empirical stiffness-length curves are plotted in Figure 3b, yielding a power relationship of $-3.06 \pm 0.2$, highly consistent with the analytical value. In addition, the measured effective stiffness of the silicon nanofibers was consistent with its geometry from SEM images and its elastic constants. Thus the AFM characterization method has been shown to be viable and accurate, opening new avenues to measure the dynamic and nonlinear mechanical properties of bioinspired nanofibers.

\section{c. Application-critical dynamic mechanical properties: strain hardening, fatigue, viscoelasticity}

Unlike effective stiffness, a number of application-critical aspects of our polymer nanoarrays are difficult to predict a priori. Fatigue life and strain hardening at the micro/nanoscale, for example, should be measured empirically [68, 69], and these have critical bearing on application fitness. For example, synthetic cilia and neuromasts must flex constantly in performing their function, but their sensitivity would change with time in the case of straininduced stiffening or accumulation of fatigue damage. The performance characteristics would therefore drift over the operating lifetime of the nanoarray, which itself is expected to be finite.

We cyclically deflected and relaxed a square epoxy microfiber $\left(1=9.0 \mu \mathrm{m}, \mathrm{r}_{\text {base }}=1.05\right.$ $\mu \mathrm{m}, \mathrm{r}_{\text {tip }}=0.85 \mu \mathrm{m}$ ) by AFM tip and tracked its effective stiffness evolution, shown in Fig. 3C. A maximum tip displacement of $0.8 \mu \mathrm{m}$ from the initial position was maintained in each load cycle. The fiber was found to increase monotonically in effective stiffness from $10 \mathrm{~N} / \mathrm{m}$ by $30.6 \%$ over 15,000 load cycles (applied at $16 \mathrm{~Hz}$ and $91 \mu \mathrm{m} / \mathrm{s}$ ), consistent with strain hardening behavior. After this point the stiffness rapidly dropped off, indicating structural failure. From our empirical characterization it is apparent that (1) the force sensitivity of epoxy HAR $\mu$-structures will decrease over time; (2) alternatively, more energy will be needed to sustain a given displacement; and (3) epoxy nano/microfibers are subject to fatigue failure after an order of 10,000 cycles at $16 \mathrm{~Hz}$. These empirical properties are critical to consider in suitability assessment for different applications.

An additional nanofiber response property that is difficult to predict is viscoelasticity. A large relaxation time and hysteresis in the load-unload cycle would limit the frequency of stimuli that can be detected in sensor applications, for example, and high-speed assembly or actuation would require more energy. Applying our AFM characterization to a slightly thicker epoxy nanofiber that measured $17.22 \mathrm{~N} / \mathrm{m}$, we were able to characterize its load-unload behavior (Fig. 3D). The area highlighted in green depicts the hysteresis loop for a force curve of $20 \mu \mathrm{N}$ ( $\max$ deflection $=1.5 \mu \mathrm{m}$, rate of deflection $=6 \mu \mathrm{m} / \mathrm{s}$ ). This hysteresis of $32 \%$ of work done reveals that epoxy nanoposts are still primarily elastic over this bending range. At higher load frequencies, however, the hysteresis will of course increase [58] and should be considered in the application context.

\section{Programming self-assembly}

Self-assembly of nanoscale fibers has been reported in a variety of synthetic systems such as soft lithographic stamps [29], semiconductor and metal nanowires [70], carbon nanotubes [40, 71], and polymer microstructures mimicking the foot of a gecko [20]. Yet few approach the level of control over assembly size and shape seen in biological systems such as the beetle 
Hemisphaerota cyanea, which precisely modulates the assembly and disassembly of its tarsi to control its foot adhesion to a surface [6]. The programming capabilities made possible by the fabrication techniques described above have now enabled us to begin to develop an integrated understanding of the factors involved in controlling assembly and to create a wide assortment of nontrivial structures from simple nanofiber arrays.

In recent years, several studies have modeled capillary-induced self-assembly in terms of the competition between capillary force and the opposing elastic force [33, 38, 72]. Each analysis focused on a particular system and parameters, but in terms of the scaling relation, the derived equations can be summarized as $N \sim \frac{E_{c}}{E_{E}} \sim \frac{D^{2} \gamma \cos ^{2} \theta}{D^{4}(p-D)^{2} E / h^{3}} \sim \frac{\gamma h^{3} \cos ^{2} \theta}{D^{2}(p-D)^{2} E}$ (1), where $N$ is the average number of fibers in one assembly, $E_{\mathrm{C}}$ is the capillary interaction energy, $E_{\mathrm{E}}$ is the elastic energy term, $h$ is the height of the fibers, $\gamma$ is the surface tension of the liquid, $D$ is the diameter of the fiber, $p$ is the distance between the fibers, $E$ is the Young's modulus of the fiber, and $\theta$ is the equilibrium contact angle of the liquid on the surface of the fibers. While this relation was sufficient to describe assembly under the specific conditions investigated, it nonetheless lacks several general features. Assembly is predicted strictly by minimizing the energy of the final assembled structure, without taking into account the kinetics of the process [9, 73]. In addition, the direct fiber-to-fiber adhesion force that holds the final structures together after the liquid dries is not included as a factor. Finally, the relation considers only the number of fibers in the final structure and provides no insight into the evolution of the final assembly pattern and how complex assemblies and geometries such as chiral structures and hierarchical patterns might be formed.

To develop a more comprehensive understanding, we have used fiber arrays with systematically controlled properties as a model system. The typical array configuration was a square lattice with spacing ranging from 0.9 to $2 \mu \mathrm{m}$, and the fibers generally had a circular cross-section, a diameter between 250 and $500 \mathrm{~nm}$, and a height between 5 and $8 \mu \mathrm{m}$, comparable to the length scale seen in many biological systems $[12,13]$. Fibers were assembled by immersing the surface in a wetting liquid, generally ethanol, and letting the liquid evaporate at ambient temperature.

Even at the elementary level of four-fiber clusters, this system produces an assortment of unique shapes. In some cases the fibers form an arched structure, with the fibers touching only at their tips (Fig. 4A, left). While this configuration exhibits four-fold symmetry consistent with the square lattice, in other cases the tips slip such that the fibers cross instead of meeting head-tohead, and the fibers wind around each other to form a chiral structure (Fig. 4A, center). In still other cases, the fibers likewise interact beyond the tips over an extensive contact area, but they remain connected at the tips and simply zip together along their length to produce an achiral bundle (Fig. 4A, right). A self-assembled chiral structure has not been reported in any other mesoscale system composed of an array of surface-attached fibers, so it is particularly striking that it arises simply by varying basic geometric and surface parameters, without any initial chirality in the building blocks or environment as required in other systems [74] [75, 76]. We find that chirality emerges specifically within a limited window of diameter, elastic modulus, and surface adhesion strength. A high stiffness, resulting from either a large diameter or a high elastic modulus, appears to prevent chirality by disfavoring the extra bending required for fibers to twist around each other. In addition, strong surface adhesion prevents the tips from slipping from their initial contact point, leading instead to formation of a zipped structure. However, since the extra bending associated with formation of wound (or zipped) structures requires a stronger 
interaction force to counteract the elastic restoring force, the adhesion must be strong enough to hold the structure together. Controlling shape and chirality therefore relies on modulating the delicate balance between elastic and adhesion forces [77].

These various shapes can then serve as building blocks for creating complex higher order structures. For example, chiral bundles interact with each other to form larger-scale chiral architectures through a series of hierarchical steps (Fig. 4B-C). After an array of first order clusters forms (Fig. 4B-C, II), these clusters can combine to form second order assemblies (Fig. 4B-C, III). This process continues through formation of third, fourth, and higher order structures, with complexity increasing at each stage, as long as the driving force remains strong enough to counteract the elastic restoring force [78] (Fig. 4B-C, IV)). The kinetics of the assembly shows stepwise growth of the assembly size, such that the array proceeds through each hierarchical stage in unison (Fig. 4D).

As with the shape of the assembled bundles, controlling the number of hierarchical stages also relies on balancing the mechanical and surface properties of the system. Consistent with the sizes predicted by the previous models, the size of the assembly is inversely proportional to the diameter of the fibers (Fig. 5A). A similar trend is observed for increasing elastic modulus. We noted, however, that for the largest diameter fibers, the final size of the assembled bundle is always lower than predicted. Following the assembly process in real time showed that this anomaly results from partial disassembly: consistent with the predictions, larger assemblies initially form in the wet system when capillary force brings them together, but they dissociate as the system dries, presumably because the adhesion force is not strong enough to hold them together [77]. Although adhesion has received little attention compared to elastic and capillary force terms, the surface adhesion between fibers clearly plays a critical role in determining the final size of the assembly.

We directly assessed the influence of surface adhesion on hierarchical assembly by systematically modifying the surface chemistry of the fibers by plasma treatment. Though we do not have a quantitative value, the adhesion increases with increasing plasma treatment time. The trend we observe is completely opposite from what might be expected based on the disassembly discussed above: the cluster size decreases as adhesion strength increases (Fig. 5B). Although plasma treatment slightly reduces the fiber diameter by etching, this effect would also be expected to produce larger assemblies with increasing treatment time since it decreases the effective stiffness. This unexpected trend can be understood by considering the hierarchically evolving shape changes that accompany increasing adhesion. As discussed above, strong surface adhesion produces clusters that are zipped together along their length (Fig. 4A, right). This structure may be more difficult to bend, possibly analogous to the collaborative stiffening effect described for macroscopic systems [79].

Introducing anisotropy into the system provides a way to achieve even finer control over size, shape, and chirality. Anisotropic fibrous surfaces are commonly observed in biological systems, such as on the feet of water striders and geckos [12, 50]. By deforming the elastomeric mold during surface fabrication as described above, we have introduced various types of anisotropy into the fiber geometry and the configuration of the array. Stretching the mold to produce fibers with an elliptical cross-section and a rectangular lattice leads to anisotropic higher order clusters as shown in Fig. 5C. The differences in cluster size along the directions corresponding to the long and the short axes are consistent with the differences in elastic force terms expected from the corresponding geometries [77]. If we instead shear the mold, we generate tilted fibers, thus changing the orientation over the whole array while maintaining the 
original 2D square lattice. This approach produces different effects depending on the direction of the tilt. For example, while an array with normally oriented fibers forms four-fiber clusters (Fig. 5D), the same array with fibers tilted along a lattice direction forms exclusively two-fiber clusters (Fig. 5E). If the fibers are tilted slightly off the lattice direction, two-fiber clusters again form, but rather than random chirality the clusters now have a uniform handedness (Fig. 5F). Combined with balancing the overall elastic, capillary, and adhesion forces, simple small variations in the array can thus be used not only to introduce chirality into the system but to establish the handedness.

While these parameters provide a means for programming the shape and size of each assembly, controlling the long-range order of the system presents an additional challenge. For a square lattice, multiple domains tend to form due to random nucleation and propagation of clusters as shown in Fig. 6A. The system parameters contributing to long-range order were studied numerically [80] by modeling assembly as an irreversible sequential adsorption process on a square lattice. When four-fiber assemblies form on an initially empty lattice, they locally increase the probability of forming nearby clusters, thus generating ordered domains on a local scale. During this process, addition of adjacent fibers to the initial cluster is suppressed, analogously to excluded volume interactions for hard squares, due to the fact that as a fiber initially bends toward one neighbor to form a cluster, the neighbor on the other side experiences a weaker capillary force in that direction and tends to bend away from the forming cluster. However, two choices remain for the location of neighboring clusters. If they form directly next to the original cluster, the long-range order is preserved, but if they form at a diagonal position the order is disrupted and multiple domains are created. Using two parameters, we explained how these parameters control the likelihood of cluster formation at adjacent and diagonal positions.

This study suggested that controlling the evaporation process might promote long-range order by preventing assembly from propagating in a random manner. Consistent with this idea, we have shown that controlling meniscus movement allows us to form a large area with a single domain (Fig. 6B) [81]. The evaporation process can be further manipulated to control the longrange pattern of the assembly. By placing a patterned mask over the array, we can induce bending of the posts along preferential directions such that the overall assembly forms longrange patterns along the boundaries of the patterned regions of the mask (Fig. 6C). Moreover, if the periodicity of the mask pattern approaches that of the nanofiber array, we can induce deterministic bending of the fibers to form an ordered pattern on the scale of the substrate[82].

For more precise control at the local level, we can introduce artificial nuclei at specific locations by assembling fibers using an electron beam [83] before introducing a liquid. By controlling the spacing of artificial nuclei, we can enhance long-range order. Electron beaminduced assembly can also be used to create surface-specific patterns by localizing the electron beam at desired locations (Fig. 6D).

\section{Encoding dynamic and reversible self-assembly with surface chemistry}

Beyond creating arrays of static structures, the function of self-assembled systems often requires structural stability to be balanced with the capacity to undergo dynamic cycles of assembly and disassembly. The results from our group described above $[9,77]$ and those from others [33] point to the importance of fiber surface adhesion in determining the stability of 

encoding reversibility by systematically varying the surface chemistry of the fibers [84], allowing us to program our assembled structures for dynamic, responsive behavior mimicking that of many biological systems.

As a model system for calibrating the influence of different surface chemistries, we used a simple fiber array whose geometric and mechanical properties were chosen to yield clusters of at most four fibers. Structures satisfying this condition consisted of microfibers $1.5 \mu \mathrm{m}$ in diameter and $10 \mu \mathrm{m}$ in height, with a pitch of $8 \mu \mathrm{m}$, and made of epoxy resin UVO114. We modified the surface chemistry by coating the fibers with a thin gold layer and functionalizing with self-assembled monolayers (SAMs) of organic molecules attached via a thiol group. The terminal functional groups of the SAMs were chosen to provide a spectrum of intermolecular bond strengths, from the weak van der Waals bonds for methyl-terminated SAMs, to the range of hydrogen bonds for $\mathrm{NH}_{2}-\mathrm{OH}-$, and $\mathrm{COOH}$-terminated SAMs, to the strong covalent bonds in the case of SH-end groups. For finer tuning, we also varied the carbon chain length for each functional group. Specifically, we used the following surface molecules: Cysteamine $\left(\mathrm{SH}\left(\mathrm{CH}_{2}\right)_{2} \mathrm{NH}_{2}\right.$, thereafter referred to as $\left.\mathrm{C}_{2}-\mathrm{NH}_{2}\right)$, 3-Mercapto-1-propanol $\left(\mathrm{SH}\left(\mathrm{CH}_{2}\right)_{3} \mathrm{OH}, \mathrm{C}_{3^{-}}\right.$ $\mathrm{OH})$, 11-Mercapto-1-undecanol $\left(\mathrm{SH}\left(\mathrm{CH}_{2}\right)_{11} \mathrm{OH}, \mathrm{C}_{11}-\mathrm{OH}\right)$, 3-Mercaptopropionic acid (SH$\left.\left(\mathrm{CH}_{2}\right)_{2} \mathrm{COOH}, \mathrm{C}_{2}-\mathrm{COOH}\right), 11-\mathrm{Mercaptoundecanoic}$ acid $\left(\mathrm{SH}-\left(\mathrm{CH}_{2}\right)_{10} \mathrm{COOH}, \mathrm{C}_{10}-\mathrm{COOH}\right), 1,2-$ Ethanedithiol ( $\left.\mathrm{SH}\left(\mathrm{CH}_{2}\right)_{2} \mathrm{SH}, \mathrm{C}_{2}-\mathrm{SH}\right), 1,9-\mathrm{Nonanedithiol}\left(\mathrm{SH}\left(\mathrm{CH}_{2}\right)_{9} \mathrm{SH}, \mathrm{C}_{9}-\mathrm{SH}\right)$, and 1Dodecanethiol $\left(\mathrm{SH}\left(\mathrm{CH}_{2}\right)_{11} \mathrm{CH}_{3}, \mathrm{C}_{11}-\mathrm{CH}_{3}\right)$. The schematic of the experimental design and the rationale behind it are represented in Fig. 7. This system provides a straightforward way to gauge the effect of surface chemistry on adhesion by keeping the elasticity and largely the capillarity components constant.

We found that, following evaporation-induced assembly (Fig. 7A-C), the stability of the assembled clusters is highly sensitive to the surface chemistry of the fibers. For short-chain molecules, the assembly stability, read out as the percentage of stable clusters, roughly parallels the strengths of the chemical bonds expected to form by the respective functional groups: $\mathrm{H} \cdots \mathrm{NH}_{2} \approx \mathrm{H} \cdots \mathrm{OH}<\mathrm{H} \cdots \mathrm{O}=\mathrm{COH}<\mathrm{RS}-\mathrm{SR}$ (or RS-Au). For each functional group, the cluster stability can be further modulated by varying the carbon chain length. In general, SAMs with longer carbon chains produce more ordered monolayers and hence more stable clusters than their shorter homologs. SAMs terminated with SH groups are also influenced by chain length but show the opposite trend: a long carbon chain (as in $\mathrm{C}_{9}-\mathrm{SH}$ ) nearly eliminates stable clusters. This likely results from the $\mathrm{SH}$ group on the end of the highly flexible carbon chain looping back to bond to the same fiber, exposing mostly weakly bonding hydrocarbon chains rather than $\mathrm{SH}$ groups, and thus showing behavior similar to that of $\mathrm{C}_{11}-\mathrm{CH}_{3}$.

Overall, this sensitivity to surface chemistry provides a straightforward way to fine-tune the assembling system for complete reversibility, high stability, or a wide range of intermediate stabilities simply by choosing an appropriate surface modifier. In these examples, cluster stability directly corresponds to the tendency to undergo spontaneous dissociation when the system dries, as determined by the strength of the adhesion force, $\mathrm{A}_{1}$, relative to the elastic restoring force E (Fig. 7D-F). For example, although no stable clusters are observed for fibers modified with weakly bonding $\mathrm{C}_{11}-\mathrm{CH}_{3} \mathrm{SAMs}$, our real-time monitoring of the evaporation process clearly shows the formation of the four-fiber clusters covering the whole surface during the initial stages of evaporation and their quick disassembly as soon as the sample dries [84].

To program the system for inducible rather than spontaneous reversibility, we take advantage of the fact that the chemical environment can influence the chemical bonds 

directly disrupt the hydrogen bonds between SAM functional groups, while non-polar solvents such as chloroform can act through disturbing first the highly ordered structure of the monolayer, effectively "unzipping" it and, as a result, quickly destroying the tightly knit hydrogen bond network that holds the fibers together. Such a mechanism should apply specifically to relatively weak non-covalent bonds, such as the hydrogen bonds between $\mathrm{COOH}$ or $\mathrm{OH}$ groups. In the terminology presented in Fig. 7, changing the chemical environment can change the adhesion from $A_{1}$ to $A_{2}$, and will induce disassembly if $A_{2}<E$. We found that the stable clusters formed by fibers functionalized with $\mathrm{C}_{11}-\mathrm{OH}, \mathrm{C}_{2}-\mathrm{COOH}$, and $\mathrm{C}_{10}-\mathrm{COOH}$ were easily disassembled by water or organic solvents, such as ethanol, acetone, and chloroform. The rate of disassembly of the clusters decreases in the order that roughly follows the increase of the dielectric constant of the solvent: chloroform (fastest rate) >> acetone > ethanol $\approx$ water, suggesting that "unzipping" the monolayers by non-polar solvents (that likely starts at the regions with a higher monolayer disorder) occurs at a faster rate than the disruption of the fairly tightly connected hydrogen bonding networks by polar and protic solvents. In contrast, the clusters held together by S-S or $\mathrm{S}$-Au bonds remain stable, even after prolonged exposure to these solvents. Significantly, after the solvent-induced disassembly, the clusters can be easily reformed by re-immersing the samples in ethanol and drying.

This fine control over cluster stability allows us to achieve an exceptionally high level of spatial and temporal control over the self-assembly process. Using microcontact printing to pattern the surface with defined regions of strongly bonding SAMs, we have demonstrated that the clustering pattern can be switched on and off selectively. Fig. 8A-F presents still frames from a movie showing one cycle of exposure of a dry patterned micropillar array (Fig. 8a) to ethanol and then to chloroform. The patterned clusters are formed in ethanol (Fig. 8B-C) and then quickly erased by chloroform (Fig. 8D-F). The process can be repeated multiple times with the clusters that are coded on the surface being regenerated and erased, analogous to the reversible assembly and reassembly of the tarsi of the beetle Hemisphaerota cyanea [6] .

Our experimental results demonstrate the critical role that chemical adhesion forces play in determining the stability of self-assembled clusters of microfibers. Clearly, simple modifications of the surface chemistry allow the assemblies to be tuned for nearly any degree of reversibility and selective assembly/disassembly. Changes in the solvent environment can dramatically alter the molecular interactions and lead to a very quick switching between the stable clusters and fully disassembled fibers. We believe that such fine control over stability and reversibility of self-assembly on the mesoscale can lead to a new generation of dynamic materials with tunable adhesive, optical, and other properties[85].

\section{Summary and Outlook}

The advances discussed in this review reveal the tremendous diversity and complexity that can be generated from a simple nanofiber array, just by working with the most basic parameters of the system and manipulating the delicate balance among competing forces. Without any complicated pre-patterning, we program not only whether fibers come together but how they bundle along their length, twist around each other into chiral swirls, assemble into patterned arrays of complex hierarchical architectures, and reversibly disassemble in response to environmental cues. This control derives from a small but powerful set of low-cost benchtop techniques we have developed that allow us to systematically vary multiple features of the fibers 
and array geometry starting from a single master surface. The phenomena we describe are undoubtedly just a small sample of the self-assembly possibilities that can be achieved through these means. With the endless programming permutations and combinations the techniques make available, we can begin to take much greater advantage of the conformational and dynamic versatility that likely make nanoscale fibers such a ubiquitous feature of the natural world.

Modulating the complex interplay between fiber elasticity and surface chemistry will be one of the most critical components of realizing this potential. The unique relationship between surface and mechanical properties that occurs at the nanoscale is, after all, the defining feature that gives nanoscale fibers their versatility. While chemical interactions may dominate at smaller scales and bulk mechanics at larger scales, the subtle balance between them in our system is clearly a major determinant of nearly every aspect of assembly, from shape to chirality to hierarchy to dynamics. Surface chemistry and macroscale mechanics have not traditionally been studied together, but the fabrication and characterization techniques we present provide a straightforward way to fine-tune both the elastic and the surface properties of the fibers independently, through both rational design and empirical calibration of various parameters.

An equally critical design element for developing functional surfaces will be understanding and manipulating the dynamics of the assembly process. We have shown that assembly proceeds through a series of discrete hierarchical stages, with each stage producing a new array of structures with new geometry, symmetry, and mechanical and surface profiles. Each step therefore leads to a distinct surface for either a downstream application or the starting point for another round of assembly. From a general programming perspective, this means that global energy minimization based solely on the initial properties of the surface will often be insufficient for predicting the final structure. The complex kinetics of the continuously evolving shape, mechanical, and other parameters plays a fundamental role and will need to be taken into account. At the same time, the emergence of new surface properties at every step provides an opportunity to use self-assembly principles to design smart, dynamic surfaces. We have shown that hierarchical assembly can run in reverse, and that modifying the fiber surface chemistry can be used to specify the degree of reversibility as well as to introduce responsiveness to changes in the environment. These controls open up the possibility of switching or gradually changing the emergent physical, biological, optical, adhesive, and other properties of the fibrous surface in response to specific stimuli, and of designing surfaces that capture and release particles or generate microfluidic mixing as the fibers associate and dissociate.

The combined self-assembly and general surface fabrication capabilities offered by the approaches presented here provide the foundation for transforming the design of functional fibrous surfaces across a wide range of fields. The fabrication strategies on their own have already shown promise in programming surfaces for controlling bacterial patterning, cell behavior, water and ice repellency, and fluid flow sensing and can be directly applied to dry adhesion, memory and storage, and microfluidic transport processes [45, 56-58]. The capacity to co-program each surface for hierarchical, reversible self-assembly brings each application to a new level of sophisticated design and dynamic, adaptive behavior.

\section{ACKNOWLEDGMENTS}

We would like to thank Dr. Michael Bucaro for helpful discussion on AFM data analysis and Dr. Jiangdong Deng for AFM user advice. This work was partially supported by the Materials 
Research Science and Engineering Center under NSF Award No. DMR-0820484 (fabrication) and by the by DOE under Award No. DE-SC0005247 This work was performed in part at the Center for Nanoscale Systems (CNS) at Harvard University, a member of the National

Nanotechnology Infrastructure Network (NNIN), which is supported by the National Science Foundation under NSF award No. ECS-0335765, and at the MIT's Microsystems Technology Laboratories. 


\section{Figure 1}

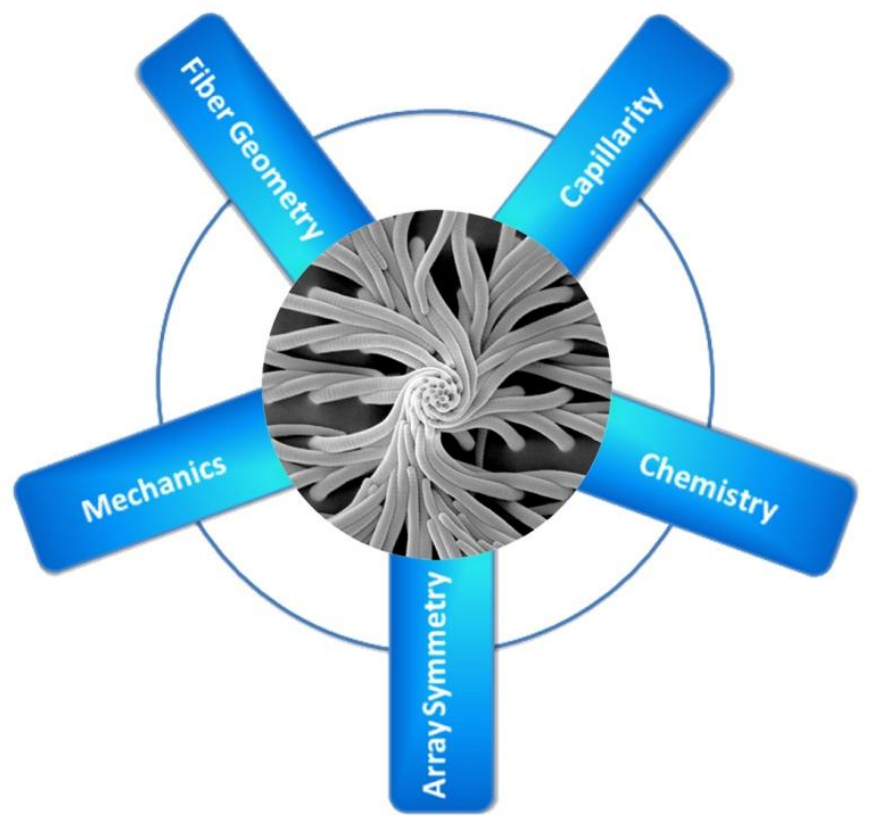

28

29

30

31

32

33

34

35

36

37

38

39

40

41

42

43

44

45

46

47

48

49

50

51

52

53

54

55

56

57

58

59

60

61

62 
Figure 2

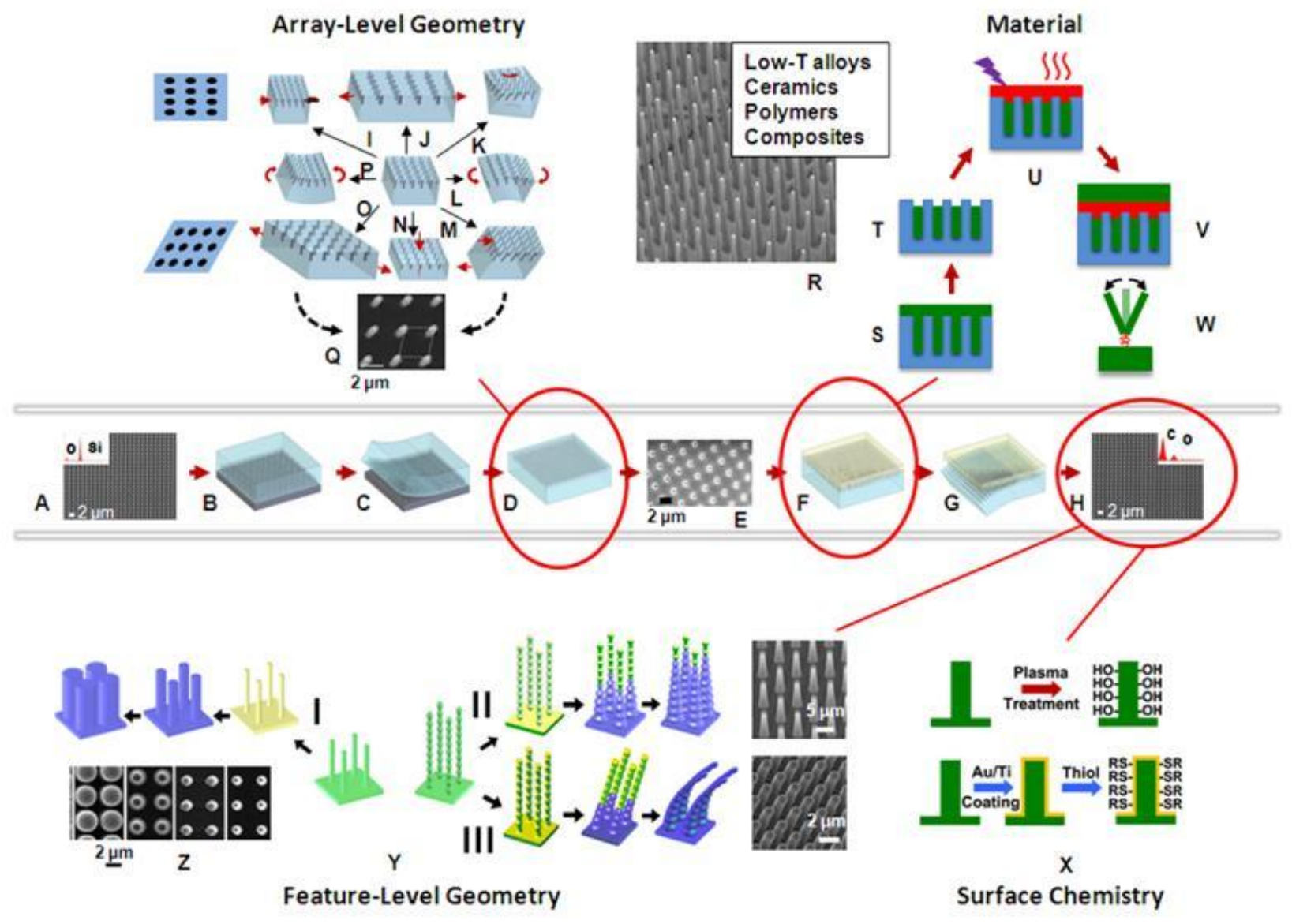


Figure 3
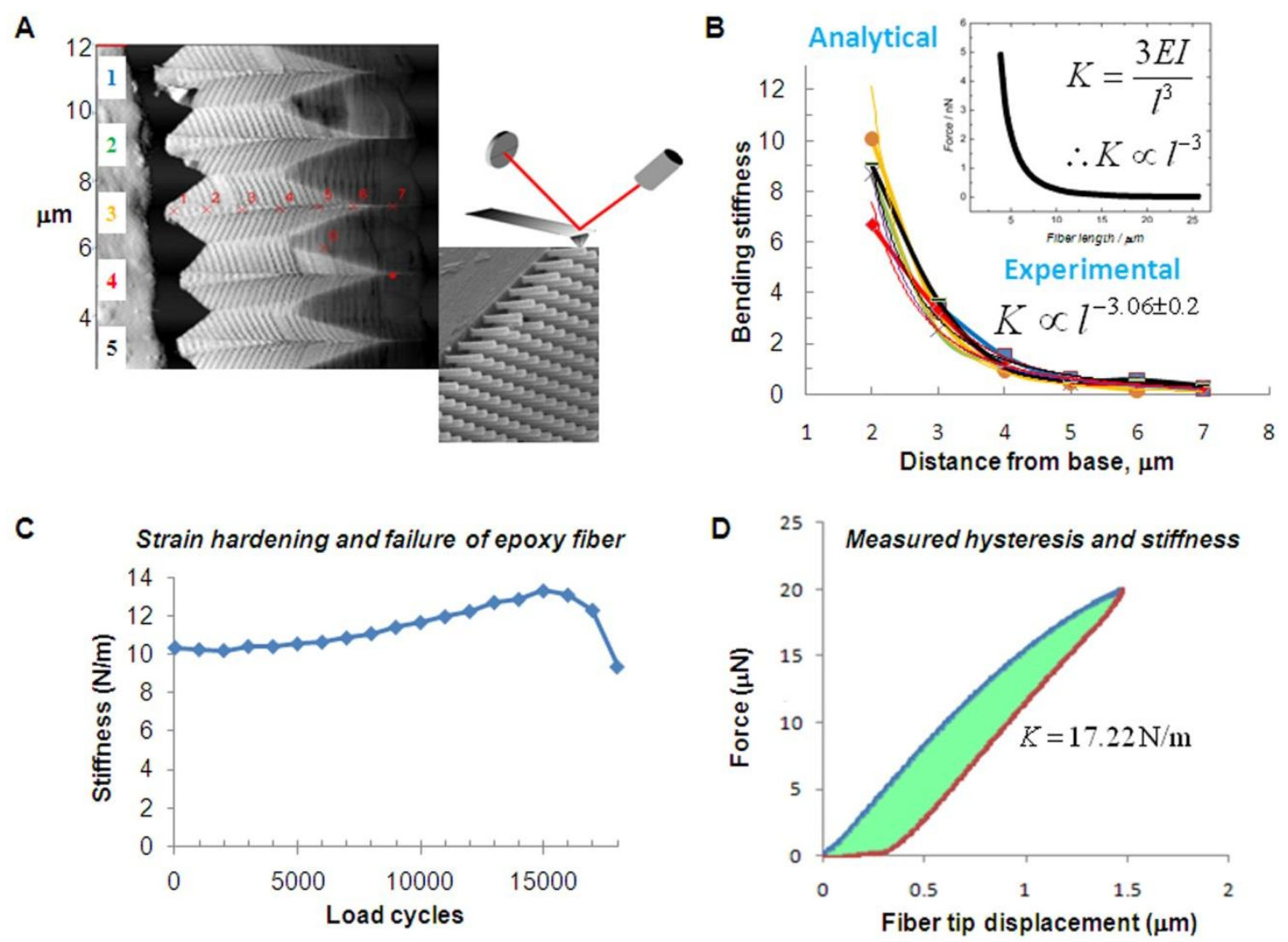
Figure 4.
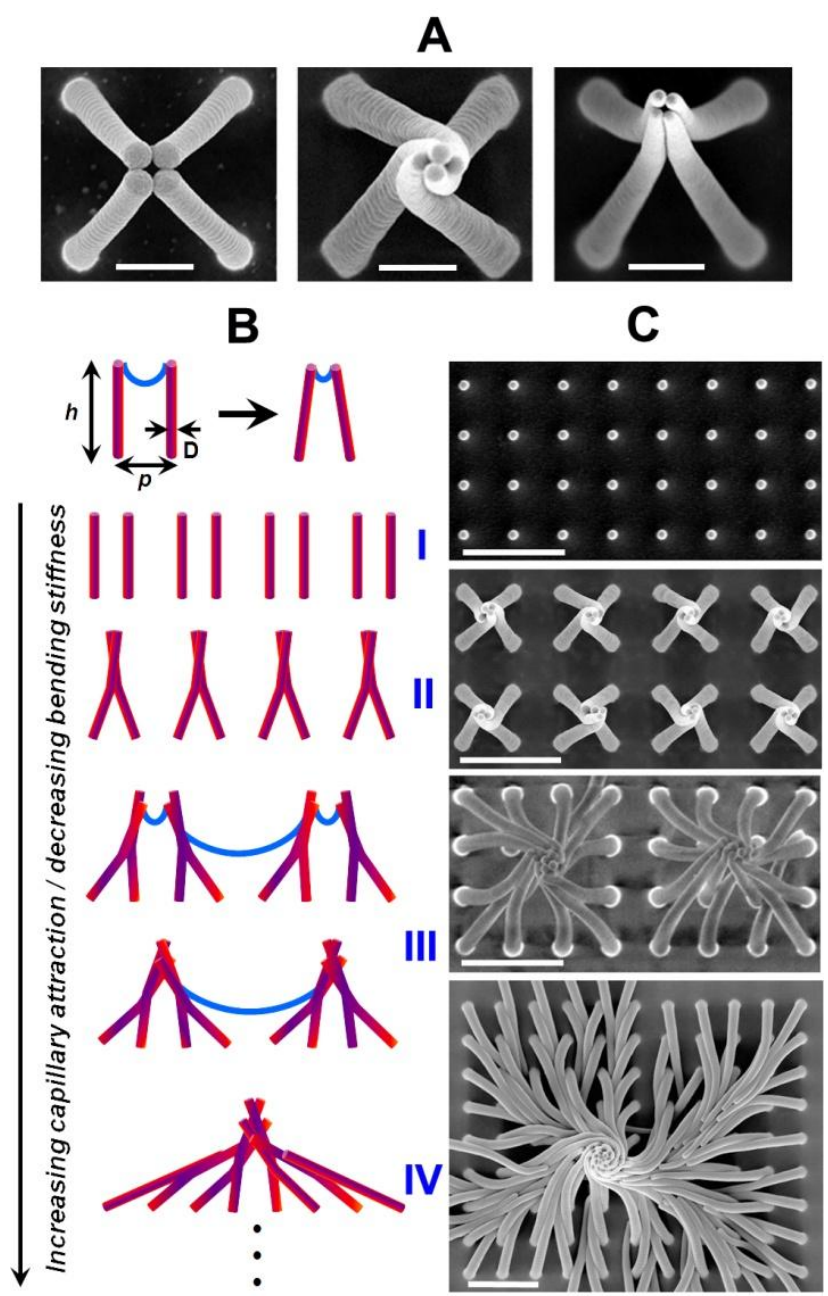

39

40 


\section{Figure 5}

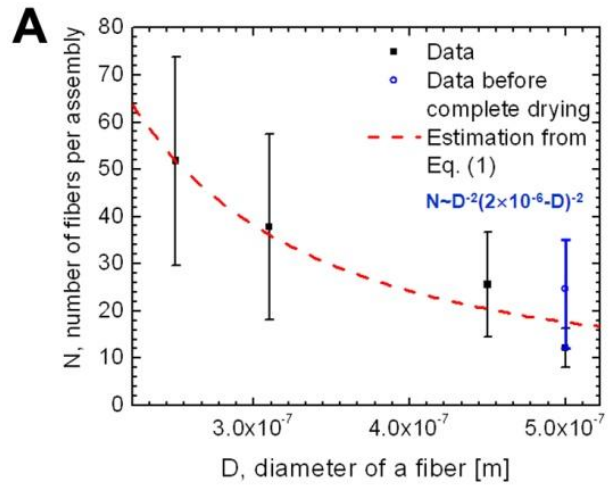

B

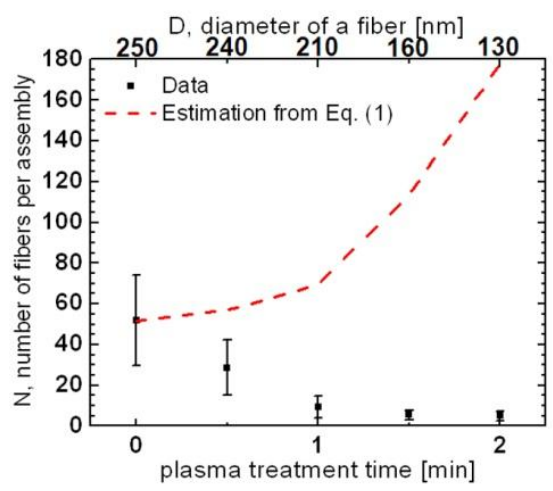

C
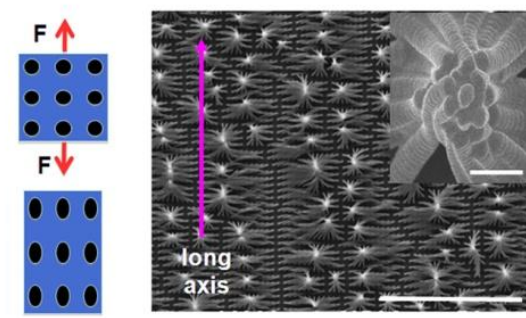

D

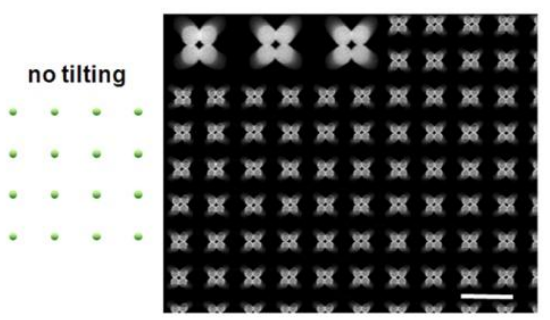

E

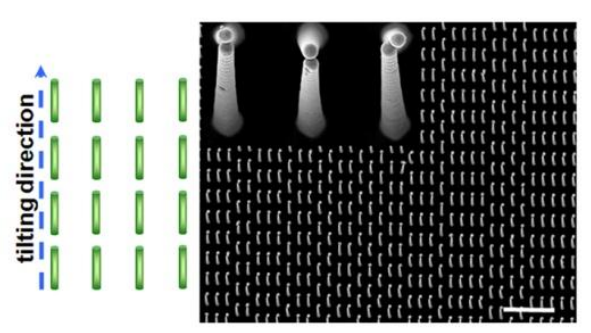

$F$

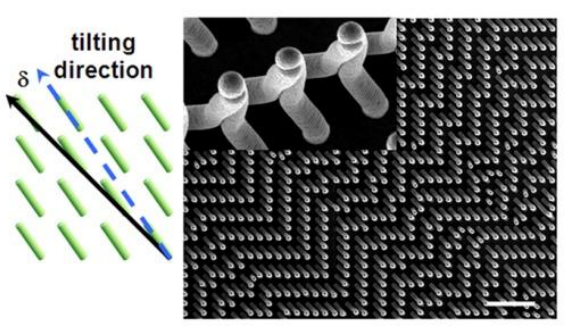


Figure 6.

A

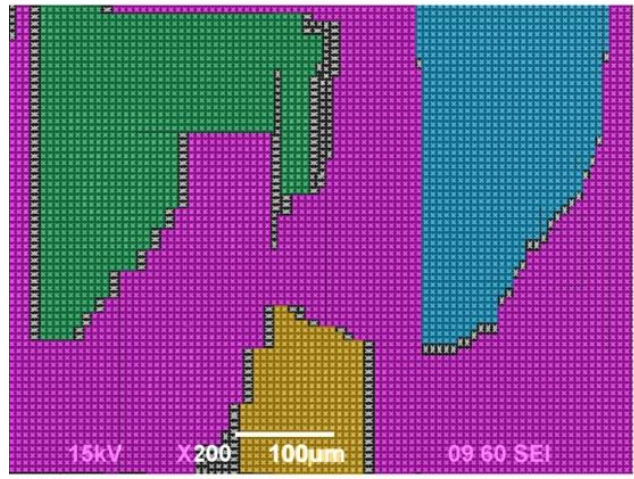

C

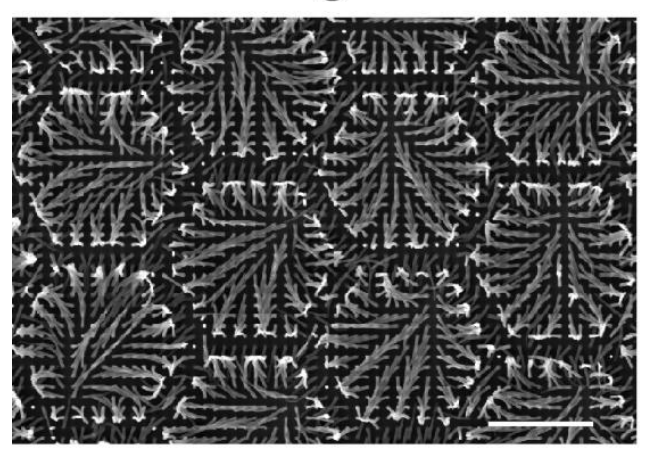

B

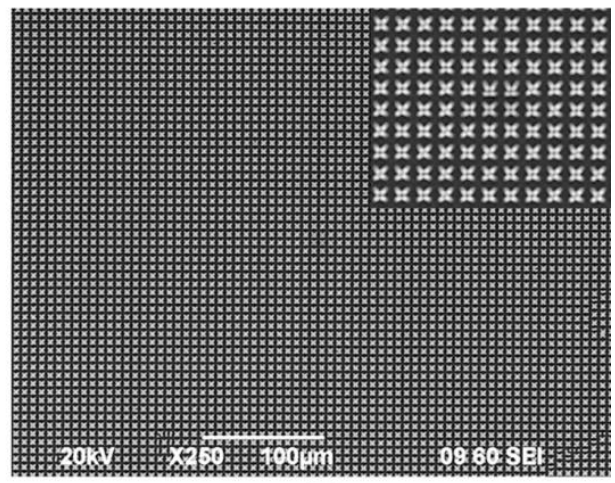

D

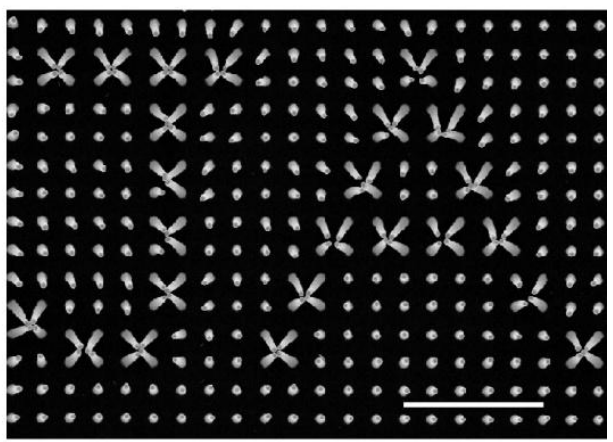




\section{Figure 7.}

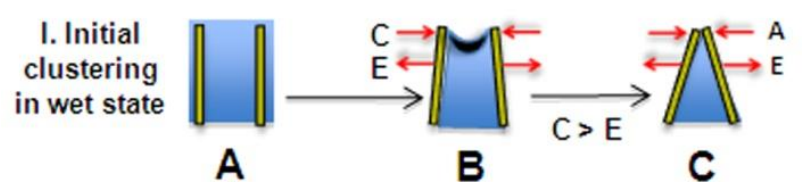

III. Inducing reversible assembly by changing chemical environment
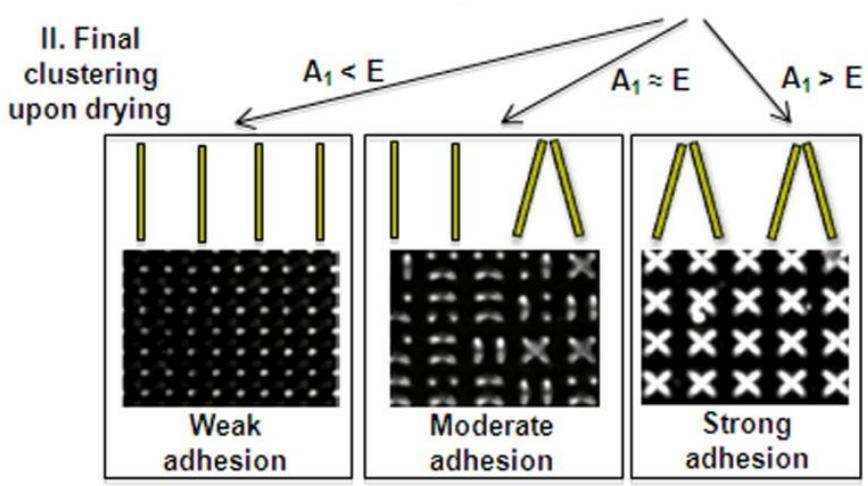

D
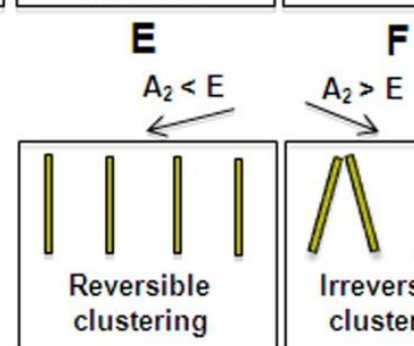

$A_{2}>E$
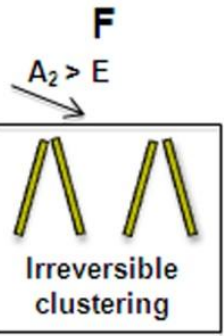

G

H 
Figure 8.

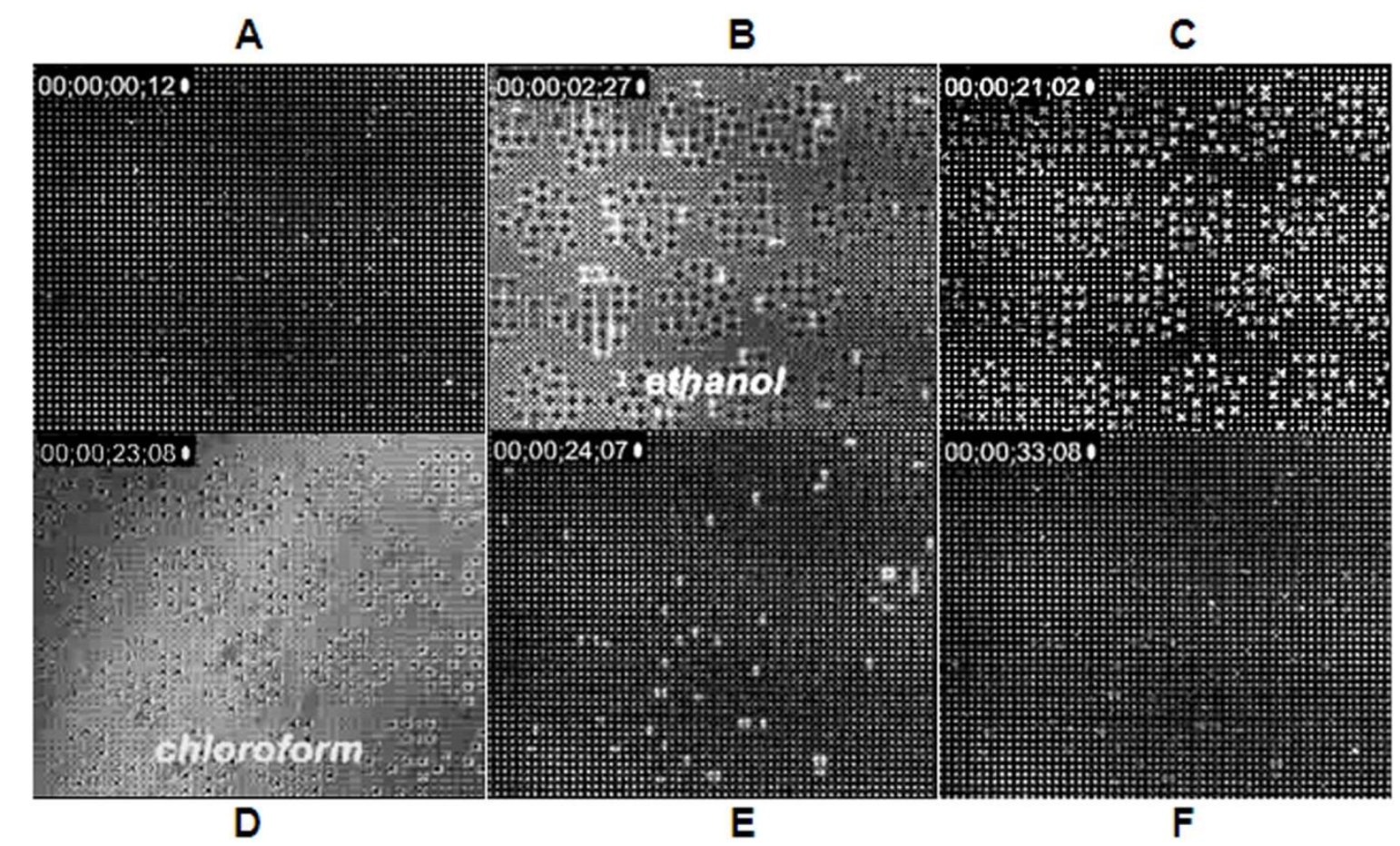

\section{FIGURE CAPTIONS}

Figure 1. Integrative approach to programming nanoscale fiber self-assembly.

Figure 2. The overall fabrication platform for bioinspired arrays of high-aspect-ratio nanostructures. (A) SEM image of an exemplary original nanostructured surface-a silicon master bearing a square array of $8-\mu \mathrm{m}$-high fibers with a diameter of $250 \mathrm{~nm}$ and a pitch of 2 $\mu \mathrm{m}$. The insert is an EDS spectrum. (B) Liquid PDMS or other elastomeric negative mold precursor is poured onto the master, which may be treated with an anti-sticking agent, and cured. (C) The cured PDMS is peeled off from the master. (D) The negative PDMS mold, which contains an array of high-aspect-ratio wells corresponding to the nanofibers of the positive master, can be surface-treated with an anti-sticking agent. (E) SEM image of the PDMS mold, revealing the high-aspect-ratio wells. (F) Liquid precursor (polymer, liquid metal) is poured onto the negative PDMS mold and cured. Alternatively, ceramic precursor may be deposited by atomic layer deposition. (G) The PDMS mold is peeled from the cured positive replica. (H) SEM image of an exemplary nanostructured replica fabricated from epoxy resin. The insert is an EDS spectrum. The replicated structure is geometrically indistinguishable from the master shown in A). (I)-(P) Schematic 3D renderings of various deformations of the PDMS mold, which allow arbitrary array-level geometric control with finely tuned geometries and nontrivial configurations [43]. The unmodified mold (center) can be: (I) compressed along the [100] direction, (J) stretched along the [100] direction, (K) torsioned about the [001] axis, (L) uniformly concavely curved, (M) sheared along the [100] direction, (N) compressed along the [001] direction, (O) 

nanofiber array fabricated using a compound shear and $45^{\circ}$ extension of the mold. (R) Geometric control of the replica via negative mold deformations can be compounded, for example a [110] extension and a shearing-induced tilt. (S) A wide range of materials may be used for the final nanoarray. SEM image is of ceramic nanfibers cast from a PDMS mold by ALD. (T)-(W) Casting of the replica material in liquid form enables arbitrary Young's modulus — at least 5 orders of magnitude - and other material properties. Furthermore, it is possible to fabricate multi-material replicas that provide spatial control of stiffness, magnetization, etc. and introduce functional gradients in the system. [53] As an example, rigid fibers may be flexibly tethered to a rigid substrate via an elastomeric layer for controlled actuation only at the base. (X) Surface chemistry can be modified independently of the materials choice by plasma treatment or thiolation. (Y) Schematics showing the step-by-step feature-level geometric control of structural transformation by electrodeposition on patterned substrates (STEPS). The dimensions of the features can be finely tailored independent of the array parameters using this part of the fabrication platform [45]. The three schemes employ either a sputter-coated metal electrode (type I), evaporated metal electrodes from the top (type II), or evaporated metal electrodes at an angle (type III). Green: parent substrate, yellow: metal coating, blue: polypyrrole. SEM insets show the gradually increasing diameter of cylindrical fibers and the decreasing space between adjacent fibers as type I deposition progresses from right to left; an array of conical nanostructures transformed by type II from cylindrical fibers; directionally bent conical structures transformed from upright cylindrical fibers using STEPS type III.

Figure 3. Empirical characterization of fiber mechanical properties. (A) Sample preparation for single fiber measurement by AFM and scan of five silicon nanofibers $(\mathrm{h}=6.8$ $\left.\mu \mathrm{m}, \mathrm{r}_{\text {base }}=150 \mathrm{~nm}, \mathrm{r}_{\text {tip }}=90 \mathrm{~nm}\right)$. A cross-section is mounted at 90 degrees such that the AFM cantilever tip can test the mechanical properties of single nanofeatures in bending. The contactmode scan shows the $1 \mu \mathrm{m}$ interval locations along five silicon nanofibers where force and displacement were measured to validate mechanical characterization by AFM in reference to Euler beam theory. (B) Empirical best-fit effective stiffness curves for $1 \mu \mathrm{m}$ interval locations along the lengths of the five silicon nanofibers. These curves show an average decay of power $\sim 3$, in excellent registry with the inverse cubic relation of effective stiffness to cantilever length in the analytically predicted curve (inset). This helps confirm that the AFM-based single nanofeature characterization approach is viable and accurate. (C) The fatigue life of a single epoxy fiber $\left(1=9.0 \mu \mathrm{m}, \mathrm{r}_{\text {base }}=1.05 \mu \mathrm{m}, \mathrm{r}_{\text {tip }}=0.85 \mu \mathrm{m}\right)$, important for long-term applications, is characterized by AFM. The nanofiber monotonically increases in stiffness by $30.6 \%$ over 15,000 load cycles applied at $16 \mathrm{~Hz}$ and $91 \mu \mathrm{m} / \mathrm{s}$, consistent with strain hardening behavior, which decreases its sensitivity. After this point the stiffness rapidly drops off and structural failure occurs. (D) Using AFM characterization, the effective stiffness of HAR epoxy microfibers is measured to be $17.22 \mathrm{~N} / \mathrm{m}$, and the hysteresis of the load-unload curve is measured, highlighted in green. Such minor hysteresis of $32 \%$ reveals that epoxy nanofibers are primarily elastic in their behavior in this bending range.

Figure 4. Assembly of fibers into diverse shapes and hierarchical architectures. (A) Clusters of fibers connected at the tips (left), twisted into a chiral bundle (center), or zipped together (right). Scale bar $=1 \mu \mathrm{m}$. (B) Schematic and (C) corresponding SEM images showing the hierarchical assembly of fiber arrays into higher order chiral structures. Blue lines in the 

stepwise kinetics of hierarchical assembly.

Figure 5. Effects of changing the properties of fibers on size and shape of the assembly. (A) Plot showing the relation between the fiber diameter and the number of fibers per assembled cluster. (B) Plot of the number of fibers per assembled cluster as a function of plasma treatment time. Since the plasma treatment simultaneously increases fiber surface adhesion and decreases diameter, the corresponding fiber diameters are shown on the top axis of the graph for each plasma treatment. Note that the number of fibers per assembly decreases with increasing plasma treatment time even though the diameter of fibers decreases. (C)-(F) Left column shows schematics of the method used to fabricate various anisotropic fiber arrays and right column shows SEM images of the corresponding assemblies formed from each array. The insets show high-magnification images of the corresponding SEM images. (C) Fibers with elliptical cross section and a rectangular lattice form anisotropic cluster shapes. The long axis indicates the direction in which the tensile force was applied to an elastomeric mold. (Scale bar $=50 \mu \mathrm{m}$ ) The inset shows the elliptical cross section of the fiber. (Scale bar $=1 \mu \mathrm{m}$ ) (D) Upright fibers with a square lattice array form four-fiber clusters. (E) Fibers tilted along a lattice direction form an array of achiral two-fiber clusters. (F) Fibers tilted slightly off a diagonal lattice direction form chiral two-fiber clusters with a uniform handedness. Scale bars $=10 \mu \mathrm{m}$ for (C)-(E).

Figure 6. Long-range order of assembly. (A) Large area view of the assembled fibers showing multiple domains and domain boundaries represented in color. (Scale bar $=100 \mu \mathrm{m})(\mathrm{B})$ SEM image showing a single domain with uniform long-range order formed by controlling movement of the meniscus during evaporation. (Scale bar $=100 \mu \mathrm{m}$ ) The inset shows the magnified view of the corresponding image showing uniform domain formation. (C) SEM image of fibers assembled under a honeycomb mask. (Scale bars $=20 \mu \mathrm{m}$ ) (D) Writing of arbitrary letters in nanofiber arrays by repeated exposure to an electron beam in spot mode. (Scale bar $=$ $10 \mu \mathrm{m})$

Figure 7. Scheme of the experimental set-up for the study of adhesion effects on the self-assembly of microfibers. When fully immersed in a wetting liquid, microfibers are initially in an upright position and do not interact (A). During solvent evaporation (B), the fibers are brought together $(\mathrm{C})$ if the capillary force $\mathrm{C}$ that drives bending is stronger than the restoring elastic force $\mathrm{E}(\mathrm{C}>\mathrm{E})$. After the solvent dries, $\mathrm{C}$ is lost and the only force opposing $\mathrm{E}$ is the adhesion force $\mathrm{A}$. Intermolecular bonds between assembled fibers are expected to have formed at this stage and to contribute to A. Clusters will disassemble, if $\mathrm{A}_{1}<\mathrm{E}$ (where $\mathrm{A}_{1}$ denotes intefiber adhesion resulting from exposure to Solvent 1) (D); be only moderately stable if $A_{1} \approx E(E)$; or be very stable if $A_{1}>E(F)$. Introduction of different solvents can change the chemical environment and affect adhesion $\left(\mathrm{A}_{1} \rightarrow \mathrm{A}_{2}\right)$ by altering the bonding network between fibers, allowing one to induce reversibility of self-assembly $(\mathrm{G})$. Solvent 2 will induce disassembly if $A_{2}<E(G)$, but clusters will remain stable if $A_{2}>E(H)$. For the array of fibers, the magnitude of $A_{1}$ or $A_{2}$ relative to $E$ is directly read out as the percentage of stable clusters, allowing quantitative comparison of the adhesion force for different surface modifications and chemical environments. Representative micrographs (D-F, top view; vide infra) illustrate the resulting assembly patterns. 
Figure 8. Still frames from a movie showing reversible localized cluster formation and erasure in a microfiber array patterned with regions functionalized with $\mathbf{C}_{10}-\mathbf{C O O H}$. (A) Dry microfiber array with no clusters. (B) Capillary-induced patterned clustering in the presence of ethanol. (C) Stable patterned clusters after evaporation of ethanol. (D) The clustered microfiber array immediately after exposure to chloroform. (E) Almost instantaneous complete disassembly of the clusters in chloroform. (F) Dry, unclustered array after evaporation of chloroform. The reversible clustering can be triggered multiple times by alternating exposure of the array to ethanol and chloroform. Frame dimensions are $470 \mu \mathrm{m} \times 385 \mu \mathrm{m}$.

Table 1. Deformation-induced changes in the geometry of the replicated nanostructures [a]

\begin{tabular}{|c|c|c|c|c|c|}
\hline Parameter & Deformatic & Type & & & \\
\hline & $\begin{array}{l}\text { No } \\
\text { deformation }\end{array}$ & $\begin{array}{l}\text { Stretching / } \\
\text { compressing } \\
\text { along [100] }\end{array}$ & $\begin{array}{l}\text { Stretching / } \\
\text { compressing [110] }\end{array}$ & $\begin{array}{l}\text { Shearing } \\
\text { along [100] }\end{array}$ & $\begin{array}{l}\text { Compression } \\
\text { along [001] }\end{array}$ \\
\hline $\mathbf{a}$ & $a_{0}$ & $1 / 3 a_{0}<a<3 a_{0}$ & $a_{0}<a<2.1 a_{0}$ & $a=a_{0}$ & $a \cong a_{0}$ \\
\hline b & $b_{0}=a_{0}$ & $3 a_{0}>b>1 / 3 a_{0}$ & $b=a$ & $b=a_{0}$ & $b=a$ \\
\hline $\boldsymbol{\theta}$ & $\theta_{0}=90^{\circ}$ & $\theta=\theta_{0}$ & $12.5^{\circ}<\theta<167.5^{\circ}$ & $\theta=\theta_{0}$ & $\theta=\theta_{0}$ \\
\hline $\begin{array}{c}\text { Tilt }(t) \\
1 \\
1 \\
t\end{array}$ & $t_{0}=0$ & $t=t_{0}$ & $t=t_{0}$ & $0<t<63.4^{\circ}$ & $t=t_{0}$ \\
\hline $\begin{array}{l}\text { Post } \\
\text { lengths }(l)\end{array}$ & $l_{0}$ & $l \cong l_{0}$ & $l \cong l_{0}$ & $l_{0}<l \leq \sqrt{5} l_{0}$ & $1 / 3 l_{0}<l<l_{0}$ \\
\hline $\begin{array}{l}\text { Cross } \\
\text { section } \\
r_{1} \mid\end{array}$ & $r_{1}=r_{2}=r_{0}$ & $r_{1}<r_{2}$ & $r_{1}<r_{2}$ & $r_{1}=r_{2}=r_{0}$ & $r_{1}=r_{2}>r_{0}$ \\
\hline $\begin{array}{l}\text { 2D array } \\
\text { symmetry }\end{array}$ & $\begin{array}{l}\text { square } \\
\bigcirc \quad \bigcirc \\
\bigcirc \quad \bigcirc\end{array}$ & 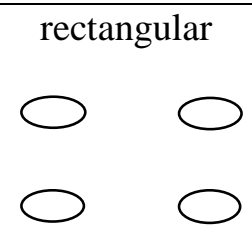 & rhombic & 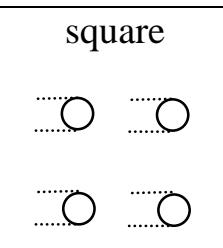 & $\begin{array}{l}\text { square } \\
\bigcirc \bigcirc \\
\bigcirc \bigcirc\end{array}$ \\
\hline
\end{tabular}

[a] The calculations were made using the reported PDMS extendibility parameter of $300 \%$ and Poisson's ratio $v=0.5$ [87]. 


\section{References}

[1] S.H. Li, Q.Y. Zeng, Y.L. Xiao, S.Y. Fu, B.L. Zhou, Mat. Sci. Eng. C-Biomim., 3 (1995) 125130.

[2] H. Lichtenegger, M. Muller, O. Paris, C. Riekel, P. Fratzl, J. Appl. Crystallogr., 32 (1999) $1127-1133$.

[3] W. Wagermaier, H.S. Gupta, A. Gourrier, M. Burghammer, P. Roschger, P. Fratzl, Biointerphases, 1 (2006) 1-5.

[4] A.S. Kozlov, J. Baumgart, T. Risler, C.P.C. Versteegh, A.J. Hudspeth, Nature, 474 (2011) 376-379.

[5] T. Sanchez, D. Welch, D. Nicastro, Z. Dogic, Science, 333 (2011) 456-459.

[6] T. Eisner, D.J. Aneshansley, Proc. Natl. Acad. Sci. USA, 97 (2000) 6568-6573.

[7] C.G. Begley, S. Lipkowitz, V. Gobel, K.A. Mahon, V. Bertness, A.R. Green, N.M. Gough, I.R. Kirsch, Proc. Natl. Acad. Sci. USA, 89 (1992) 38-42.

[8] T.J. Richmond, C.A. Davey, Nature, 423 (2003) 145-150.

[9] B. Pokroy, S.H. Kang, L. Mahadevan, J. Aizenberg, Science, 323 (2009) 237-240.

[10] T.K. Ghosh, L. Eadie, J. R. Soc. Lond. Interface, 8 (2011) 761-775.

[11] W. Barthlott, C. Neinhuis, Planta, 202 (1997) 1-8.

[12] X. Gao, L. Jiang, Nature, 432 (2004) 36.

[13] E. Arzt, S. Gorb, R. Spolenak, Proc. Natl. Acad. Sci. USA, 100 (2003) 10603-10606.

[14] N.A. Oritsland, D.M. Lavigne, Comp. Biochem. Phys. A, 53 (1976) 327-330.

[15] A.S. Shah, Y. Ben-Shahar, T.O. Moninger, J.N. Kline, M.J. Welsh, Science, 325 (2009) 1131-1134.

[16] B. Guirao, J.F. Joanny, Biophys J., 92 (2007) 1900-1917.

[17] J. Branscomb, A. Alexeev, Soft Matter, 6 (2010) 4066-4069.

[18] A. Vilfan, Biophys J., 97 (2009) 1130-1137.

[19] A.R. Shields, B.L. Fiser, B.A. Evans, M.R. Falvo, S. Washburn, R. Superfine, Proc. Natl. Acad. Sci. USA, 107 (2010) 15670-15675.

[20] A.K. Geim, S.V. Dubonos, I.V. Grigorieva, K.S. Novoselov, A.A. Zhukov, S.Y. Shapoval, Nature Mater., 2 (2003) 461-463.

[21] T.N. Krupenkin, J.A. Taylor, T.M. Schneider, S. Yang, Langmuir, 20 (2004) 3824-3827.

[22] A. Sidorenko, T. Krupenkin, J. Aizenberg, J. Mater. Chem., 18 (2008) 3841-3846.

[23] C. Dorrer, J. Ruhe, Langmuir, 24 (2008) 6154-6158.

[24] P. Kim, L.D. Zarzar, M. Khan, M. Aizenberg, J. Aizenberg, in: SPIE Photonics West,

SPIE, San Francisco, CA, 2011, pp. 792705-792711.

[25] F.S. Ou, M. Hu, I. Naumov, A. Kim, W. Wu, A.M. Bratkovsky, X.M. Li, R.S. Williams, Z.Y. Li, Nano Lett., 11 (2011) 2538-2542.

[26] T. Stegmaier, M. Linke, H. Planck, Phil. Trans. R. Soc. A, 367 (2009) 1749-1758.

[27] A. Hilfinger, F. Julicher, Phys. Biol., 5 (2008).

[28] C.Y. Hui, N.J. Glassmaker, T. Tang, A. Jagota, J. R. Soc. Lond. Interface, 1 (2004) 35-48.

[29] C.Y. Hui, A. Jagota, Y.Y. Lin, E.J. Kramer, Langmuir, 18 (2002) 1394-1407.

[30] P. Roca-Cusachs, F. Rico, E. Martinez, J. Toset, R. Farre, D. Navajas, Langmuir, 21 (2005) 5542-5548.

[31] D. Chandra, S. Yang, Acc. Chem. Res., 43 (2010) 1080-1091.

[32] N.J. Glassmaker, A. Jagota, C.Y. Hui, J. Kim, J. R. Soc. Lond. Interface, 1 (2004) 23-33. 
[33] D. Chandra, S. Yang, A.A. Soshinsky, R.J. Gambogi, ACS Appl. Mater. Interfaces, 1 (2009) 1698-1704.

[34] A. Kim, F.S. Ou, D.A.A. Ohlberg, M. Hu, R.S. Williams, Z.Y. Li, J. Am. Chem. Soc., 133 (2011) 8234-8239.

[35] M.F.L. De Volder, S.J. Park, S.H. Tawfick, D.O. Vidaud, A.J. Hart, J. Micromech. Microeng., 21 (2011) 045033.

[36] H. Duan, J.K.W. Yang, K.K. Berggren, Small, DOI: 10.1002/smll.201100892.

[37] D. Wu, Q.D. Chen, B.B. Xu, J. Jiao, Y. Xu, H. Xia, H.B. Sun, Appl. Phys. Lett., 95 (2009). [38] Y.P. Zhao, J.G. Fan, Appl. Phys. Lett., 88 (2006) 103123.

[39] H.G. Duan, K.K. Berggren, Nano Lett., 10 (2010) 3710-3716.

[40] M. De Volder, S.H. Tawfick, S.J. Park, D. Copic, Z. Zhao, W. Lu, A.J. Hart, Adv. Mater., 22 (2010) 4384-4389.

[41] M. Nosonovsky, Langmuir, 23 (2007) 3157-3161.

[42] X.M. Zhao, Y. Xia, G.M. Whitesides, J. Mater. Chem., 7 (1997) 1069-1074.

[43] B. Pokroy, A.K. Epstein, M.C.M. Persson-Gulda, J. Aizenberg, Adv. Mater., 21 (2009) 463469.

[44] A.K. Epstein, Aizenberg, J., Mater. Res. Soc. Symp. Proc., 1236E (2010) 1236-SS12091207

[45] P. Kim, A.K. Epstein, M. Khan, L.D. Zarzar, D.J. Lipomi, G.M. Whitesides, J. Aizenberg, Nano Lett., DOI: 10.1021/nl200426g.

[46] M. Byun, W. Han, B. Li, S.W. Hong, J.W. Cho, Q.Z. Zou, Z.Q. Lin, Small, 7 (2011) 16411646.

[47] A.K. Epstein, B. Pokroy, A. Seminara, J. Aizenberg, Proc. Natl. Acad. Sci. USA, 108

(2011) 995-1000.

[48] S.A. McAuley, H. Ashraf, L. Atabo, A. Chambers, S. Hall, J. Hopkins, G. Nicholls, J. Phys. D: Appl. Phys., 34 (2001) 2769-2774.

[49] R. Ruibal, V. Ernst, J. Morphol., 117 (1965) 271-294.

[50] K. Autumn, A. Dittmore, D. Santos, M. Spenko, M. Cutkosky, J. Exp. Biol., 209 (2006) 3569-3579.

[51] J. Aizenberg, V.C. Sundar, A.D. Yablon, J.C. Weaver, G. Chen, Proc. Natl. Acad. Sci. USA, 101 (2004) 3358-3363.

[52] V.C. Sundar, A.D. Yablon, J.L. Grazul, M. Ilan, J. Aizenberg, Nature, 424 (2003) 899-900.

[53] A.K. Epstein, Aizenberg, J., Mater. Res. Soc. Symp. Proc. , 1236E (2010) 1236-SS12091207

[54] M.J. McHenry, S.M. van Netten, J. Exp. Biol., 210 (2007) 4244-4253.

[55] C. Chang, Y.F. Wang, Y. Kanamori, J.J. Shih, Y. Kawai, C.K. Lee, K.C. Wu, M. Esashi, J. Micromech. Microeng., 15 (2005) 580.

[56] A.I. Hochbaum, J. Aizenberg, Nano Lett., 10 (2010) 3717-3721.

[57] C.J. Bettinger, J.T. Borenstein, R. Langer, Angew. Chem. Int. Ed., 48 (2009) 5406-5415.

[58] L. Mishchenko, B. Hatton, V. Bahadur, J.A. Taylor, T. Krupenkin, J. Aizenberg, ACS

Nano, (2010).

[59] A.R. Ragab, S.E.A. Bayoumi, Engineering Solid Mechanics: Fundamentals and Applications, CRC Press, Boca Raton, FL, 1998.

[60] K. Autumn, C. Majidi, R.E. Groff, A. Dittmore, R. Fearing, J. Exp. Biol., 209 (2006) 35583568 . 
[61] T. Tong, Y. Zhao, L. Delzeit, A. Kashani, M. Meyyappan, A. Majumdar, Nano Lett., 8 (2008) 511-515.

[62] L.F. Wang, C. Ortiz, M.C. Boyce, J. Eng. Mater.-T. ASME, 133 (2011).

[63] B. Schubert, J. Lee, C. Majidi, R.S. Fearing, J. R. Soc. Lond. Interface, 5 (2008) 845.

[64] Y.H. Xiu, Y. Liu, D.W. Hess, C.P. Wong, Nanotechnology, 21 (2010) 155705.

[65] J.H. Song, X.D. Wang, E. Riedo, Z.L. Wang, Nano Lett., 5 (2005) 1954-1958.

[66] B. Wu, A. Heidelberg, J.J. Boland, Nature Mater., 4 (2005) 525-529.

[67] S. Cuenot, C. Fretigny, S. Demoustier-Champagne, B. Nysten, Phys. Rev. B, 69 (2004).

[68] H. Kahn, R. Ballarini, R.L. Mullen, A.H. Heuer, Proc. R. Soc. A, 455 (1999) 3807-3823.

[69] H.G.H. van Melick, H.E.H. Meijer, L.E. Govaert, Polymer, 44 (2003) 2493-2502.

[70] R. Chen, M.C. Lu, V. Srinivasan, Z. Wang, H.H. Cho, A. Majumdar, Nano Lett., 9 (2009) 548-553.

[71] K.K.S. Lau, J. Bico, K.B.K. Teo, M. Chhowalla, G.A.J. Amaratunga, W.I. Milne, G.H. McKinley, K.K. Gleason, Nano Lett., 3 (2003) 1701-1705.

[72] C. Journet, S. Moulinet, C. Ybert, S.T. Purcell, L. Bocquet, Europhys. Lett., 71 (2005) 104109.

[73] N. Bernardino, S. Dietrich, ACS Appl. Mater. Interfaces, 2 (2010) 603-604.

[74] N. Rubin, E. Perugia, M. Goldschmidt, M. Fridkin, L. Addadi, J. Am. Chem. Soc., 130 (2008) 4602-4603.

[75] K. Tsuda, A. Alam, T. Harada, T. Yamaguchi, N. Ishii, T. Aida, Angew. Chem. Int. Ed., 46 (2007) 8198-8202.

[76] M. Ziegler, A.V. Davis, D.W. Johnson, K.N. Raymond, Angew. Chem. Int. Ed., 42 (2003) 665-668.

[77] S.H. Kang, B. Pokroy, L. Mahadevan, J. Aizenberg, ACS Nano, 4 (2010) 6323-6331.

[78] J. Bico, B. Roman, L. Moulin, A. Boudaoud, Nature, 432 (2004) 690-690.

[79] F. Chiodi, B. Roman, J. Bico, Europhys. Lett., 90 (2010) 44006.

[80] J. Paulose, D.R. Nelson, J. Aizenberg, Soft Matter, 6 (2010) 2421-2434.

[81] S.H. Kang, N. Wu, J. Aizenberg, manuscript in preparation.

[82] S.H. Kang, N. Wu, A. Grinthal, J. Aizenberg, Phys. Rev. Lett., 107 (2011) 177802.

[83] A. Seminara, B. Pokroy, S.H. Kang, M.P. Brenner, J. Aizenberg, Phys. Rev. B, 83 (2011) 235438.

[84] M. Matsunaga, M. Aizenberg, J. Aizenberg, J. Am. Chem. Soc., 133 (2011) 5545-5553. [85] After the submission of this review, a paper appeared that described a closely related micropillar assembly through modulation of adhesion by patterned vapor deposition of polymer coatings [86].

[86] B. Chen, S. Seidel, H. Hori, M. Gupta, ACS Appl. Mater. Interfaces, 3 (2011) 4201-4205. [87] J.C. Lotters, W. Olthuis, P.H. Veltink, P. Bergveld, J. Micromech. Microeng., 7 (1997) 145147. 
Figure 1.jpg

Click here to download high resolution image

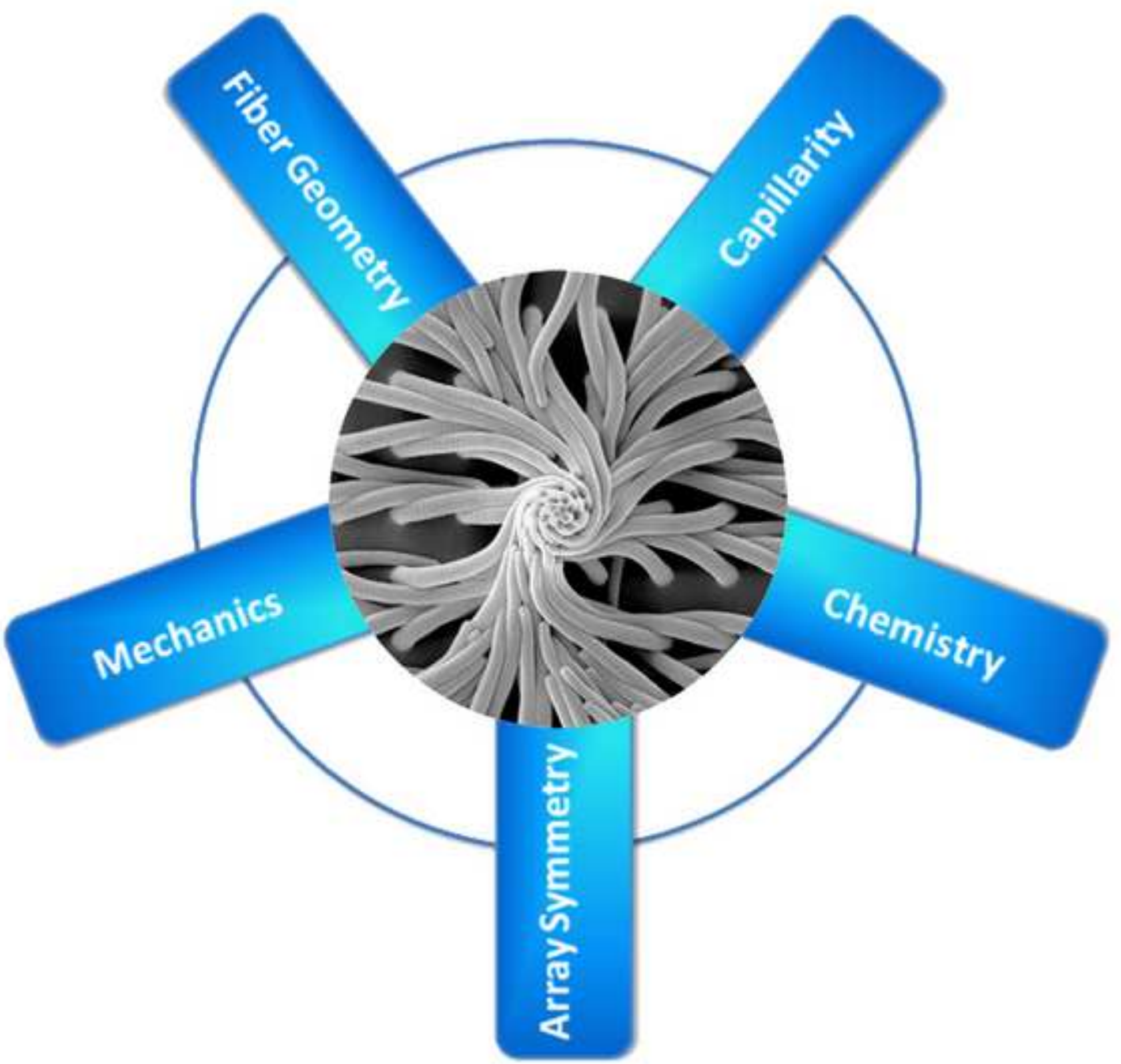




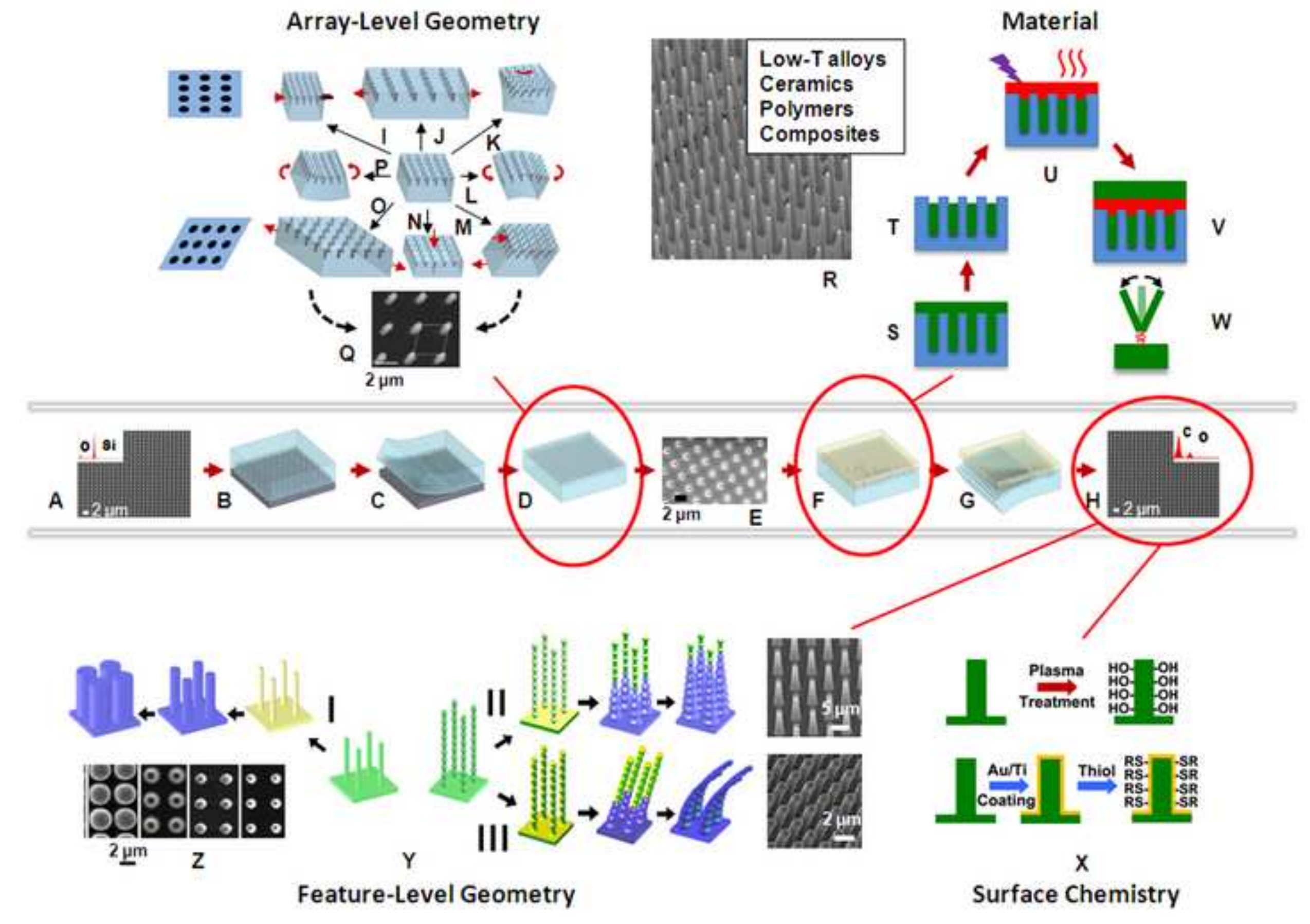


A

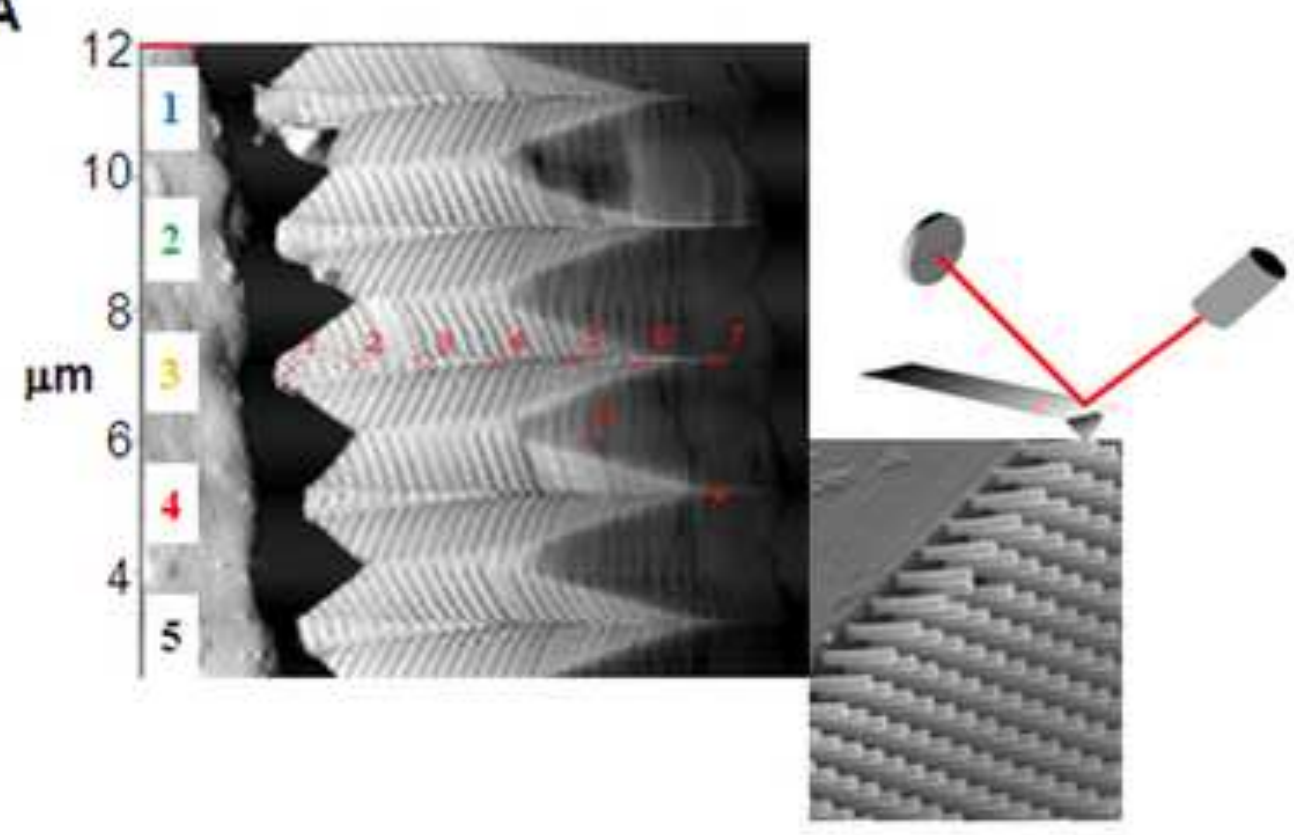

C

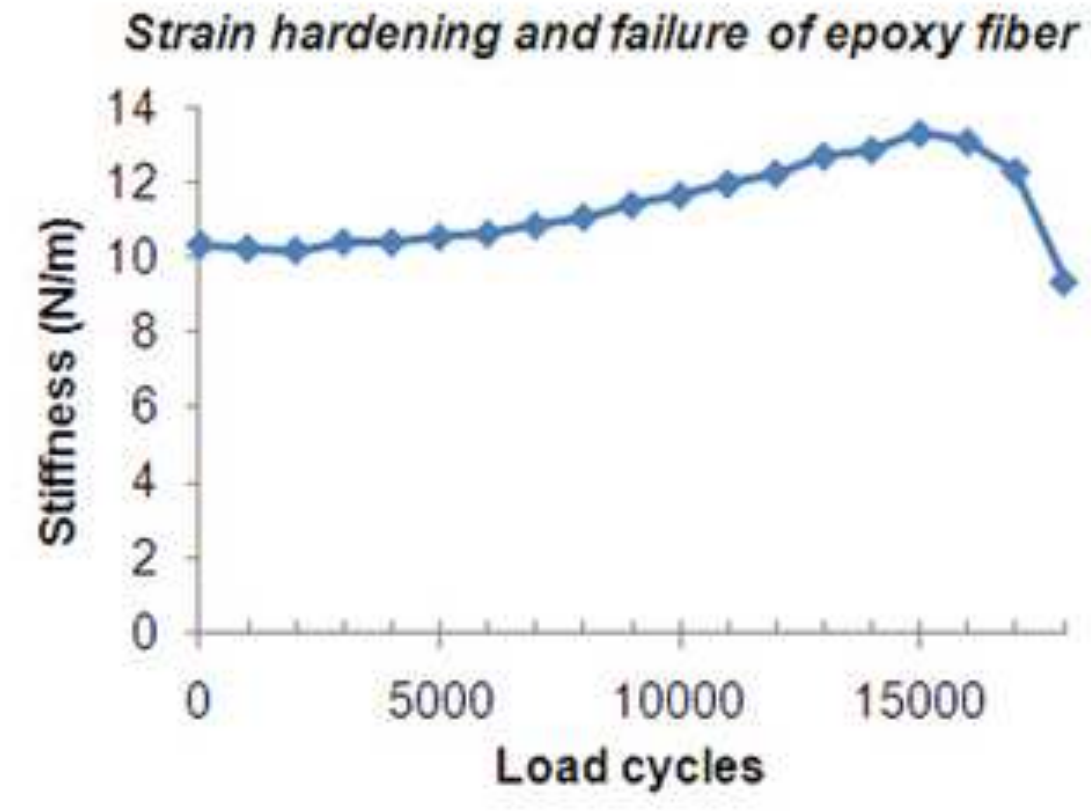

B
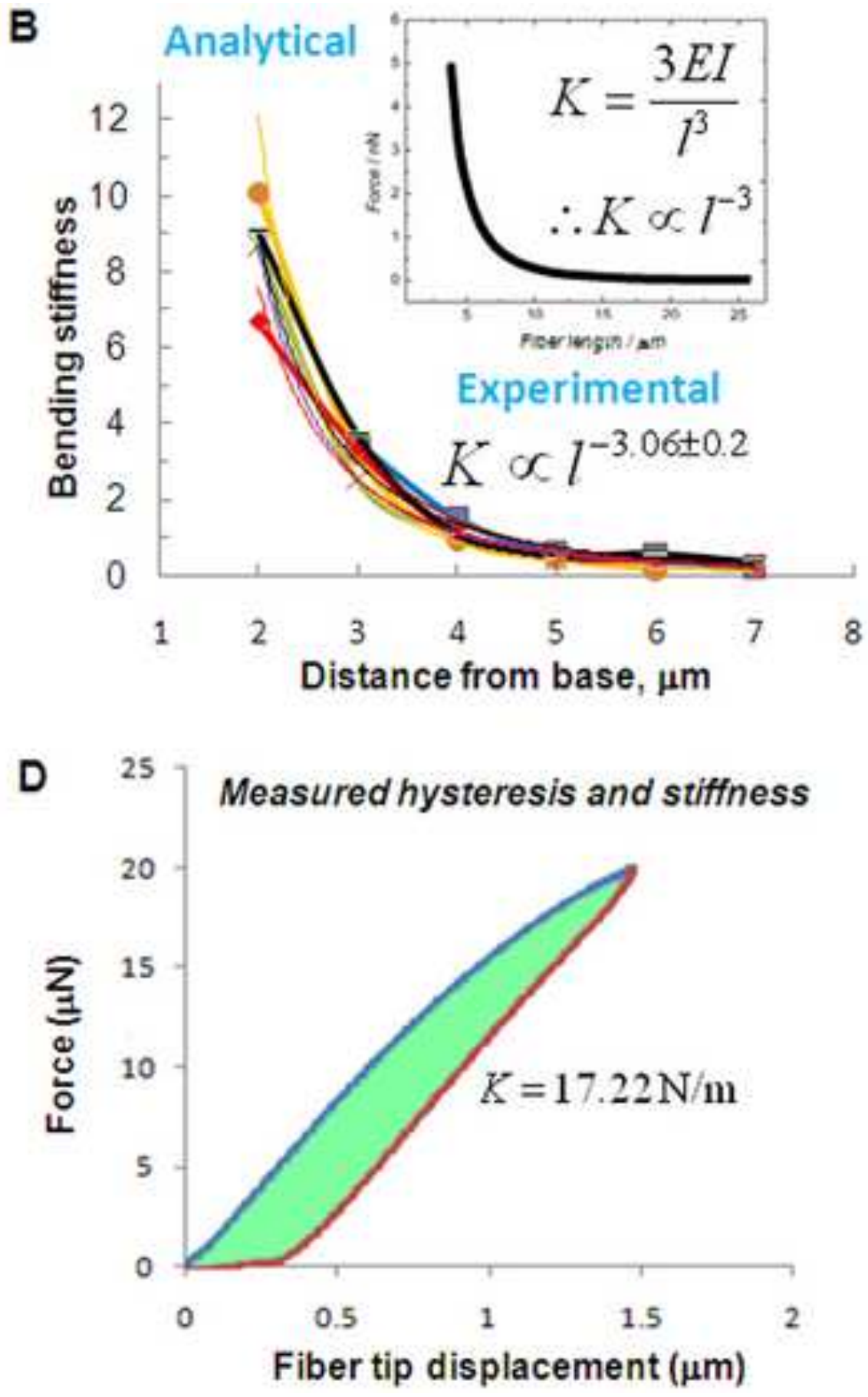
Figure 4.revised.jpg

Click here to download high resolution image
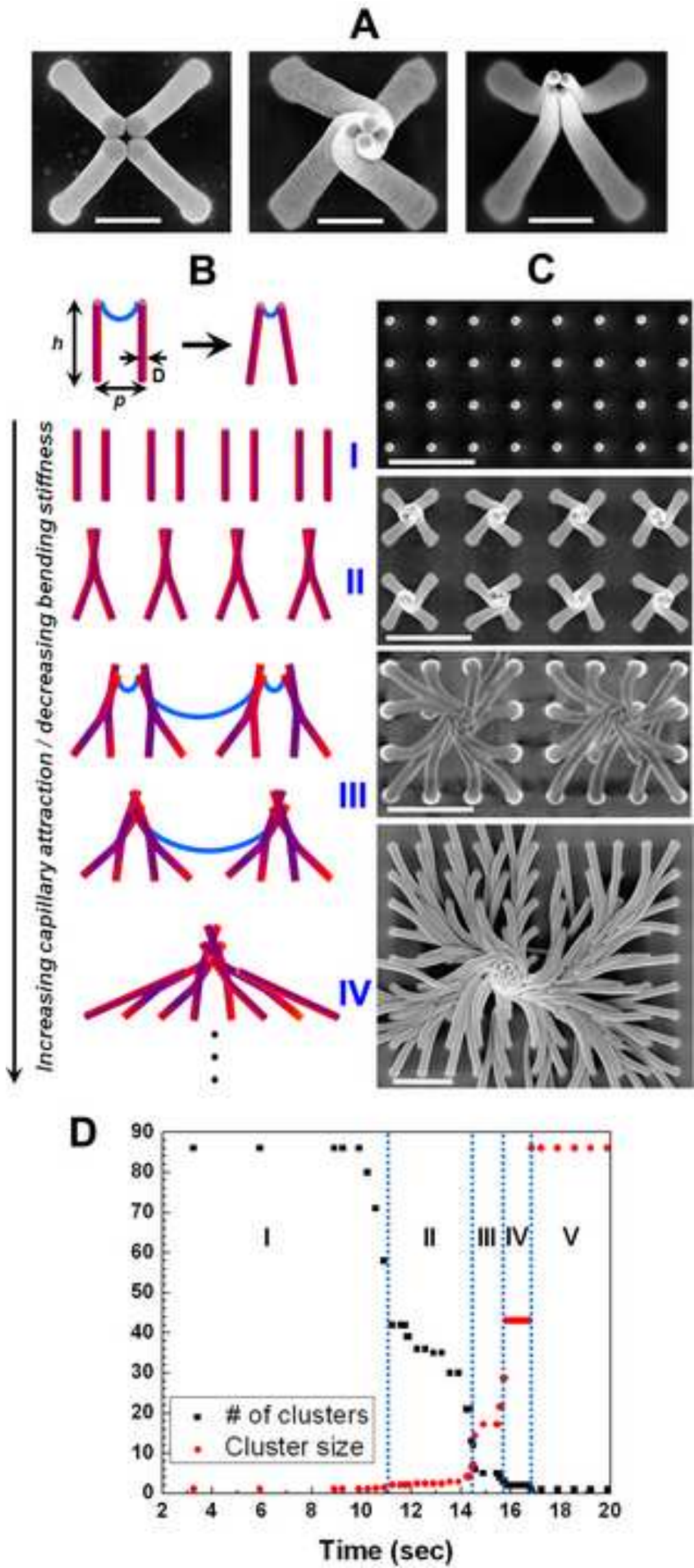


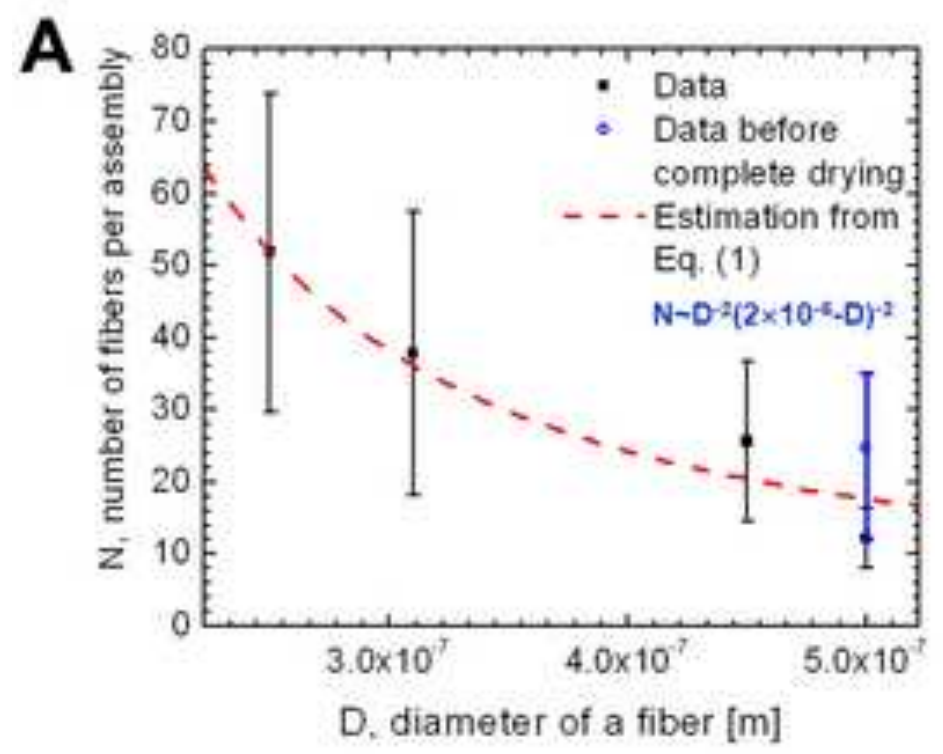

B

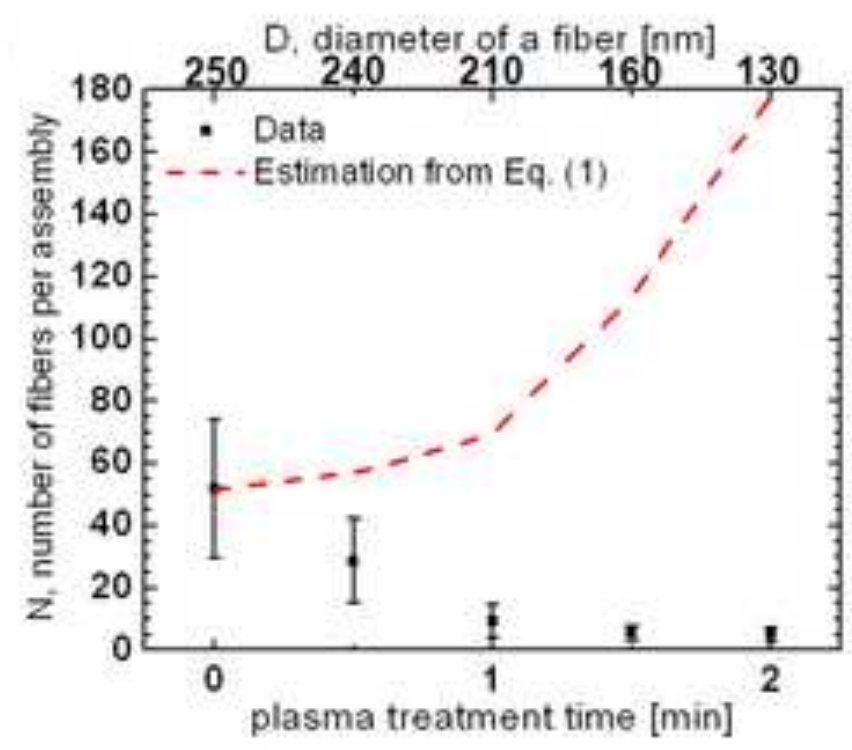

C
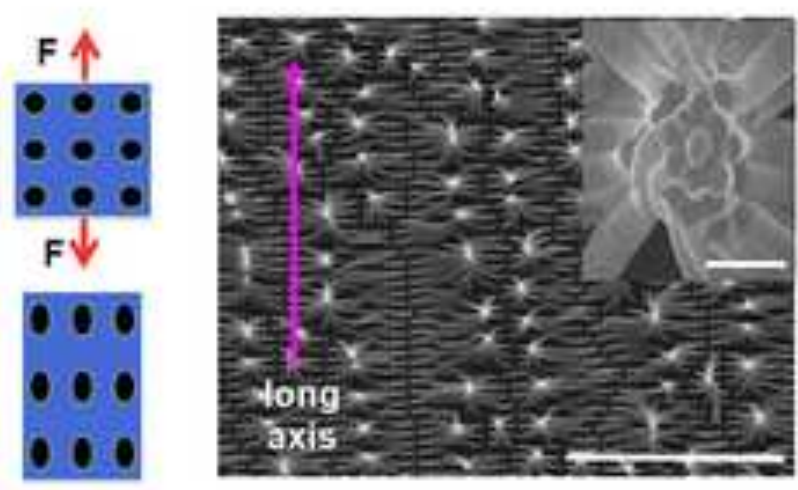

D

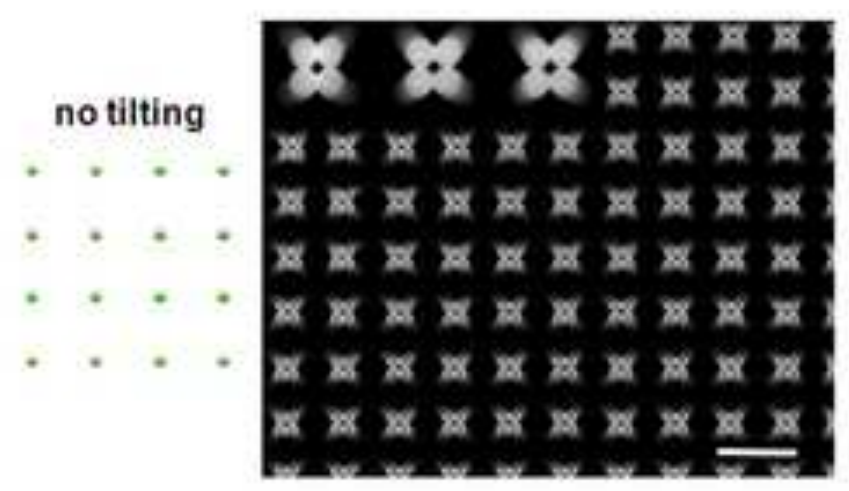

E

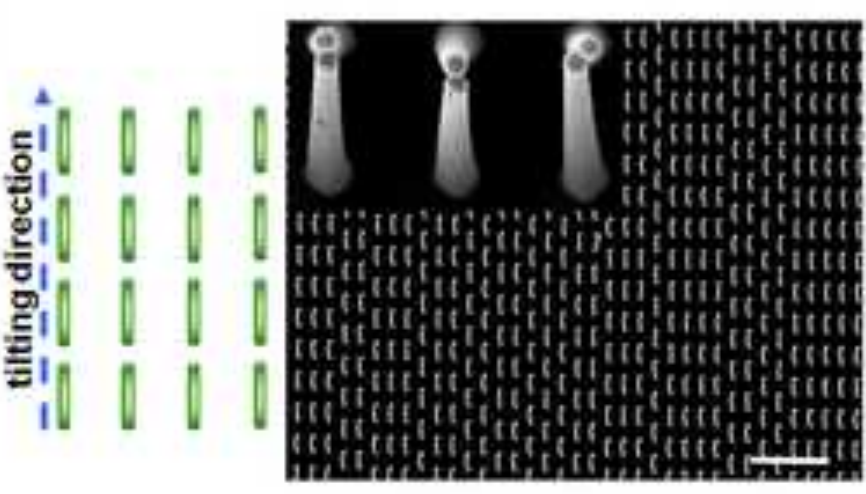

F

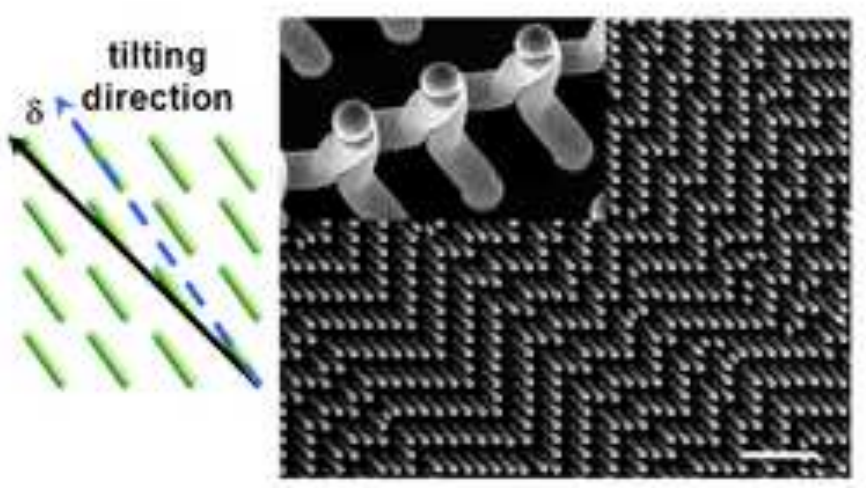


Figure 6.jpg

Click here to download high resolution image

A

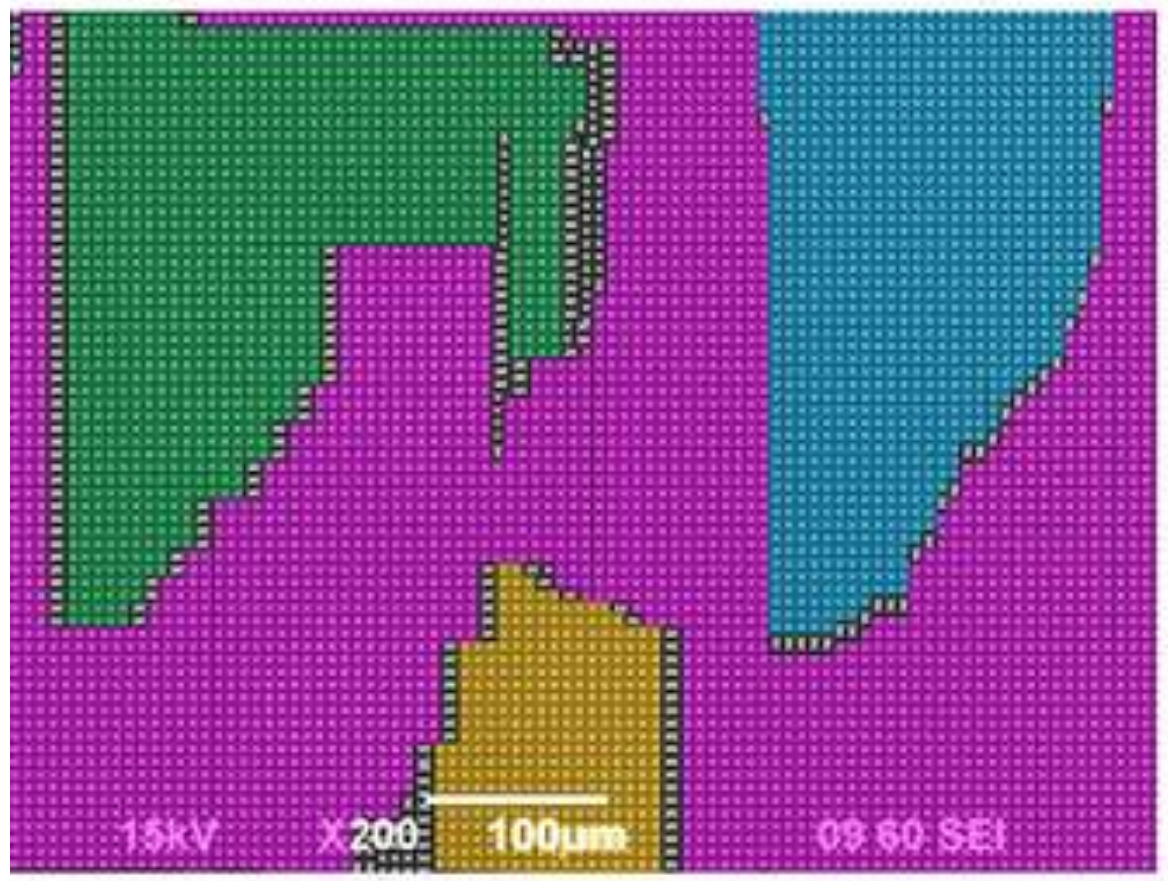

C

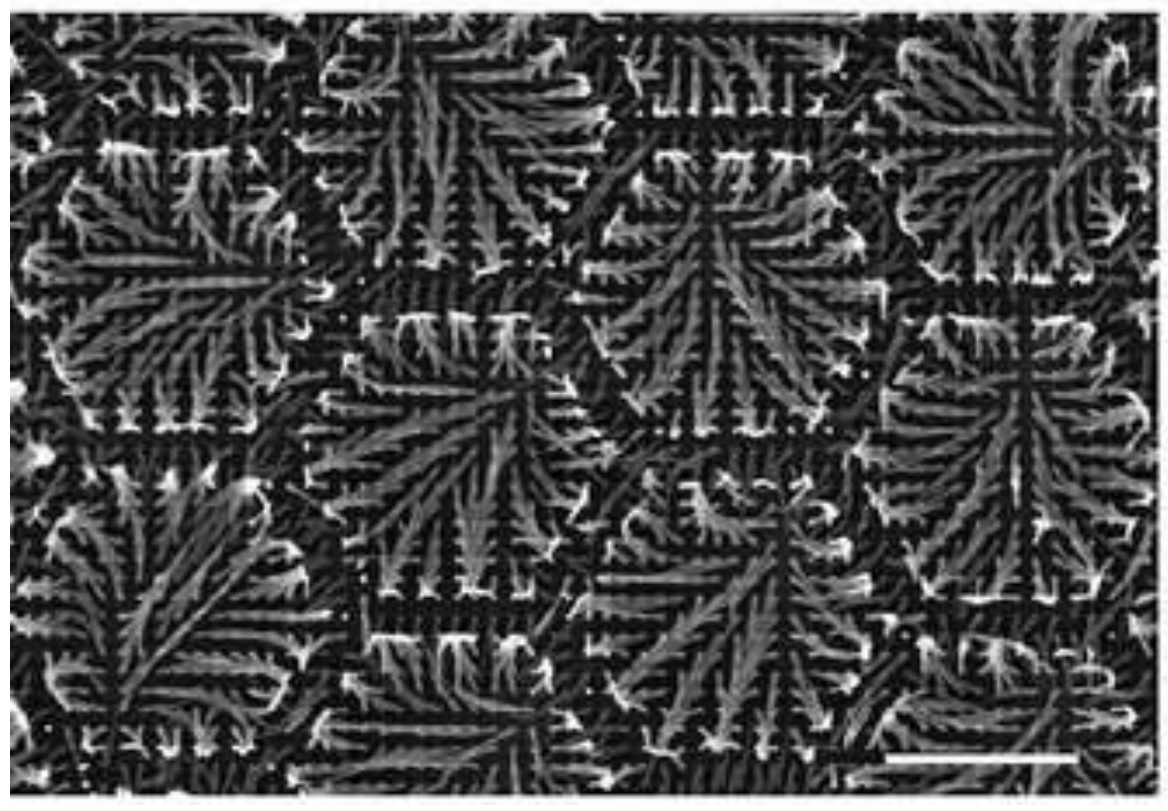

B

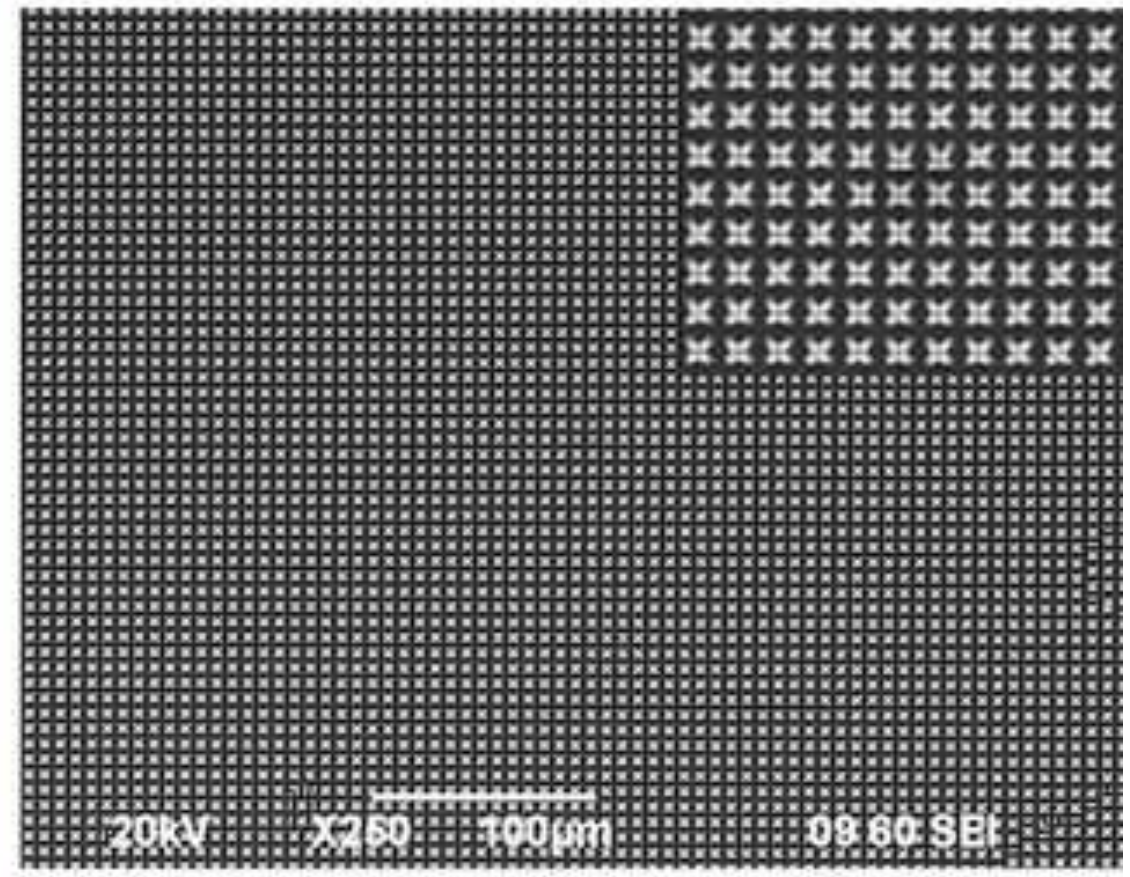

D

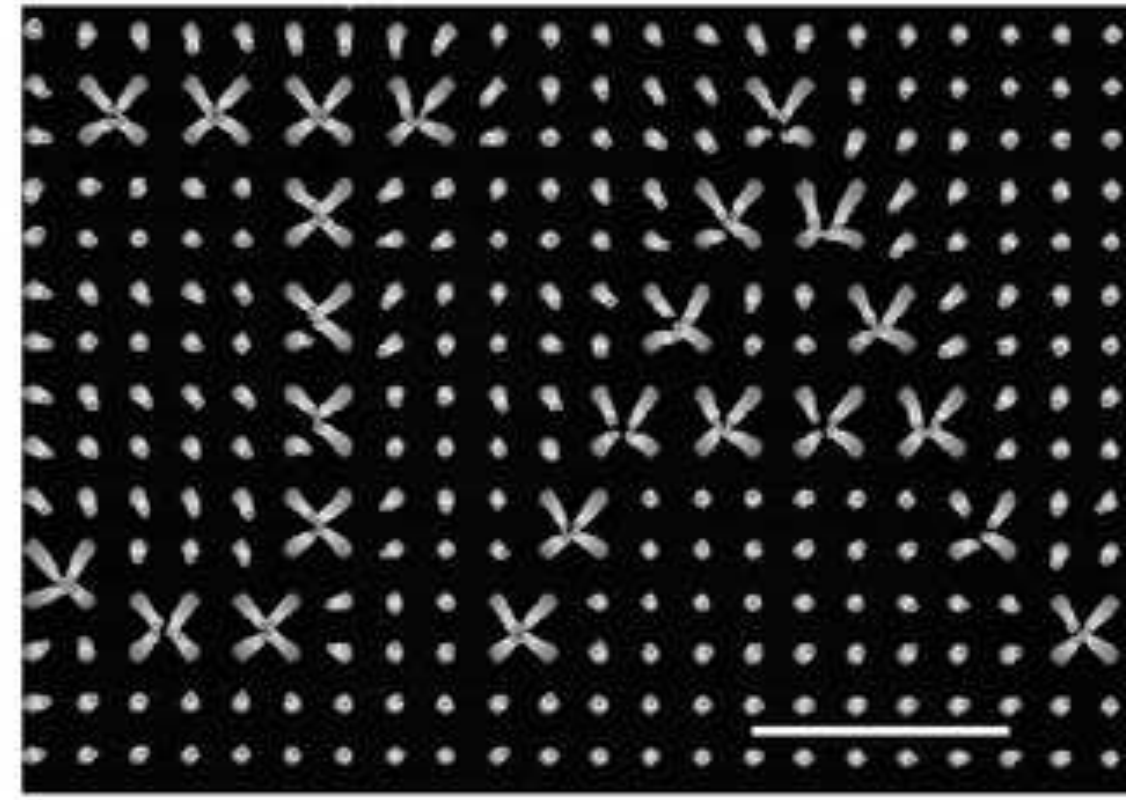




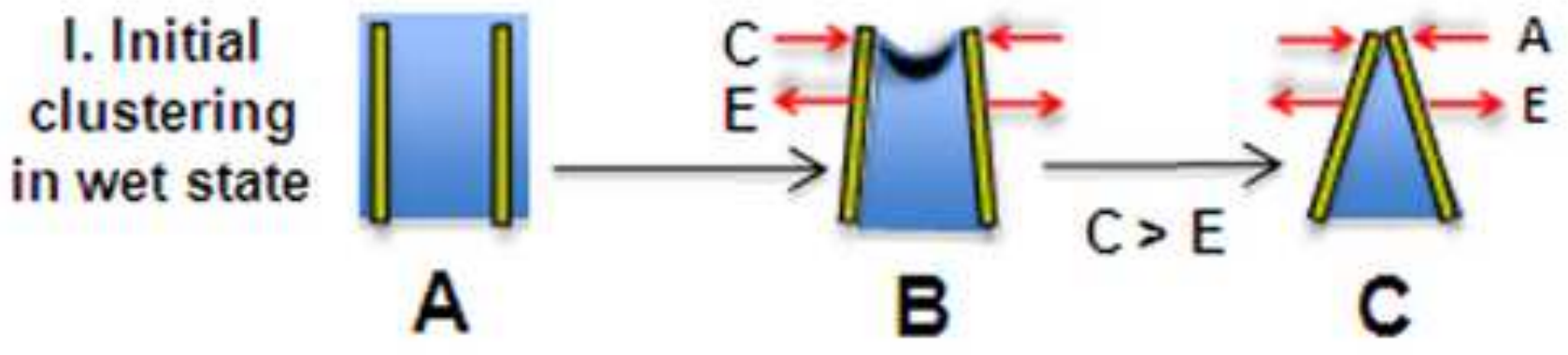

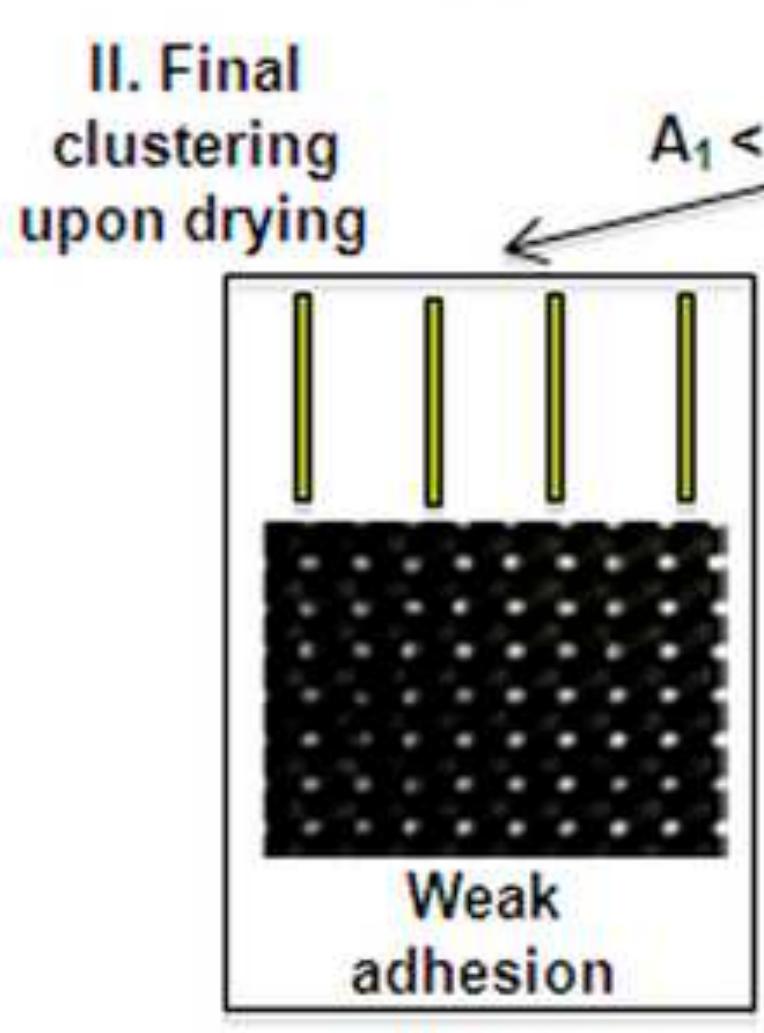

D

III. Inducing reversible assembly by changing chemical environment
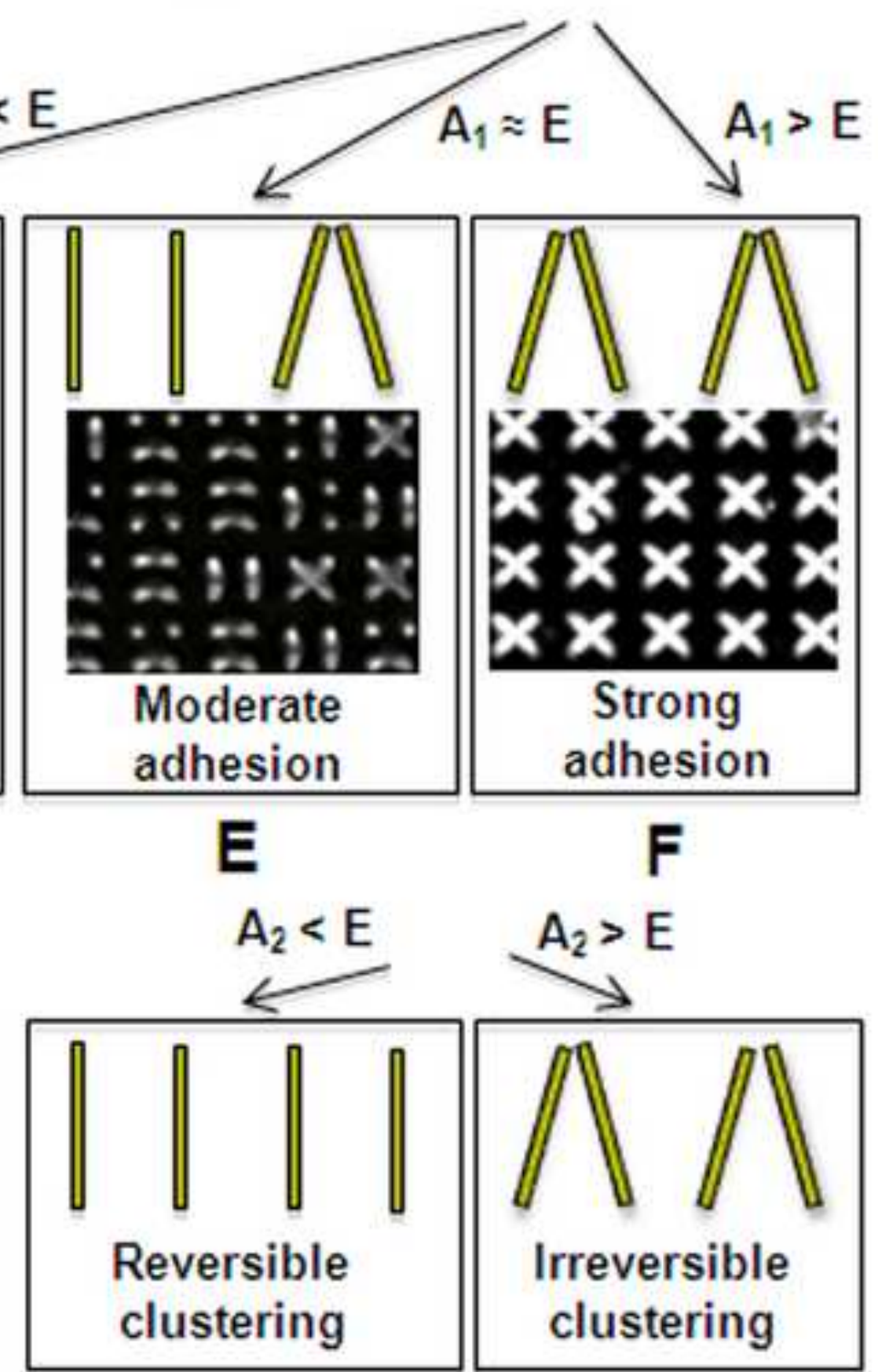

G 
A

$00: 00 ; 00 ; 120$,

$00 ; 00 ; 23 ; 03$

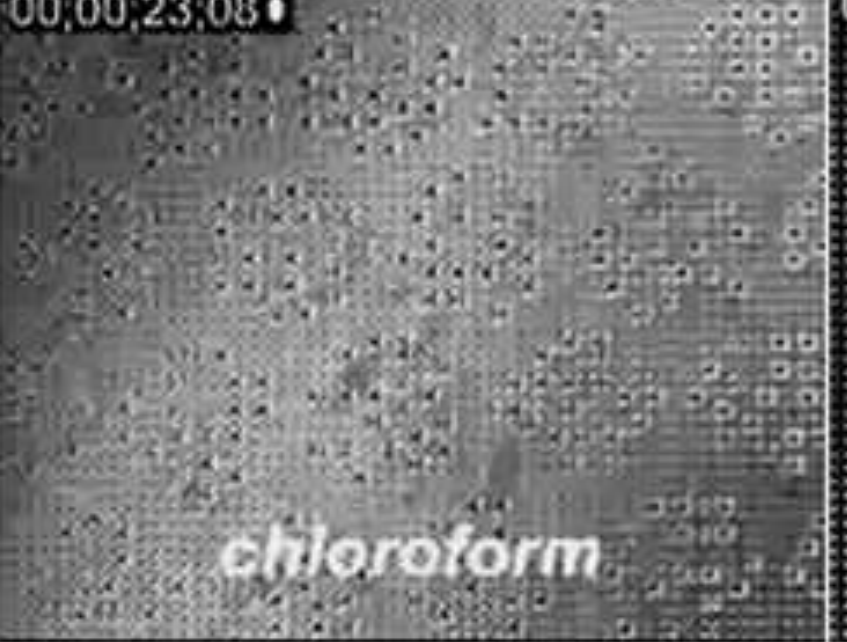

D
B

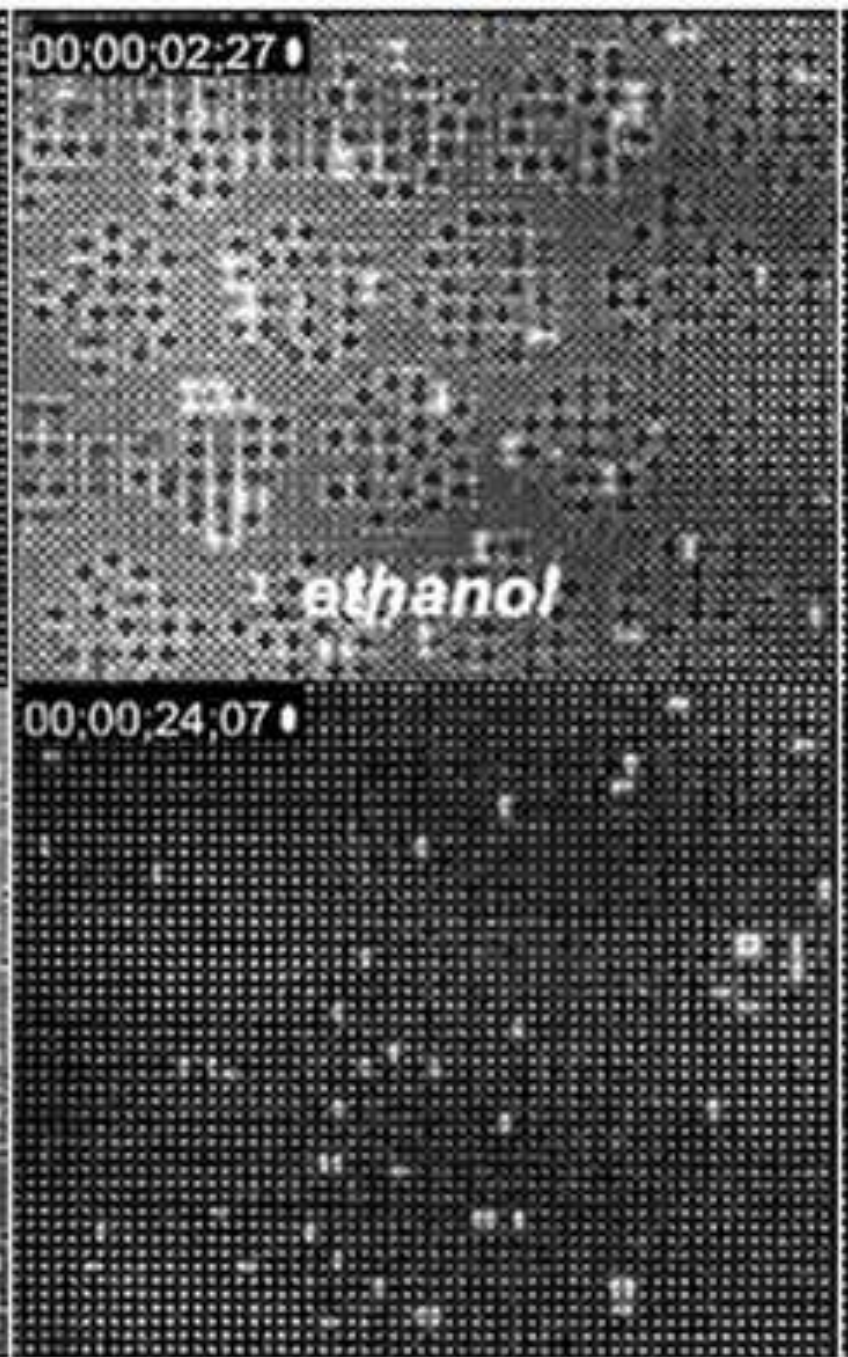

E
C

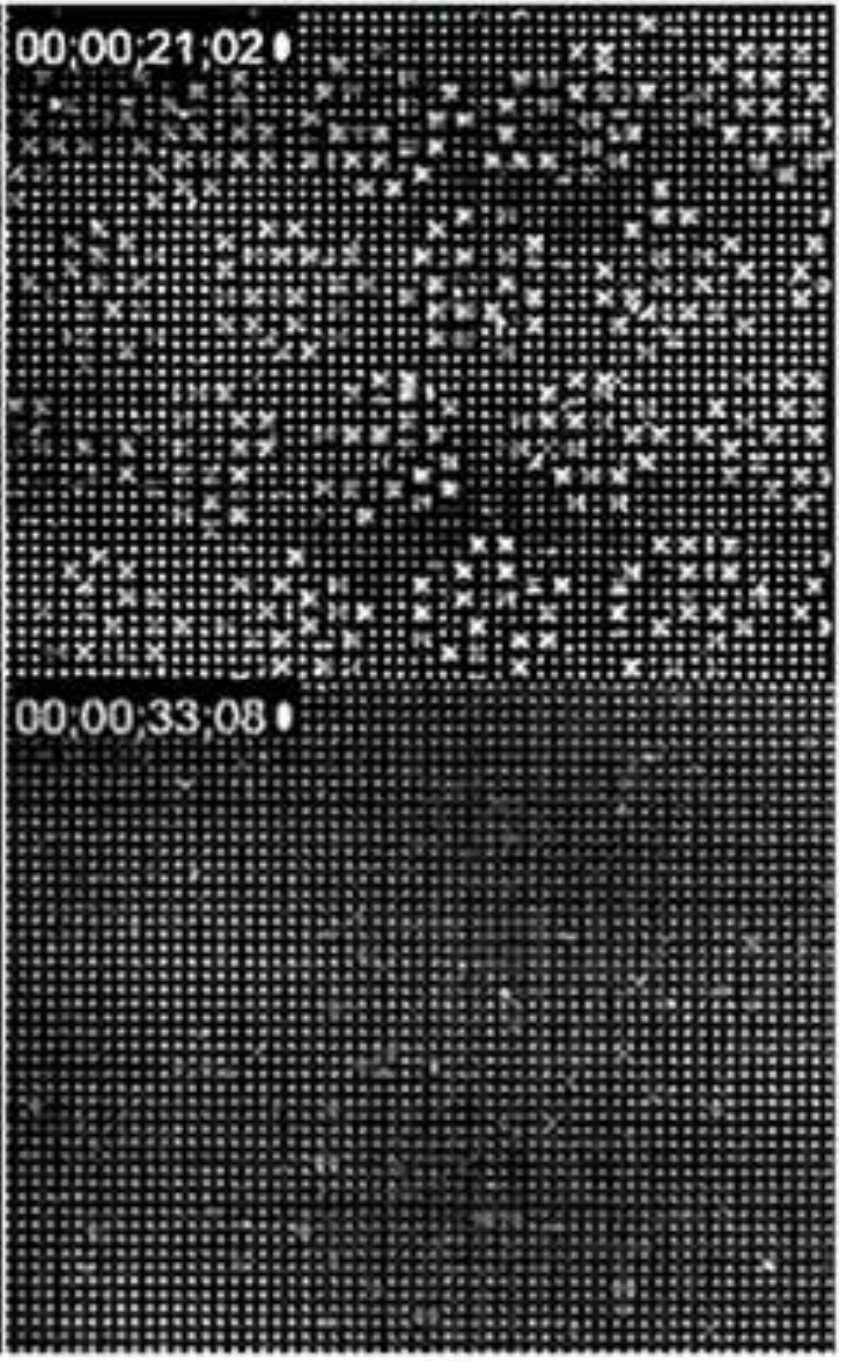

F 
Click here to download high resolution image

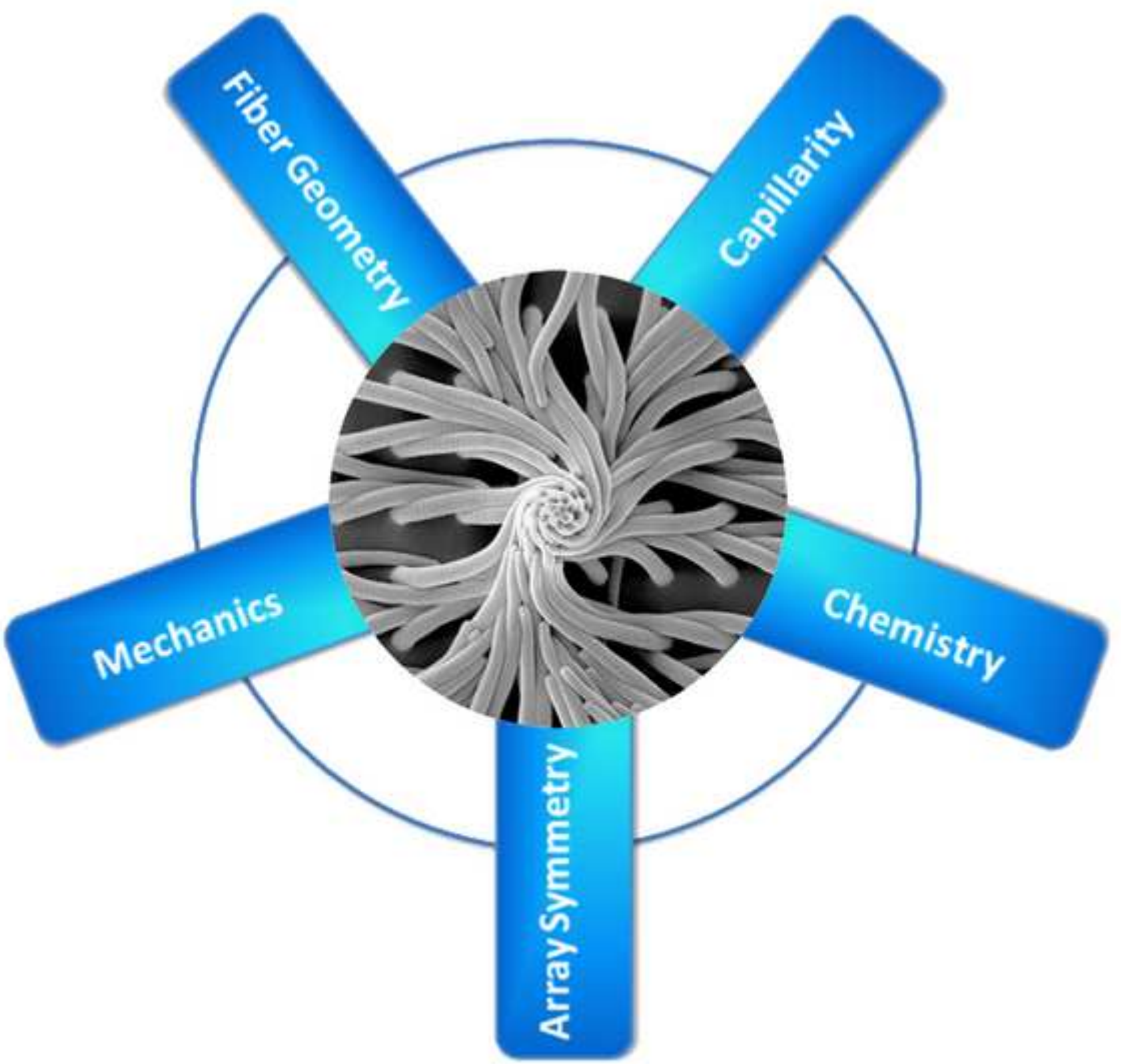




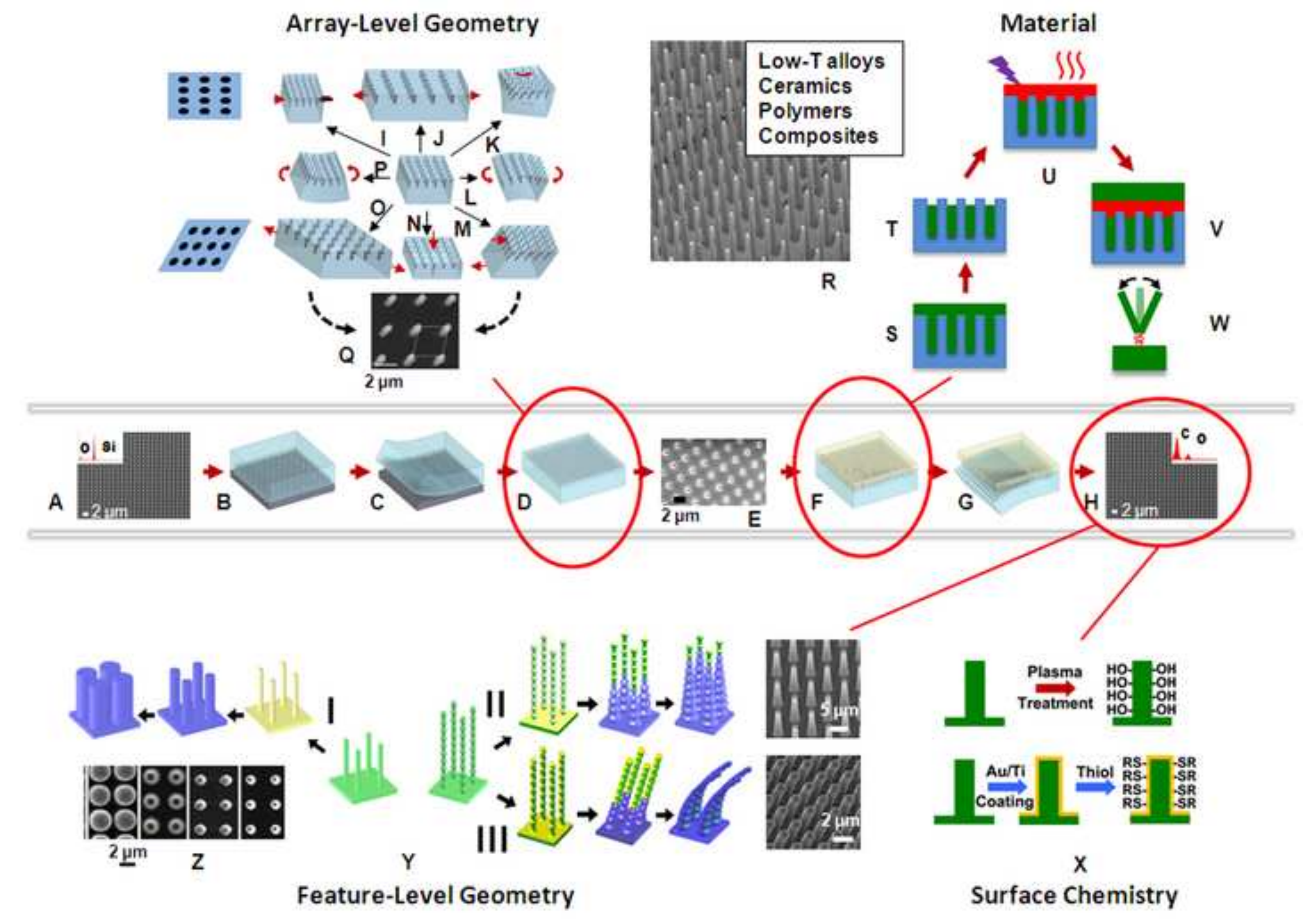


A

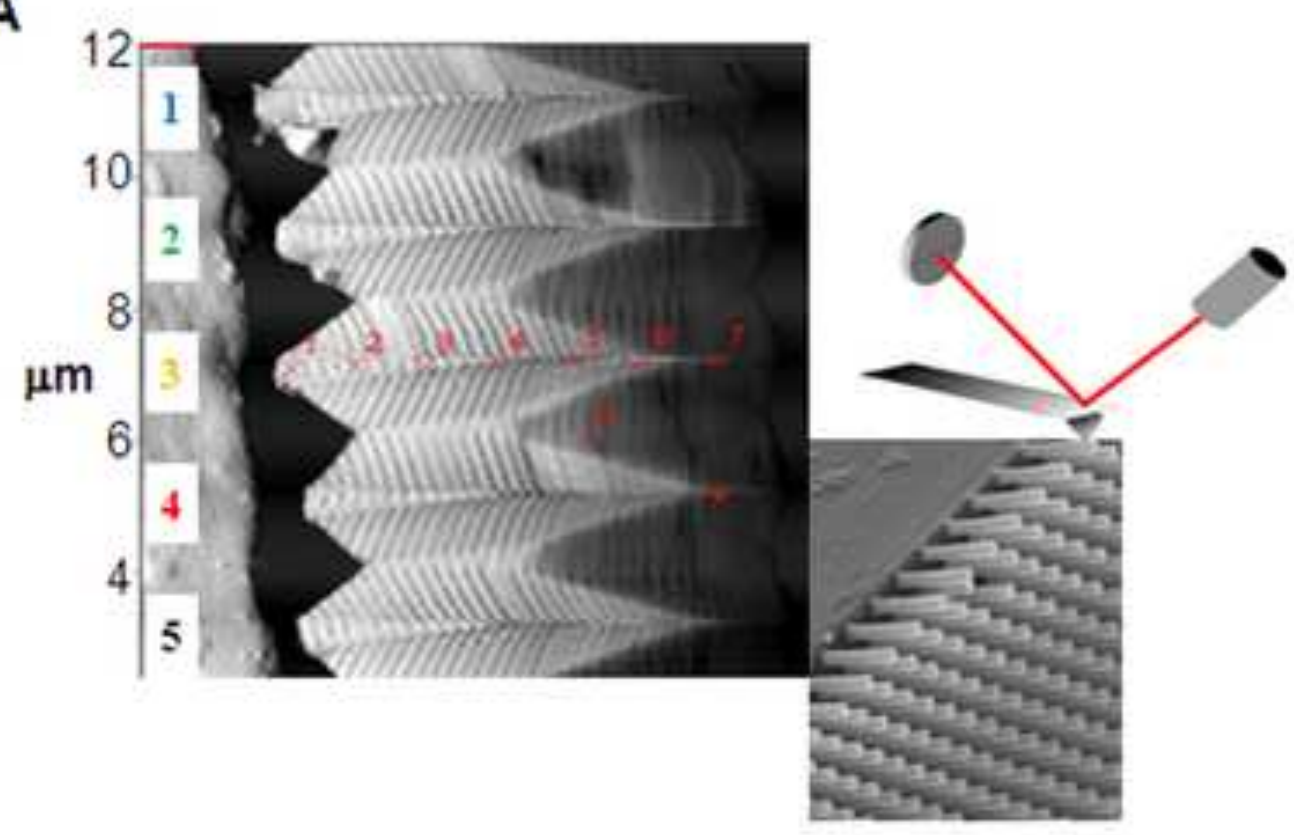

C

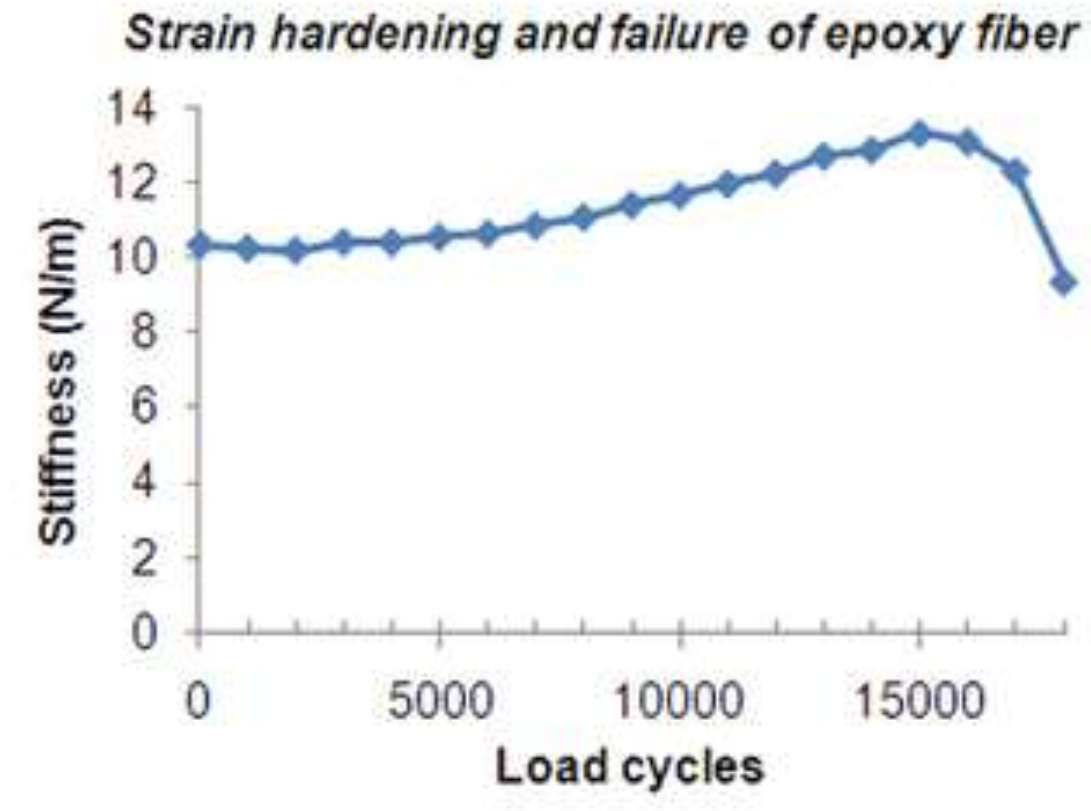

B
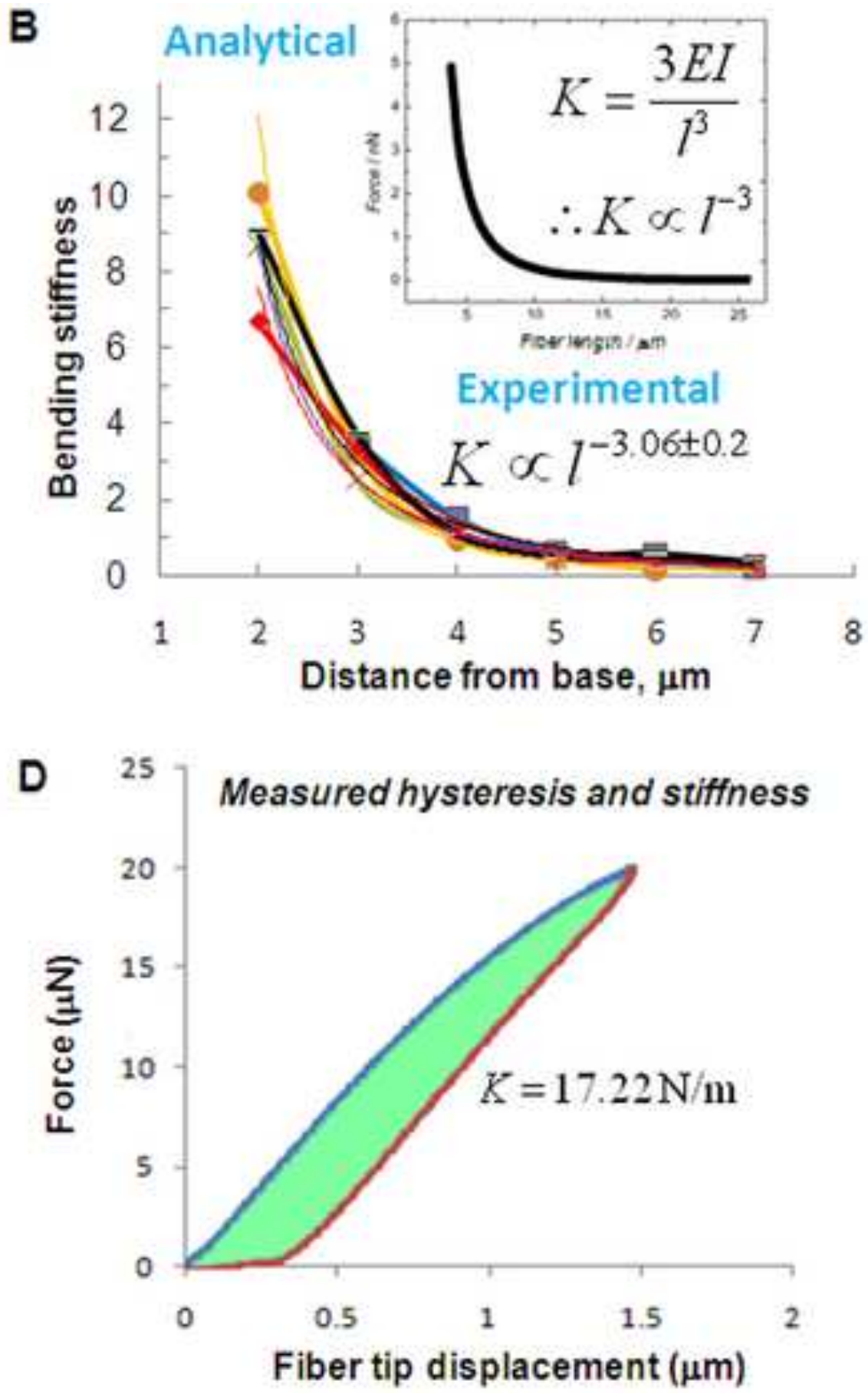
Figure4.revised.tiff

Click here to download high resolution image
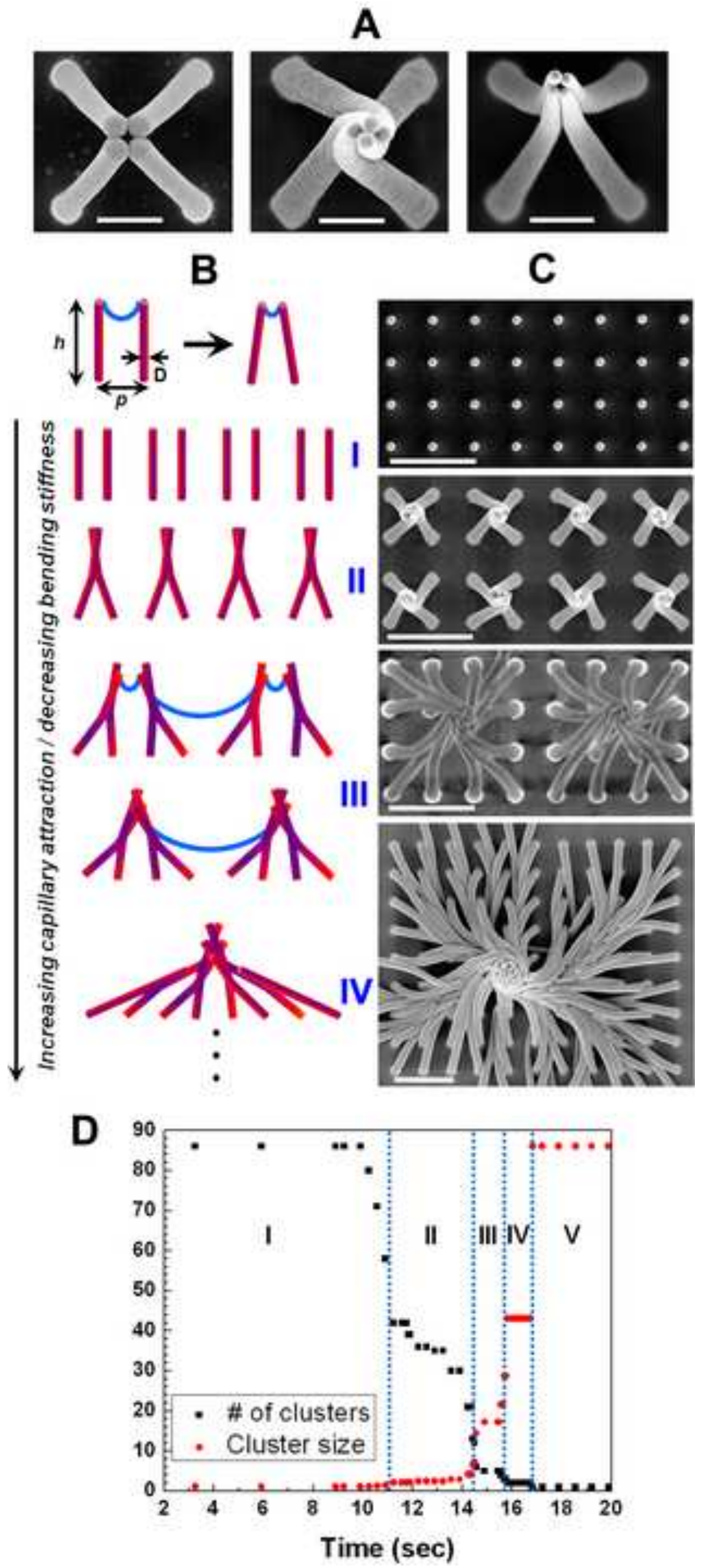


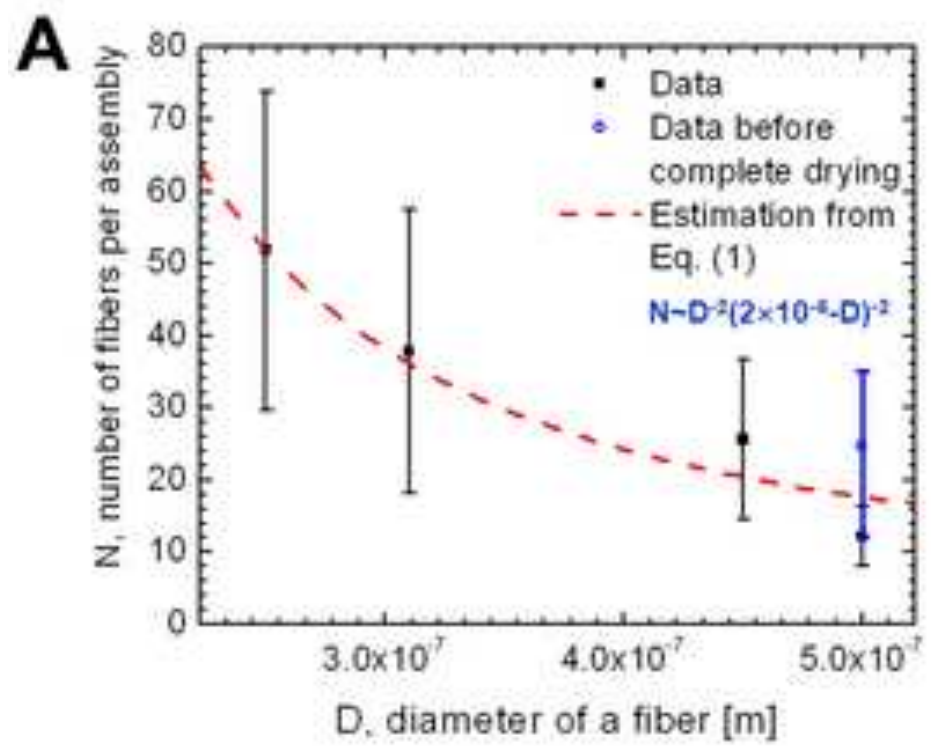

B

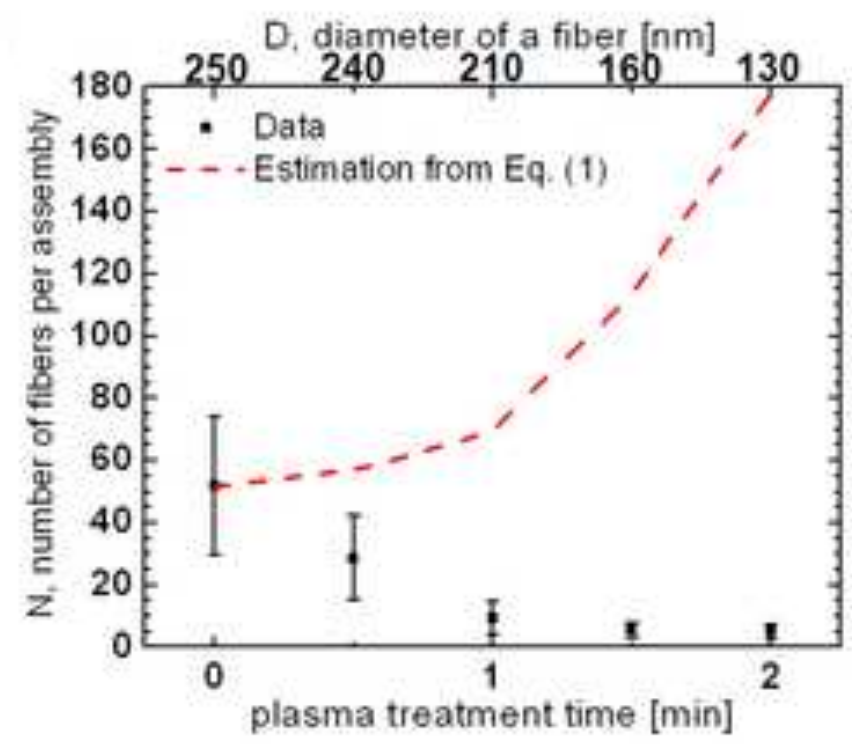

C
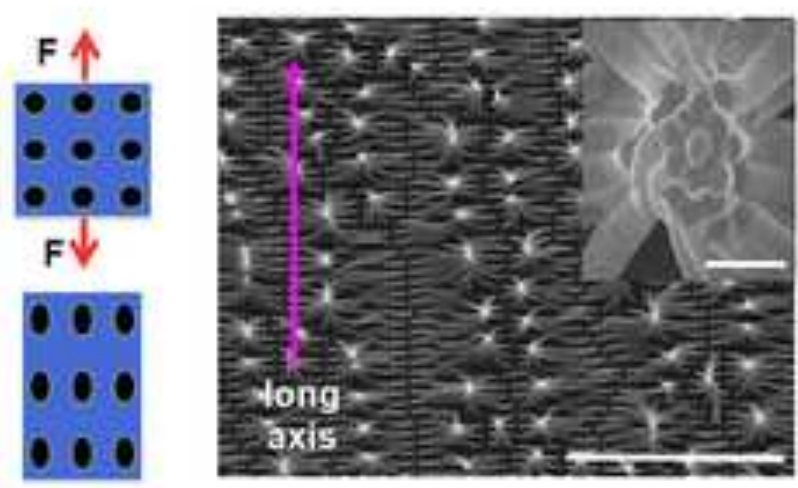

D

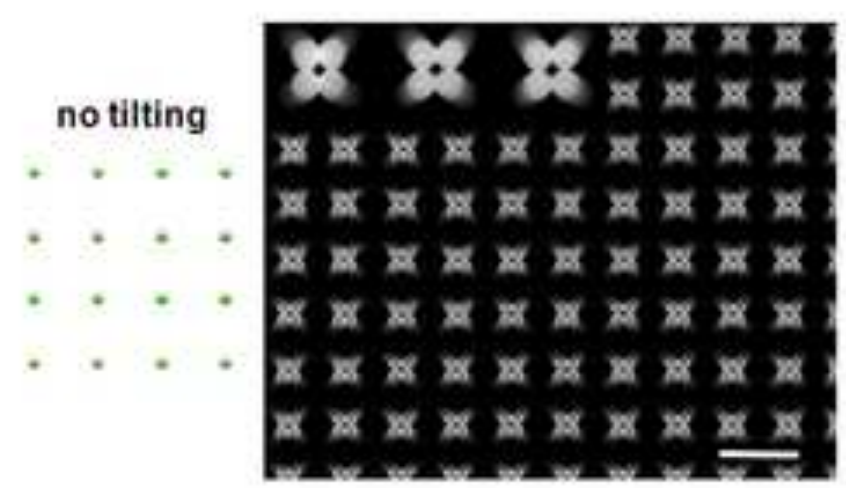

E

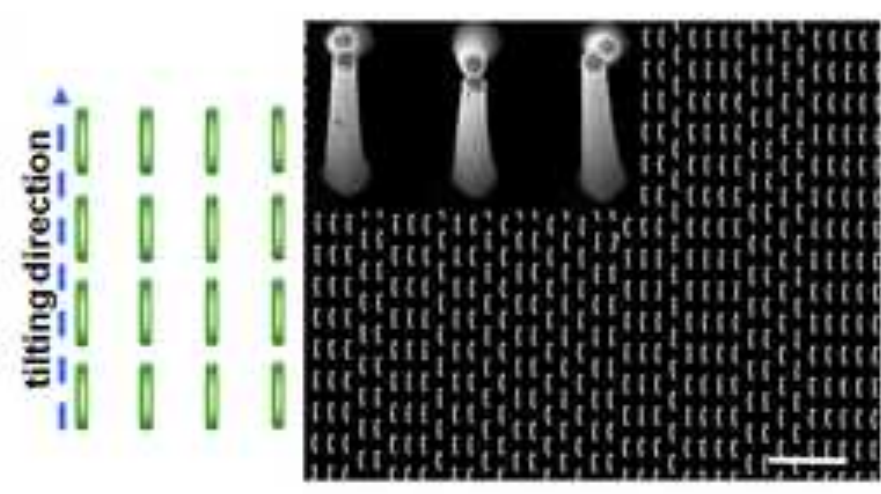

F

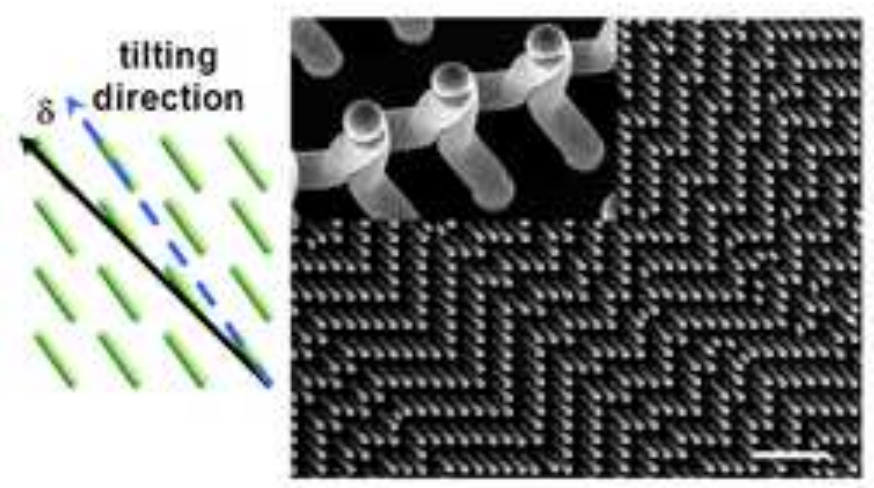


Figure6.tiff

Click here to download high resolution image

A

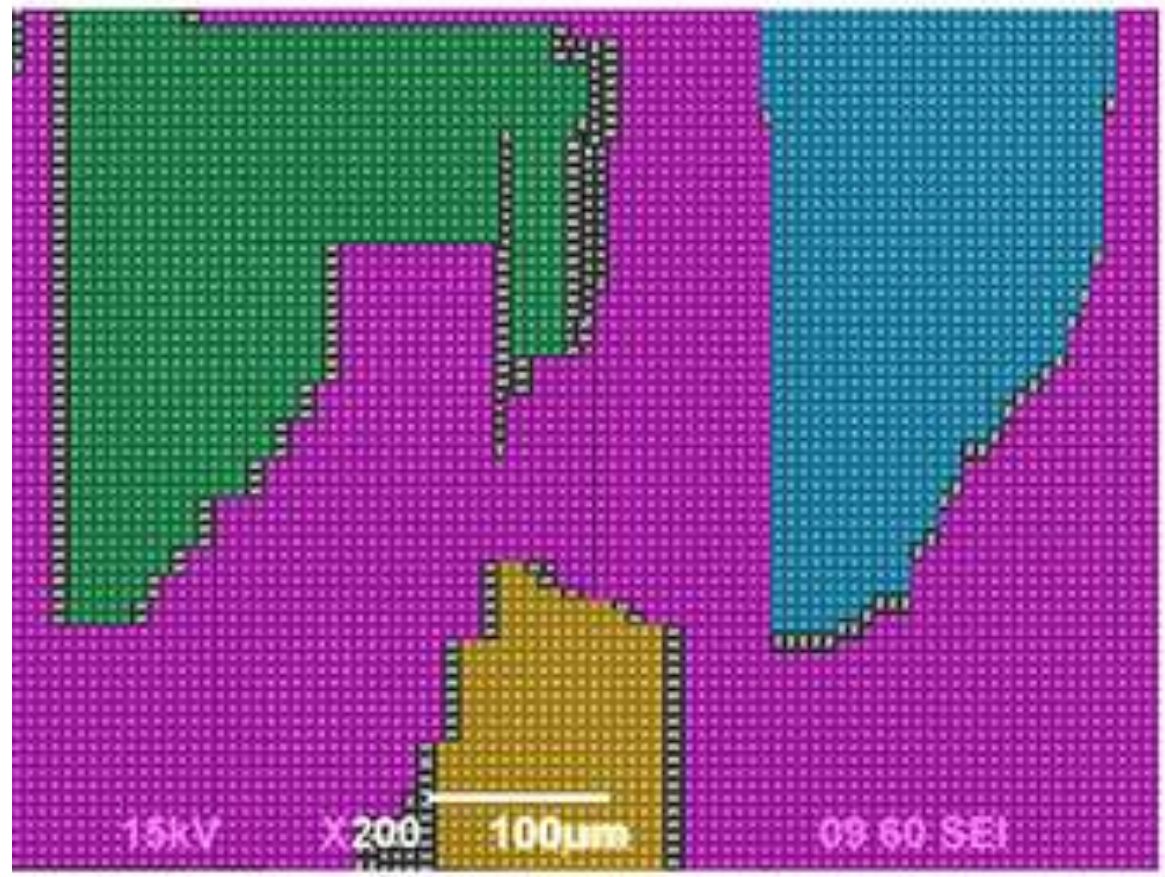

C

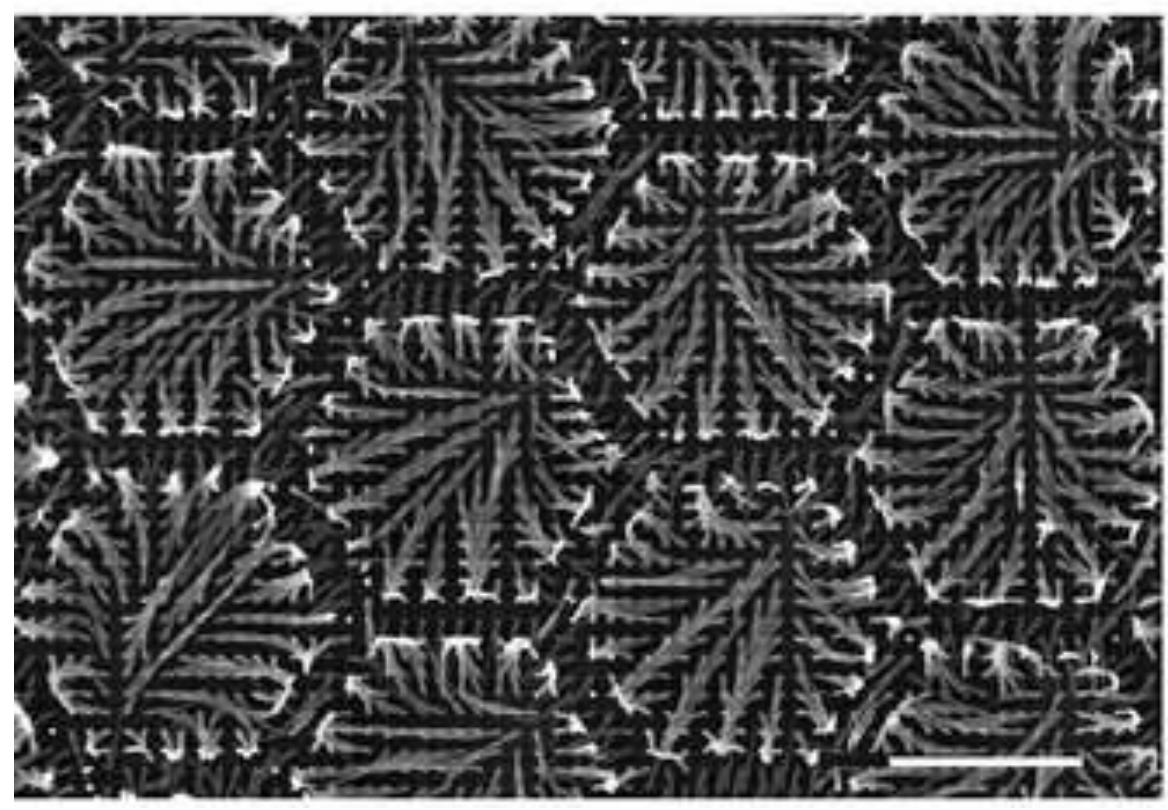

B

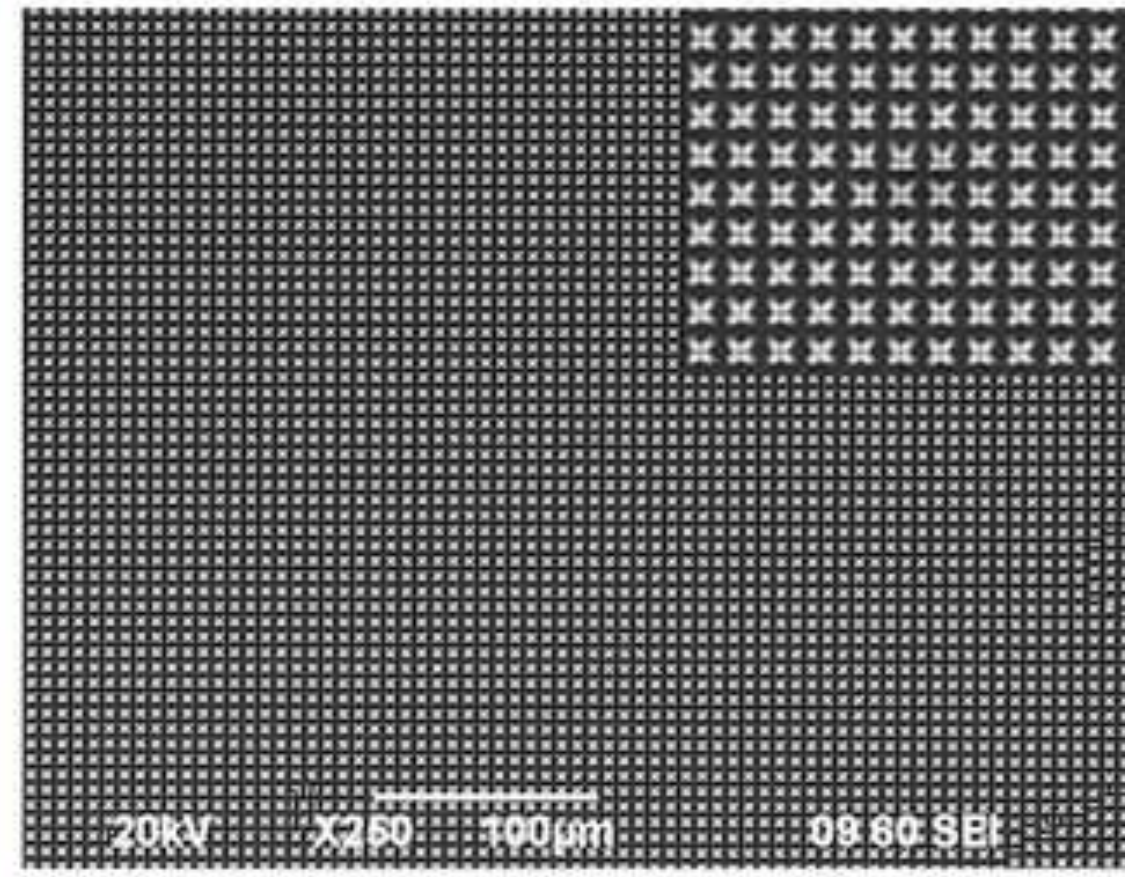

D

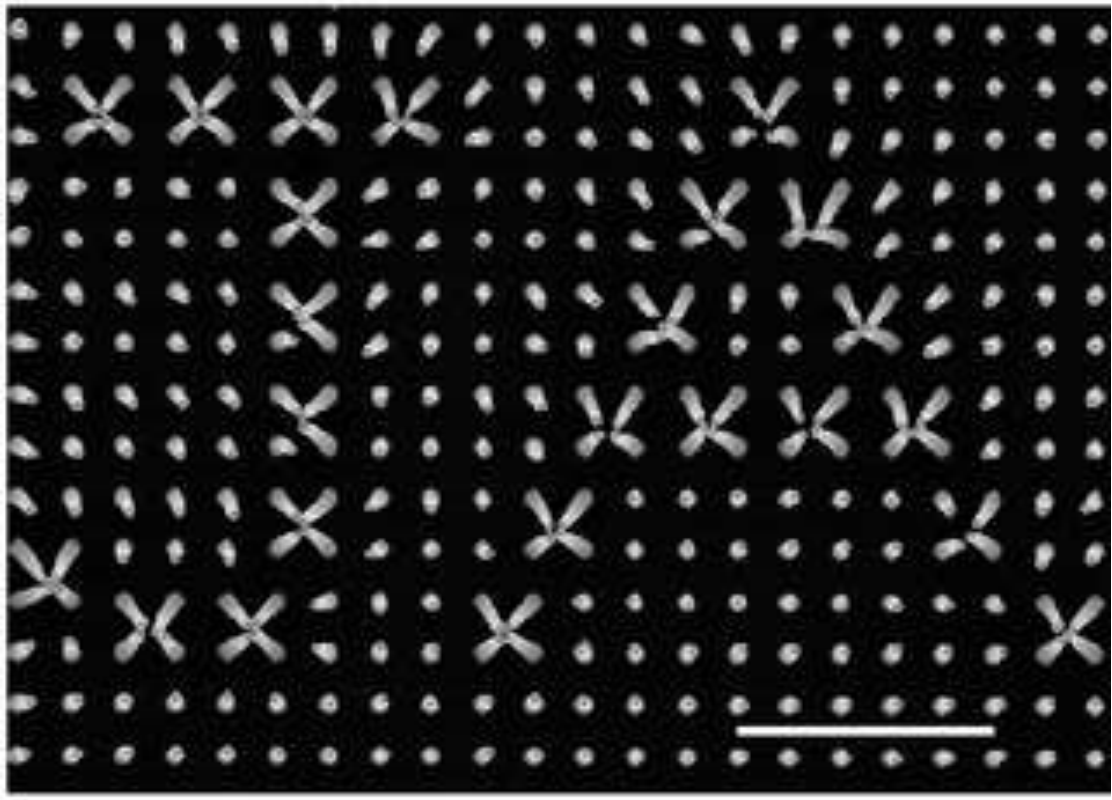




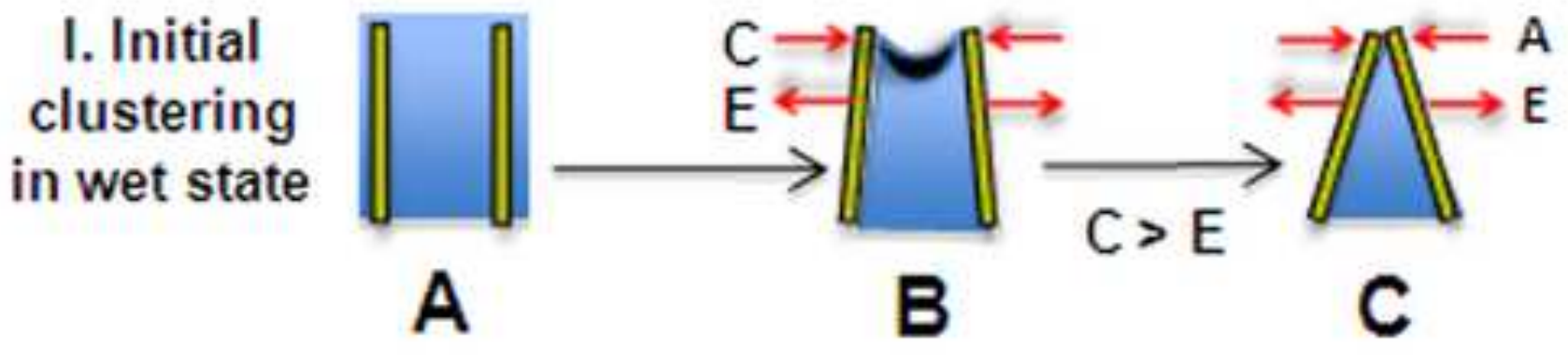

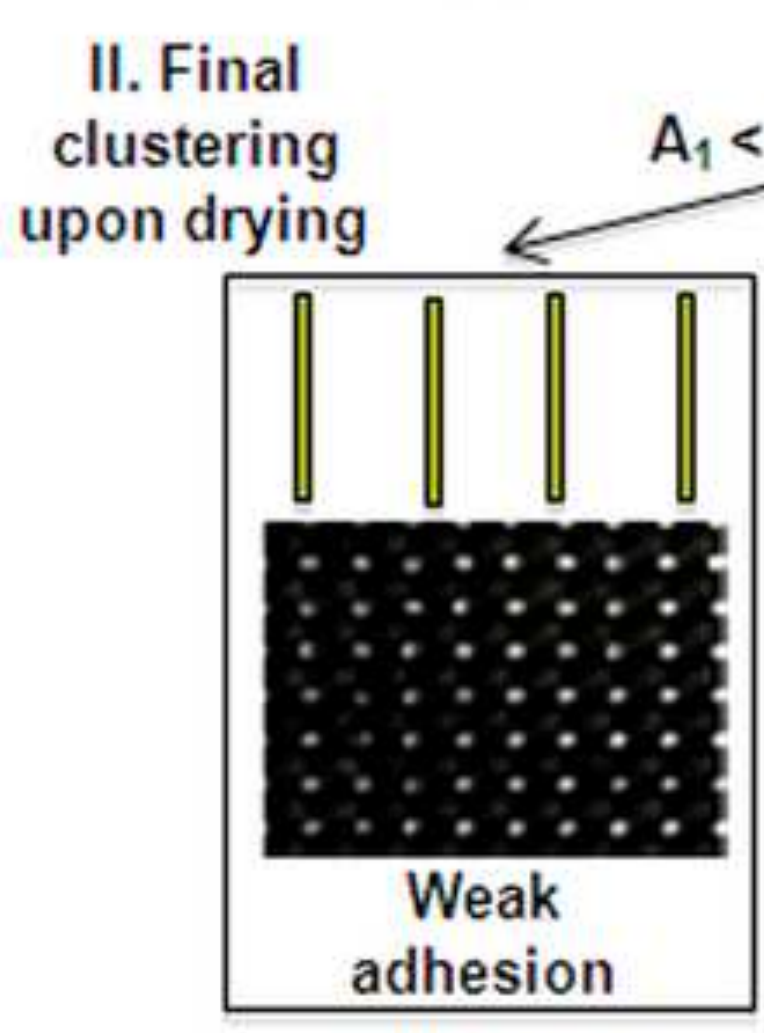

D

III. Inducing reversible assembly by changing chemical environment
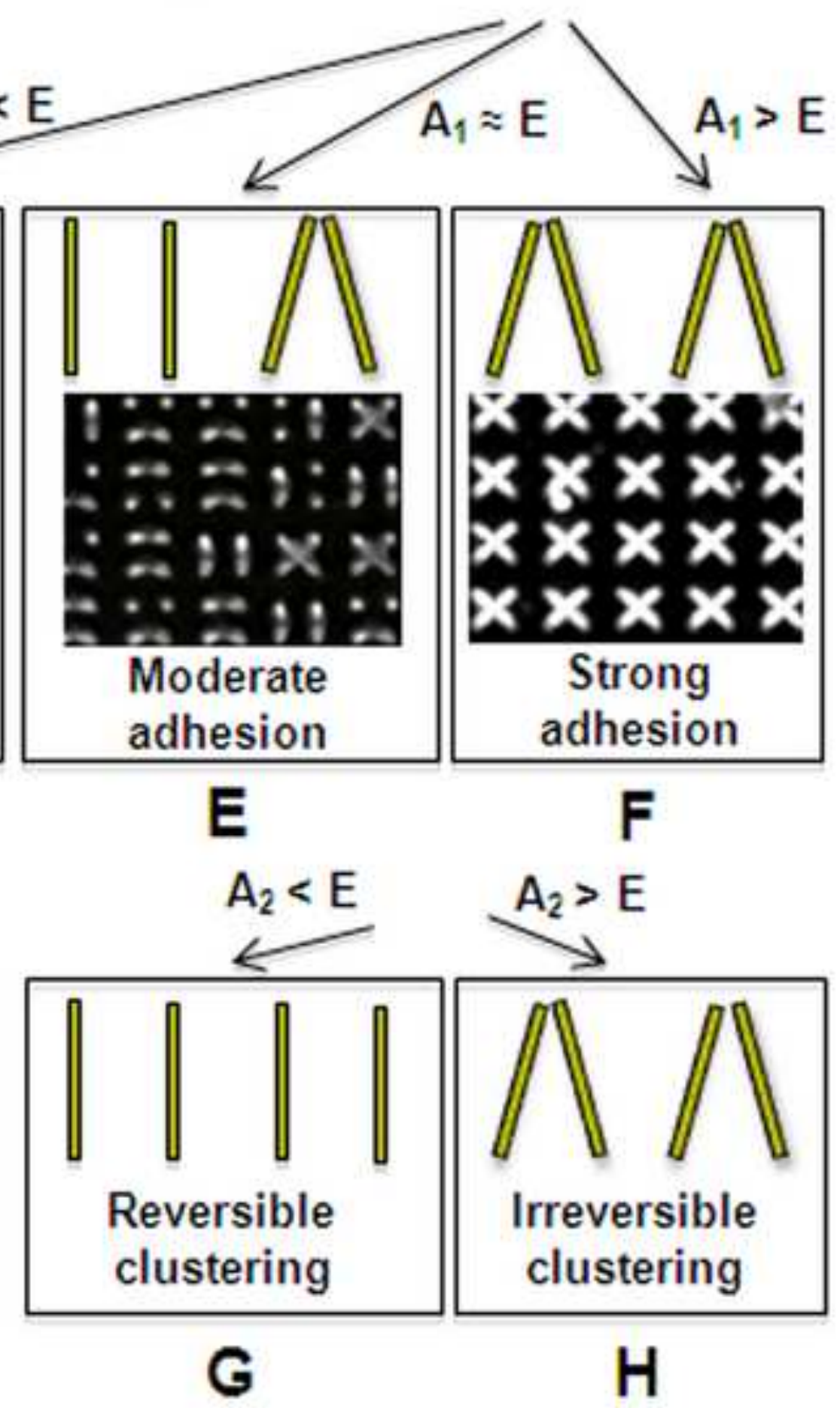
A

00,$00 ; 00 ; 120$,

$00 ; 00 ; 23 ; 08$ •

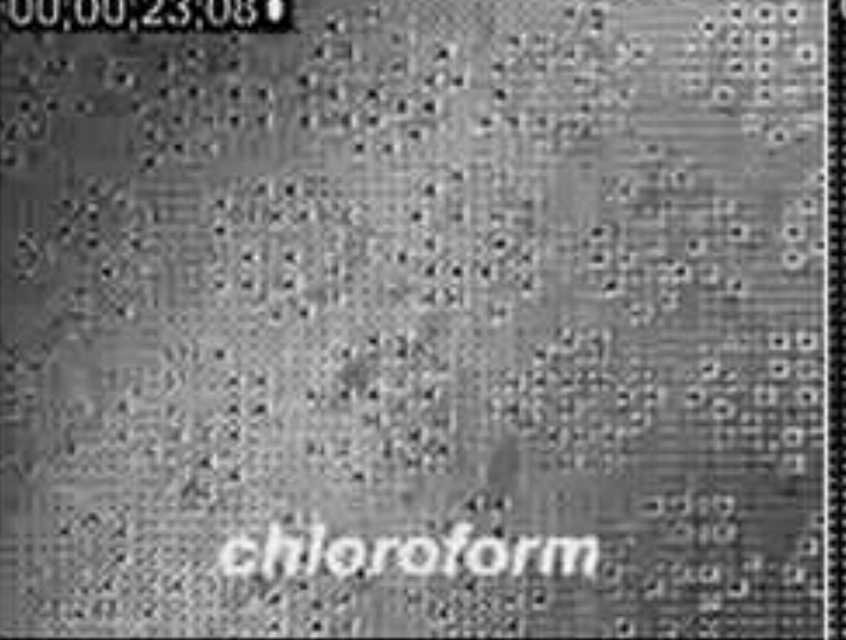

D
B

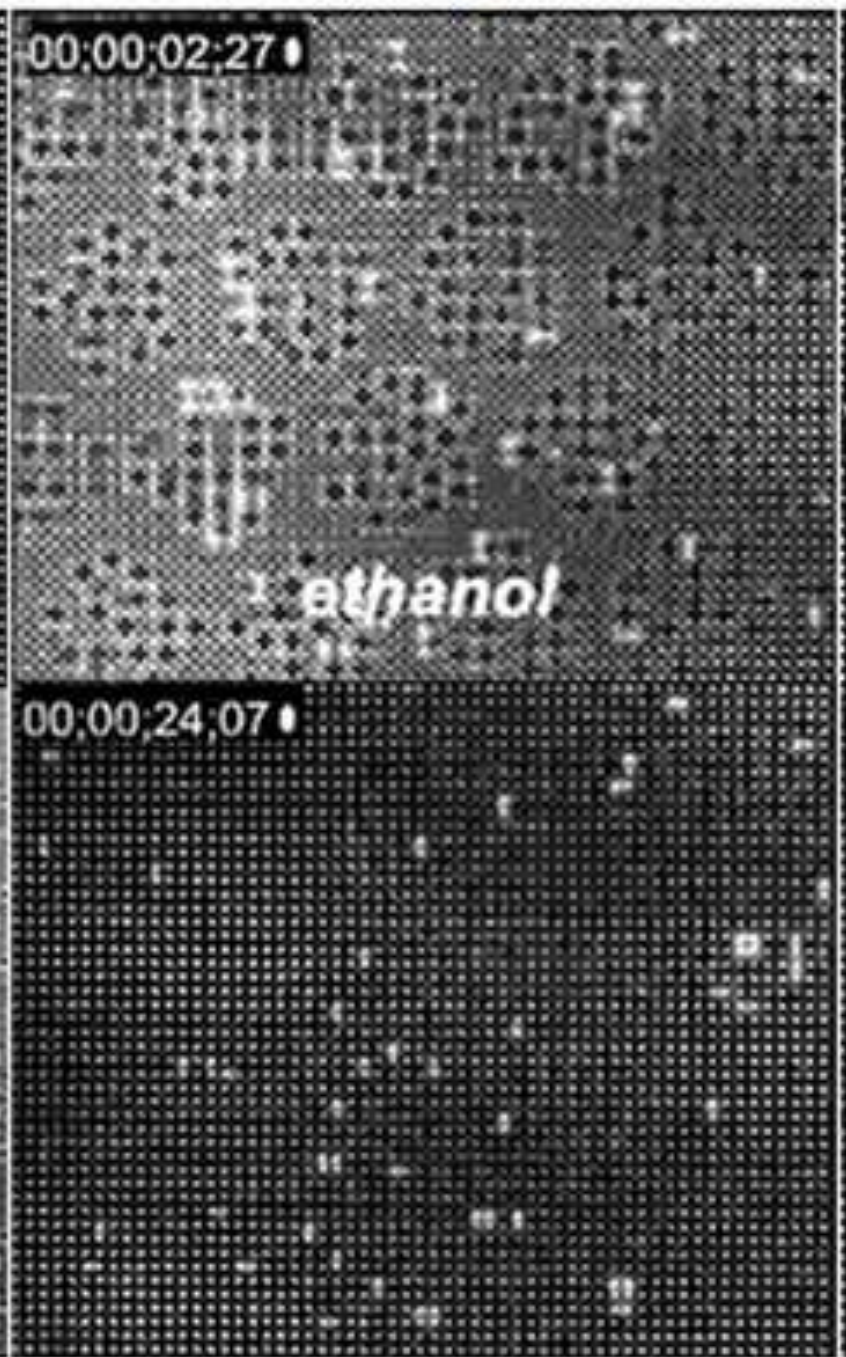

E
C

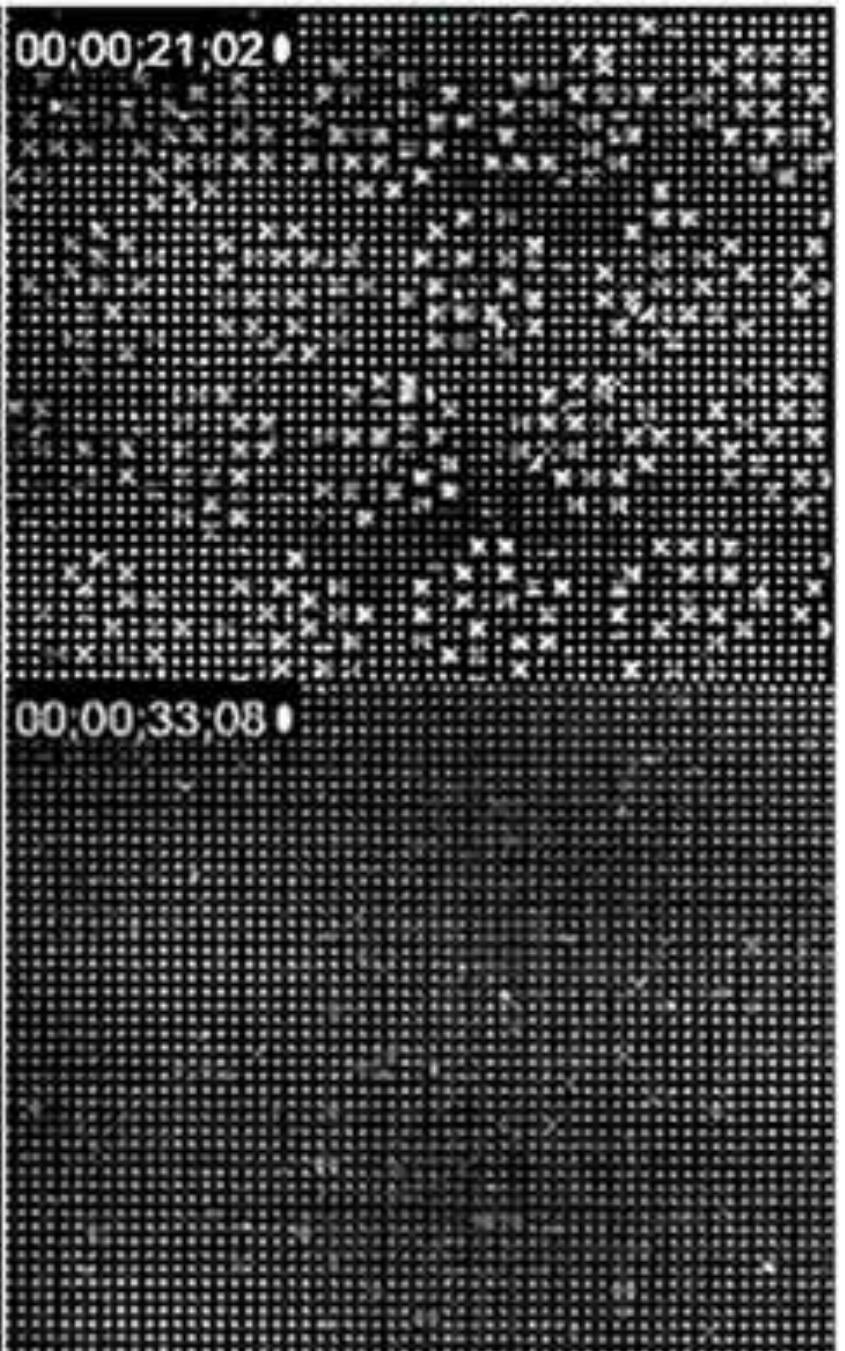

F 
Alison Grinthal is a Research Scientist in the School of Engineering and Applied Sciences at Harvard University, with interests spanning bioinspired engineering, dynamic and adaptive materials, self-assembly, surface and interfacial phenomena, structure and dynamics of macromolecules, and the interplay between chemistry and mechanics in biological and synthetic systems. She received her B.A. from Swarthmore College in both chemistry and anthropology, and received her Ph.D. from Harvard University in biochemistry. Her graduate and postdoctoral work explored how the fluctuations of membrane-embedded proteins translate lipid bilayer mechanical properties into enzymatic activity, and how atomic interactions at helix-helix interfaces collectively give rise to larger scale protein elasticity.

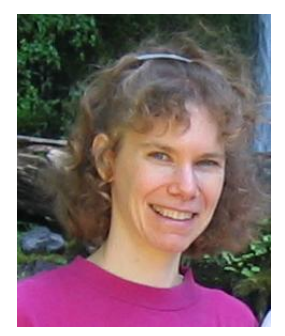

Sung H. Kang is pursuing a Ph.D. degree in Applied Physics at Harvard University under the supervision of Professor Joanna Aizenberg. He is interested in the interaction of micro/nanostructures with fluids. His current research focuses on the self-assembly of nanofibers in an evaporating liquid and on surface wetting/dewetting phenomena. In particular, he studies how chiral structures can arise from achiral starting materials. Before beginning the Ph.D. program, he worked as a staff scientist at EIC Laboratories, Inc. Prior to his industry experience, he earned his master's degree in Electronic Materials from MIT, following his bachelor's degree in Materials Science and Engineering from Seoul National University, Korea.

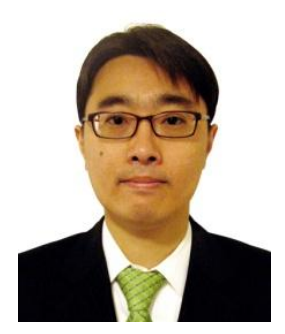

Alexander K Epstein is a Ph.D. Candidate in Engineering Sciences at the Harvard University School of Engineering and Applied Sciences, where his current research focus is in designing and studying nanostructured, active surfaces for applications including biofouling and adhesion control. Previously he received an S.M in Applied Physics from Harvard and a B.S. in Engineering from the Franklin W. Olin College of Engineering. Epstein's prior research interests 
include the structure-property relationships of mollusk nacre as well as system integration of an autonomous vehicle for the 2007 DARPA Urban Challenge.

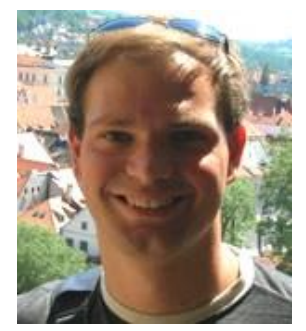

Michael Aizenberg received his Ph.D. in Organometallic Chemistry from The Weizmann Institute of Science in 1996 where he studied metal-mediated and catalyzed bond activation processes. Following a post-doctoral stay at MIT, he spent more than a decade working in process research and chemical development for flavor and fragrance products at Firmenich and for pipeline drug candidates at Vertex Pharmaceuticals before joining Wyss Institute for Biologically Inspired Engineering in 2007 as a Senior Staff Scientist. Aizenberg's current research interests are in designing active, adaptive, and programmable materials and drugdelivery systems, in tissue engineering, and in developing energy-efficient materials and devices.

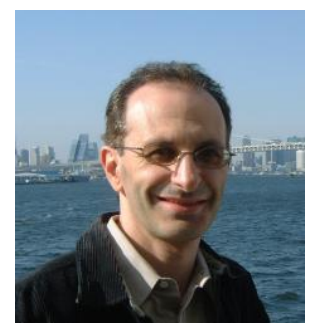

Mughees Khan is a staff scientist at the Wyss Institute at Harvard University. His background and interests are in semiconductor process development, micro/nanofabrication for applications in sub-wavelength optics, light generation, microscopy, bio-inspired optics, opto-mechanics and quantum information processing. He also has a background in active materials and smart structures. He received his B.Sc. in Mechanical Engineering (1997) from Middle East Technical University and his M.S. and Ph.D. from Texas A\&M University in Aerospace Engineering (2002) and Materials Science \&Engineering (2006). He conducted his postdoctoral work at Harvard University and has previously worked in the energy sector.

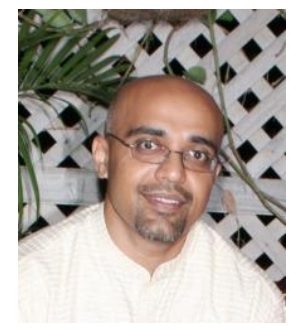


Joanna Aizenberg is the Amy Smith Berylson Professor of Materials Science, Professor of Chemistry and Chemical Biology, Radcliffe Professor and the Director of the Kavli Institute for Bionano Science and Technology at Harvard University. She received her M.S. degree with honors in chemistry in 1984 from Moscow State University, and a Ph.D. degree with honors in structural biology in 1996 at the Weizmann Institute of Science. Aizenberg joined Bell Laboratories-Lucent as a member of the technical staff in 1998, and the faculty of Harvard University in 2007. She pursues a broad range of research interests that include self-assembly, nanofabrication, biomimetics, crystal engineering, surface chemistry, and adaptive materials. Aizenberg is a fellow of the American Association for the Advancement of Science and she served on the Board on Physics and Astronomy of the National Academies and on the Board of Directors of the Materials Research Society.

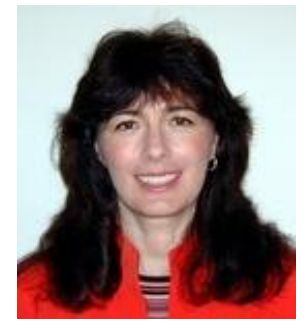

